Technical Report

MSR-TR-99-41

\title{
THE SCALING WINDOW OF THE 2-SAT TRANSITION
}

\author{
Béla Bollobás, ${ }^{2,3}$ Christian Borgs, ${ }^{1}$ Jennifer T. Chayes, ${ }^{1}$ \\ Jeong Han Kim, ${ }^{1}$ and David B. Wilson ${ }^{1}$ \\ ${ }^{1}$ Microsoft Research, Redmond, Washington \\ ${ }^{2}$ Department of Mathematical Sciences, University of Memphis \\ ${ }^{3}$ Trinity College, Cambridge, England
}

Submitted September 5, 1999; Revised January 3, 2001

\begin{abstract}
We consider the random 2-satisfiability problem, in which each instance is a formula that is the conjunction of $m$ clauses of the form $x \vee y$, chosen uniformly at random from among all 2-clauses on $n$ Boolean variables and their negations. As $m$ and $n$ tend to infinity in the ratio $m / n \rightarrow \alpha$, the problem is known to have a phase transition at $\alpha_{c}=1$, below which the probability that the formula is satisfiable tends to one and above which it tends to zero. We determine the finite-size scaling about this transition, namely the scaling of the maximal window $W(n, \delta)=\left(\alpha_{-}(n, \delta), \alpha_{+}(n, \delta)\right)$ such that the probability of satisfiability is greater than $1-\delta$ for $\alpha<\alpha_{-}$and is less than $\delta$ for $\alpha>\alpha_{+}$. We show that

$$
W(n, \delta)=\left(1-\Theta\left(n^{-1 / 3}\right), 1+\Theta\left(n^{-1 / 3}\right)\right),
$$

where the constants implicit in $\Theta$ depend on $\delta$. We also determine the rates at which the probability of satisfiability approaches one and zero at the boundaries of the window. Namely, for $m=(1+\varepsilon) n$, where $\varepsilon$ may depend on $n$ as long as $|\varepsilon|$ is sufficiently small and $|\varepsilon| n^{1 / 3}$ is sufficiently large, we show that the probability of satisfiability decays like $\exp \left(-\Theta\left(n \varepsilon^{3}\right)\right)$ above the window, and goes to one like $1-\Theta\left(n^{-1}|\varepsilon|^{-3}\right)$ below the window. We prove these results by defining an order parameter for the transition and establishing its scaling behavior in $n$ both inside and outside the window. Using this order parameter, we prove that the 2-SAT phase transition is continuous with an order parameter critical exponent of 1 . We also determine the values of two other critical exponents, showing that the exponents of 2-SAT are identical to those of the random graph.
\end{abstract}

Keywords: 2-SAT, satisfiability, constraint satisfaction problem, phase transition, finite-size scaling, critical exponents, random graph, order parameter, spine, backbone. 


\section{Introduction And Statement of Results}

There has recently been interest in a new field emerging at the intersection of statistical physics, discrete mathematics, and theoretical computer science. The field is characterized by the study of phase transitions in combinatorial structures arising in problems from theoretical computer science.

Perhaps the most interesting phenomena in statistical physics are phase transitions. These transitions occur in systems with infinitely many degrees of freedom, i.e. systems specified by infinitely many random variables. Physically, the transitions represent changes in the state of the system; mathematically, the transitions are manifested as nonanalyticities in relevant functions of an external control parameter, such as the temperature. In systems with a large but finite number of degrees of freedom, one can study the approach to nonanalytic behavior. This study is called finite-size scaling. In systems with continuous phase transitions characterized by critical exponents, the form of the finite-size scaling turns out to be related to these exponents.

Discrete mathematics often focuses on the study of large combinatorial structures. Random versions of these structures (with respect to natural distributions) are discrete systems with large but finite numbers of degrees of freedom. In the limit of an infinite number of degrees of freedom, these systems can and often do undergo phase transitions. The study of threshold phenomena emerging in these large combinatorial structures is therefore analogous to finite-size scaling in statistical physics.

The theory of complexity focuses on the difficulty of solving certain combinatorial problems which arise naturally in theoretical computer science. The complexity of a given problem is determined by the difficulty of solving any instance of the problem (i.e., in the worst case). Researchers have also studied randomly chosen instances of certain problems, and determined average- or typical-case complexity. However, even when it is determined that a problem is easy or hard on average, it is not clear what properties characterize the hard instances.

The convergence of these three disciplines is a consequence of the recent observation that one can define control parameters in terms of which certain theoretical computer science problems undergo phase transitions, and the even more interesting observation that the hardest instances of these problems seem to be concentrated at the phase transition point. The problem for which this phenomenon has been studied most extensively is the $k$-satisfiability problem. Our work is the first complete, rigorous analysis of finite-size scaling for a satisfiability problem.

The $k$-satisfiability ( $k$-SAT) problem is a canonical constraint satisfaction problem in theoretical computer science. Instances of the problem are formulae in conjunctive normal form: a $k$-SAT formula is a conjunction of $m$ clauses, each of which is a disjunction of length $k$. The $k$ elements of each clause are chosen from among $n$ Boolean variables and their negations. Given a formula, the decision version of the problem is whether there exists an assignment of the $n$ variables satisfying the formula. 
It is known that the $k$-satisfiability problem behaves very differently for $k=2$ and $k \geq 3$ Coo71]. For $k=2$, the problem is in $\mathrm{P}$ [Coo71]; indeed, it can be solved by a linear time algorithm [APT79]. For $k \geq 3$, the problem is NP-complete [Coo71, so that in the worst case it is difficult to determine whether a $k$-SAT formula is satisfiable or not - assuming $\mathrm{P} \neq \mathrm{NP}$. Note, however, that even for $k=2$, variants of the $k$-SAT problem are difficult. For example, the MAX-2-SAT problem, in which one determines whether the maximum number of satisfiable clauses in a 2-SAT formula is bounded by a given integer, is an NP-complete problem GJS76 (see also GJ79]), and even approximating it to within a factor of $4 / 3-\varepsilon$ is NP-hard Hås97.

More recently, it has been realized that-rather than focusing on worst-case instancesit is often useful to study typical instances of the fixed- $k$ problem as a function of the parameter $\alpha=m / n$. Consider the random $k$-SAT problem, in which formulae are generated by choosing uniformly at random from among all possible clauses. As $m$ and $n$ tend to infinity with limiting ratio $m / n \rightarrow \alpha$, considerable empirical evidence suggests that the random $k$-SAT problem undergoes a phase transition at some value $\alpha_{c}(k)$ of the parameter $\alpha$ (MSL92, [CA93, [LT93], KS94): For $\alpha<\alpha_{c}$, a random formula is satisfiable with probability tending to one as $m$ and $n$ tend to infinity in the fixed ratio $\alpha=m / n$, while if $\alpha>\alpha_{c}$, a random formula is unsatisfiable with probability tending to one as $m$ and $n$ tend to infinity, again with $m / n \rightarrow \alpha$.

Existence of the phase transition is on a different footing for $k=2$ and $k \geq 3$. For $k=2$, it was shown by Goerdt (Goe92, Goe96]), Chvátal and Reed [CR92], and Fernandez de la Vega [Fer92] that a transition occurs at $\alpha_{c}(2)=1$. For $k \geq 3$, it may not be possible to locate the exact value of the transition point. However, there has been considerable work bounding the value of the presumed 3-SAT threshold from below and above. Using a succession of increasingly sophisticated and clever algorithms for finding SAT solutions with high probability, lower bounds on $\alpha_{c}(3)$ were improved from 1 ( CF86, [CF90, [CR92]) to 1.63 [BFU93 to 3.003 [ES96] to 3.145 [Ach00] to 3.26 AS00. Bounding the probability of finding a solution by the expected number of solutions gave an upper bound on $\alpha_{c}(3)$ of 5.191 [FP83]; increasingly sophisticated counting arguments gave a succession of improved upper bounds on $\alpha_{c}(3)$ from 5.08 [EF95 to 4.758 [KMPS95 to 4.643 [DB97] to 4.602 [KKK96 to 4.596 [JSV00]. More recently a bound of 4.506 [DBM99] has been announced. Although these bounds are relatively tight, they nevertheless allow for the possibility of a non-sharp transition. However, motivated by the empirical evidence, Friedgut and later Bourgain showed that indeed there is a sharp transition [FB99 (although they did not prove that the probability of satisfiability approaches a limit). These proofs were based on a general argument which shows that global, as opposed to local, phenomena lead to sharp transitions. However, the existence of a limiting threshold is still an open problem.

Having established the sharpness of the transition, the next step is to analyze some of its properties. Finite-size scaling is the study of changes in the transition behavior due to finite-size effects, in particular, broadening of the transition region for finite $n$. To be precise, for $0<\delta<1$, let $\alpha_{-}(n, \delta)$ be the supremum over $\alpha$ such that for $m=\alpha n$, the 
probability of a random formula being satisfiable is at least $1-\delta$. Similarly, let $\alpha_{+}(n, \delta)$ be the infimum over $\alpha$ such that for $m=\alpha n$, the probability of a random formula being satisfiable is at most $\delta$. Then, for $\alpha$ within the scaling window

$$
W(n, \delta)=\left(\alpha_{-}(n, \delta), \alpha_{+}(n, \delta)\right),
$$

the probability of a random formula being satisfiable is between $\delta$ and $1-\delta$. Since, by [FB99], for all $\delta,\left|\alpha_{+}(n, \delta)-\alpha_{-}(n, \delta)\right| \rightarrow 0$ as $n \rightarrow \infty$, we say that the scaling window represents the broadening of the transition due to finite-size effects. Sometimes we shall omit the explicit $\delta$ dependence of $\alpha_{ \pm}(n, \delta)$ and $W(n, \delta)$, writing instead $\alpha_{ \pm}(n)$ and $W(n)$. In these cases, the power laws we quote will be uniform in $\delta$, but the implicit constants may depend on $\delta$.

The first model for which such broadening was established rigorously is the random graph model. The phase transition for this model, namely the sudden emergence of a giant component, was already proved by Erdős and Rényi ([ER60], [ER61]). But the characteristic width of the transition was (correctly) investigated only 24 years later by Bollobás [Bol84] (see also [Bol85] and the references therein). In particular, this work showed that the width of the scaling window $W(n)$ is $n^{-1 / 3+o(1)}$; the precise growth rate was later shown to be $\Theta\left(n^{-1 / 3}\right)$ by Łuczak Euc90. Many additional properties of the phase transition were then determined using generating functions EPW94. [JKŁP94. For the finite-dimensional analogue of the random graph problem, namely percolation on a low-dimensional hypercubic lattice, the broadening was established by Borgs, Chayes, Kesten and Spencer ([BCKS98a], BCKS98b]), who also related the power law form of $\alpha_{ \pm}(n)$ to the critical exponents of the percolation model.

The question of finite-size scaling in the $k$-SAT model was first addressed by Kirkpatrick and Selman [KS94], who presented both a heuristic framework and empirical evidence for analysis of the problem. There has also been subsequent empirical ([SK96], [MZKST99]) and theoretical ([MZ96], [MZ97], [MZKST99]) work, the latter using the replica method familiar from the study of disordered, frustrated models in condensed matter physics (see [MPV87 and references therein). Although the theoretical work has yielded a good deal of insight, the empirical work on finite-size scaling has been misleading Wil00, and rigorous progress on finite-size scaling in $k$-SAT has been quite limited.

In this work, we address the question of finite-size scaling in the 2-SAT problem; in particular, we obtain the power law form of the scaling window $W(n)=\left(\alpha_{-}(n), \alpha_{+}(n)\right)$, together with the rates of convergence at the boundaries of the window. Previous work on 2-SAT by Goerdt Goe99 has shown that $\alpha_{-}(n) \geq 1-O(1 / \sqrt{\log n})$, while Verhoeven Ver99 has recently obtained the result $\alpha_{+}(n) \leq 1+O\left(n^{-1 / 4}\right)$. Numerical work on the scaling window for 2-SAT is somewhat controversial: While earlier simulations [MZKST99] suggested that the window scales like $W(n)=\left(1-\Theta\left(n^{-1 / 2.8}\right), 1+\Theta\left(n^{-1 / 2.8}\right)\right)$, recent simulations by Wilson [Wi198] indicate that the 2-SAT formulae considered in [MZKST99] are not long enough to reach the asymptotic regime.' Indeed, we shall prove in this paper

\footnotetext{
${ }^{1}$ As usual, $f=\Theta(g)$ means that there exist positive, finite constants $c_{1}$ and $c_{2}$ such that $c_{1} \leq f / g \leq c_{2}$. Unless noted otherwise, these constants are universal. In fact, in the above formulae for $W(n)$, the constants depend on $\delta$.
} 
that $W(n)=\left(1-\Theta\left(n^{-1 / 3}\right), 1+\Theta\left(n^{-1 / 3}\right)\right)$, as conjectured earlier by Bollobás, Borgs, Chayes and Kim BBCK98 and predicted numerically in Wil98. We also show how the probability of satisfiability tends to 1 and 0 at the edges of the window.

In order to state our results precisely, we need a little notation. Let $x_{1}, \ldots, x_{n}$ denote $n$ Boolean variables. Writing $\bar{x}$ for the negation of $x$, our $n$ variables give $2 n$ literals $x_{1}, \ldots, x_{n}, \bar{x}_{1}, \ldots, \bar{x}_{n}$. Two literals $x$ and $y$ are said to be strictly distinct if neither $x=y$ nor $x=\bar{y}$. A $k$-clause is a disjunction $C=u_{1} \vee \cdots \vee u_{k}$ of $k$ strictly distinct literals, and a $k$-SAT formula is a conjunction $F=C_{1} \wedge \cdots \wedge C_{m}$ of $k$-clauses $C_{1}, \ldots, C_{m}$. We say that $H$ is a subformula of $F$ if it can be obtained from $F$ by deleting some of its clauses. A $k$-SAT formula $F=F\left(x_{1}, \ldots, x_{n}\right)$ is said to be satisfiable, or SAT, if there exists a truth assignment $\eta_{i} \in\{0,1\}, i=1, \ldots, n$, such that $F\left(\eta_{1}, \ldots, \eta_{n}\right)=1$. Here, as usual, 0 stands for the logical value FALSE, and 1 is the logical value TRUE. We write " $F$ is SAT" if the formula $F$ is satisfiable, and " $F$ is UNSAT" if the formula $F$ is not satisfiable. We also sometimes use the alternative notation $\operatorname{SAT}(F)$ and $\operatorname{UNSAT}(F)$ to denote these two cases.

We consider the random 2-SAT problem in two essentially equivalent forms, given by $a$ priori different probability distributions of random 2-SAT formulae on $x_{1}, \ldots, x_{n}$. First, we consider the probability space of formulae $F_{n, m}$ chosen uniformly at random from all 2-SAT formulae with exactly $m$ different clauses. (Here $x \vee y$ is considered to be the same as $y \vee x$, but different from e.g. $x \vee \bar{y}$.) Second, we consider the space of formulae $F_{n, p}$ with 2-clauses on $x_{1}, \ldots, x_{n}$ chosen independently with probability $p$. In this introduction, we shall state theorems in terms of the $F_{n, m}$; the equivalent theorems for the $F_{n, p}$ will be given in Section 3. The conversion between the two formulations of the problem is given in Appendix A. In both cases, we use $\mathbb{P}(A)$ to denote the probability of an event $A$.

As usual in 2-SAT, it is convenient to study the phase transition in terms of the parameter $\varepsilon$ representing the deviation of $\alpha$ from its critical value:

$$
m=(1+\varepsilon) n \text {. }
$$

When studying finite-size effects, we shall take the parameter $\varepsilon$ to depend on $n$. Our analysis shows that the appropriate scaling of $\varepsilon$ is $n^{-1 / 3}$, so that it is natural to define yet another parameter $\lambda=\lambda_{n}$ according to

$$
\varepsilon=\lambda_{n} n^{-1 / 3}
$$

and distinguish the cases $\lambda_{n}$ bounded, $\lambda_{n} \rightarrow \infty$ and $\lambda_{n} \rightarrow-\infty$.

Our main result is the following theorem.

Theorem 1.1. There are constants $\varepsilon_{0}$ and $\lambda_{0}, 0<\varepsilon_{0}<1,0<\lambda_{0}<\infty$, such that

$$
\mathbb{P}\left(F_{n, m} \text { is SAT }\right)= \begin{cases}1-\Theta\left(\frac{1}{\left|\lambda_{n}\right|^{3}}\right) & \text { if }-\varepsilon_{0} n^{1 / 3} \leq \lambda_{n} \leq-\lambda_{0}, \\ \Theta(1) & \text { if }-\lambda_{0} \leq \lambda_{n} \leq \lambda_{0}, \\ \exp \left(-\Theta\left(\lambda_{n}^{3}\right)\right) & \text { if } \lambda_{0} \leq \lambda_{n} \leq \varepsilon_{0} n^{1 / 3} .\end{cases}
$$


Note that the behaviors for $\lambda_{n}<0$ and $\lambda_{n}>0$ can be cast in the same form by writing $\mathbb{P}\left(F_{n, m}\right.$ is $\left.\mathrm{SAT}\right)=1-\Theta\left(\left|\lambda_{n}\right|^{-3}\right)=\exp \left(-\Theta\left(\left|\lambda_{n}\right|^{-3}\right)\right)$.

Theorem 1.1 gives us the exact form of the scaling window:

Corollary 1.2. For all sufficiently small $\delta>0$, the scaling window (1.1) is of the form

$$
W(n, \delta)=\left(1-\Theta\left(n^{-1 / 3}\right), 1+\Theta\left(n^{-1 / 3}\right)\right),
$$

where the constants implicit in the definition of $\Theta$ depend on $\delta$, and are easily calculated from equation (1.4).

Of course, the theorem gives us more than the boundaries of the window; it also gives us the rates of approach of the probability of satisfiability at these boundaries. As an easy special case of the rate result at the upper boundary, note that if $\varepsilon$ is positive and independent of $n$, then our result for $\lambda>\lambda_{0}$ gives that

$$
\mathbb{P}\left(F_{n, m} \text { is } \mathrm{SAT}\right)=\exp \left(-\Theta\left(\varepsilon^{3} n\right)\right) .
$$

This strengthens both the result of Fernandez de la Vega [Fer98 that $\mathbb{P}\left(F_{n, m}\right.$ is SAT $)=$ $O(\exp (-f(\varepsilon) \sqrt{n}))$ and the recent improvement of Achlioptas and Molloy AM98 that $\mathbb{P}\left(F_{n, m}\right.$ is SAT $)=O(\exp (-f(\varepsilon) n))$ for some $f(\varepsilon)>0$.

We remark that in the random graph model, the existence of a complex component, i.e. a connected component with more than one cycle, is roughly analogous to the existence of a contradiction in a random 2-SAT formula. When there are $m=\frac{1}{2} n\left(1+\lambda n^{-1 / 3}\right)$ edges, Britikov [Bri89] showed

$$
\mathbb{P}\left(\begin{array}{l}
G_{n, m} \text { contains no } \\
\text { complex component }
\end{array}\right)=\left(1+o_{n}(1)\right) \begin{cases}1-\frac{5+o_{\lambda}(1)}{24} \frac{1}{|\lambda|^{3}} & \text { if }-\omega(n) \leq \lambda \leq-\lambda_{0}, \\
P(\lambda) & \text { if }-\lambda_{0} \leq \lambda \leq \lambda_{0}, \\
\frac{\sqrt{2 \pi}+o_{\lambda}(1)}{2^{1 / 4} \Gamma(1 / 4)} \frac{e^{-\lambda^{3} / 6}}{\lambda^{3 / 4}} & \text { if } \lambda_{0} \leq \lambda \leq \omega(n),\end{cases}
$$

where $P(\lambda)$ is an explicit power series in $\lambda$, and $\omega(n)$ is an unspecified slowly growing function of $n$. Later this formula was shown to be valid for $|\lambda| \ll n^{1 / 12}$ [JKŁP94, pg. 289]. Observe that in comparison, the analogous bounds for random 2-SAT in Theorem 1.1 are not so precise, but they hold for the full range of $|\lambda| \ll n^{1 / 3}$.

The key to our analysis is the introduction of an order parameter for the 2-SAT phase transition. As usual in statistical physics, an order parameter is a function which vanishes on one side of the transition and becomes non-zero on the other side. Control of the growth of the order parameter was the key to Bollobás' analysis of finite-size scaling in the random graph [Bol84], and to Borgs, Chayes, Kesten and Spencer's analysis of finite-size scaling in percolation $B C K S 98 b$. Our order parameter for satisfiability is the average density of the spine of a Boolean formula, which we define as follows. Given a formula $F$ in conjunctive normal form, we define the spine $S(F)$ as the set of literals $x$ such that there is a satisfiable subformula $H$ of $F$ for which $H \wedge x$ is not satisfiable,

$$
S(F)=\{x \mid \exists H \subset F, H \text { is SAT and } H \wedge x \text { is } \mathrm{UNSAT}\} .
$$


Our notion of the spine was motivated by the insightful concept of the backbone, $B(F)$, introduced by Monasson and Zecchina MZ96 - where the backbone density $|B(F)| / n$ was originally called "the fraction of frozen variables." The backbone $B(F)$ is the set of literals that are required to be FALSE in any assignment that minimizes the number of unsatisfied clauses in $F$. It is easy to see that $B(F) \subset S(F)$, and in particular $B(F)=S(F)$ if $F$ is satisfiable. One of the principal differences between the spine and the backbone is that the spine is monotone in the sense that adding clauses to a formula only enlarges its spine. It is the monotonicity which enables us to achieve analytical control of the spine. In addition, we have found that the spine is easier to simulate than the backbone [Wil98]. We believe that the spine will become an important tool in the analysis of satisfiability problems.

Consider now a satisfiable 2-SAT formula $F$. It is not hard to see that the addition of the 2-clause $C=x \vee y$ makes $F$ (or, more precisely, makes $F \wedge C$ ) unsatisfiable if and only if both $x$ and $y$ lie in the spine. Building a random 2-SAT formula by adding clauses one by one at random to an initially empty (and hence satisfiable) formula, we can therefore control the probability that a formula is satisfiable if we have sufficient control of the spine in each step. This is the strategy we shall follow to prove Theorem 1.1.

In the course of proving Theorem 1.1, we obtain detailed estimates on the expectation and variance of the size of the spine inside the scaling window $m \in\left[n-\Theta\left(n^{2 / 3}\right), n+\right.$ $\left.\Theta\left(n^{2 / 3}\right)\right]$, i.e. the finite-size scaling of the spine. Before stating these results, however, let us give the behavior of the size of $S\left(F_{n, m}\right)$ on the scale $n$. To this end, let $\vartheta:(0, \infty) \rightarrow(0,1)$ be the function satisfying

$$
1-\vartheta(\varepsilon)=\exp [-(1+\varepsilon) \vartheta(\varepsilon)]
$$

i.e.

$$
\vartheta(\varepsilon)=1-\sum_{k=1}^{\infty} \frac{k^{k-1}}{k !}(1+\varepsilon)^{k-1} e^{-(1+\varepsilon) k} .
$$

The $k$ th term in (1.9) is the probability that a Poisson birth-and-death process with birth rate $1+\varepsilon$ will have size $k$, while $\vartheta(\varepsilon)$ is the probability that it is infinite. Note that $\vartheta(\varepsilon)=2 \varepsilon+O\left(\varepsilon^{2}\right)$ for positive $\varepsilon$ sufficiently small. The size of the spine is given by:

Theorem 1.3. For any fixed $\varepsilon \in\left(-\varepsilon_{0}, \varepsilon_{0}\right)$, where $\varepsilon_{0}$ is the constant from Theorem 1.1, we have

$$
E\left(\left|S\left(F_{n, m}\right)\right|\right)= \begin{cases}\Theta\left(\varepsilon^{-2}\right) & \text { if } \varepsilon<0 \\ \Theta\left(n^{2 / 3}\right) & \text { if } \varepsilon=0 \\ 2 n \vartheta(\varepsilon)+o(n) & \text { if } \varepsilon>0 .\end{cases}
$$

The behavior above, coupled with the role of the spine in the proof of Theorem 1.1, justifies our identification of the density of the spine as an order parameter for the 2SAT transition. In the language of phase transitions, Theorem [1.3 implies that the 
2-SAT transition is second-order (or continuous), with order parameter critical exponent $\beta=1$. Here, as usual, we say that the order parameter has critical exponent $\beta$ if $\lim _{n \rightarrow \infty} E\left(\left|S\left(F_{n, m}\right)\right|\right) / n=\Theta\left(\varepsilon^{\beta}\right)$ as $\varepsilon \downarrow 0$, see discussion following Remark 1.5.

The next theorem states our results for the finite-size scaling of the spine $S\left(F_{n, m}\right)$ :

Theorem 1.4. Let $\varepsilon_{0}$ and $\lambda_{0}$ be the constants in Theorem 1.1. Suppose $\left|\lambda_{n}\right| \leq \varepsilon_{0} n^{1 / 3}$. Then

$$
E\left(\left|S\left(F_{n, m}\right)\right|\right)= \begin{cases}\frac{1}{2} \lambda_{n}^{-2} n^{2 / 3}(1+o(1)) & \text { if } \lambda_{n}<-\lambda_{0} \\ \Theta\left(n^{2 / 3}\right) & \text { if }\left|\lambda_{n}\right| \leq \lambda_{0} \\ 4 \lambda_{n} n^{2 / 3}(1+o(1)) & \text { if } \lambda_{n}>\lambda_{0},\end{cases}
$$

where the o(1) terms represent errors which go to zero as $\left|\lambda_{n}\right| \rightarrow \infty$ and $\varepsilon=\lambda_{n} n^{-1 / 3} \rightarrow 0$.

Remark 1.5. In the course of proving Theorems 1.1, 1.3 and 1.4, we shall prove bounds on the variance of $\left|S\left(F_{n, m}\right)\right|$ which allow us to generalize the above statements in expectation to statements in probability.

Statistical mechanical models with second-order (i.e., continuous) transitions are often characterized by critical exponents which describe the behavior of fundamental quantities at or approaching the critical point. It turns out (see [BCKS98B and announcements in Cha98 and [CPS99) that it is possible to read off some of these exponents from the finite-size scaling form of the order parameter and the scaling window. In particular, the scaling of the order parameter at the critical point allows us to evaluate the so-called field exponent $\delta$ as

$$
E\left(\left|S\left(F_{n, m}\right)\right|\right)=\Theta\left(n^{\frac{\delta}{1+\delta}}\right) \quad \text { if } \quad\left|\lambda_{n}\right|<\lambda_{0} .
$$

Similarly (again using [BCKS98b], [Cha98 and [CPS99]), the scaling of the window allows us to identify the exponent sum $2 \beta+\gamma$, according to

$$
W(n, \delta)=\left(1-\Theta\left(n^{-1 /(2 \beta+\gamma)}\right), 1+\Theta\left(n^{-1 /(2 \beta+\gamma)}\right)\right),
$$

where $\beta$ is the order parameter exponent described above, i.e.

$$
\lim _{n \rightarrow \infty} \frac{1}{n} E\left(\left|S\left(F_{n, m}\right)\right|\right)=\Theta\left(\varepsilon^{\beta}\right) \quad \text { as } \quad \varepsilon \downarrow 0,
$$

and $\gamma$ is the so-called susceptibility exponent. Comparing equations (1.14), (1.13), and (1.12) to Theorem 1.3, Corollary 1.2, and Theorem 1.4, respectively, we get the following.

Corollary 1.6. The 2-SAT transition is a second-order (i.e. continuous) transition with critical exponents:

$$
\beta=1, \quad \gamma=1, \quad \text { and } \quad \delta=2 .
$$

Thus we have proved that the critical exponents of the random 2-SAT problem are identical to those of the random graph. See [BBCKW00] for a more detailed discussion of the critical exponents for 2-SAT. 
The organization of this paper is as follows. In Section 2, we discuss the well-known representation of 2-SAT formulae as directed graphs, a representation we use extensively in our proofs. In that section, we also derive new results on various representations of the spine in terms of directed graphs. While most of the results in Section 8 concern given formulae, not distributions of formulae, a final result there gives a mapping of a distribution of certain sets in the graphical representation of random 2-SAT into the standard random graph model. In Section 3, we state our main technical estimates on the expectation and variance of the size of the spine, and formulate an analogue of Theorem 1.1 for the distribution $F_{n, p}$. We then outline the strategy of our proof, giving first our heuristic for the expected size of the spine, and then showing how this will be used to obtain the size of the scaling window (Theorem 1.1). While the width of the scaling window can be determined from the spine expectation and variance estimates alone, the rate of approach from above in Theorem 1.1 requires that a sufficiently large spine forms with extremely high probability. In order to prove this, in Section 4 , we define structures we call "hourglasses" which are basically precursors to the spine, and we state a theorem giving conditions under which a giant hourglass forms. The proof of the hourglass theorem is given in Section 9. In Section 14, we use the expectation and variance results on the spine, and the hourglass theorem, to establish the analogue of Theorem 1.1 for the distribution $F_{n, p}$. In Sections 5 and 6, we develop some machinery from random graph theory and derive moment bounds, which enable us to prove the expected size and variance results for the spine in Sections 7 and 8, respectively. Appendix A contains the conversion from our results on $F_{n, p}$ to $F_{n, m}$, and Appendix B establishes a technical result on the cluster size distribution in the random graph problem.

\section{The Digraph Representation of 2-SAT}

In the digraph representation, each 2-SAT formula corresponds to a certain directed graph (or digraph) $D_{F}$. To motivate the mapping of $F$ into $D_{F}$, note that $F$ is satisfiable if and only if all clauses in $F$ are satisfiable. Thus, if $F$ contains a clause $C=x \vee y$, a satisfying truth assignment with $x$ set to FALSE requires that $y$ is set to TRUE, and a satisfying assignment with $y$ set to FALSE requires that $x$ is set to TRUE. So the clause $x \vee y$ corresponds to the logical implications $\bar{x}=$ TRUE $\Longrightarrow y=$ TRUE and $\bar{y}=$ TRUE $\Longrightarrow x=$ TRUE. We shall encode this fact in the digraph $D_{F}$ by including the edges $\bar{x} \rightarrow y$ and $\bar{y} \rightarrow x$ in $D_{F}$ iff $F$ contains a clause $C=x \vee y$.

To be precise, given a 2-SAT formula $F$, define the digraph $D_{F}$ as the directed graph with vertex set²

$$
[n]=\left\{x_{1}, \ldots, x_{n}, \bar{x}_{1}, \ldots, \bar{x}_{n}\right\}
$$

and edge set

$$
E_{F}=\{x \rightarrow y \mid(\bar{x} \vee y) \text { is a clause in } F\} .
$$

\footnotetext{
${ }^{2}$ Note that we deviate from the standard notation, where $[n]$ stands for the set $\{1,2, \ldots, n\}$.
} 
Since $(x \vee y)$ and $(y \vee x)$ are considered to be the same clause, the digraph $D_{F}$ contains the edge $\bar{x} \rightarrow y$ if and only if it contains the edge $\bar{y} \rightarrow x$. As usual, an oriented path in $D_{F}$ is a sequence of vertices $v_{0}, v_{1}, \ldots, v_{k} \in[n]$ and edges $v_{i} \rightarrow v_{i+1}$ for $i=0,1, \ldots, k-1$. We say that this path is a path from $x$ to $y$ if $v_{0}=x$ and $v_{k}=y$. We write $x \rightsquigarrow y$, or sometimes simply $x \rightsquigarrow y$, if $D_{F}$ contains an oriented path from $x$ to $y$. By convention, we shall say $x \rightsquigarrow x$ for all $x$. Finally, we say that $D_{F}$ contains a contradictory cycle if $x \rightsquigarrow \bar{x}$ and $\bar{x} \rightsquigarrow x$ for some $x \in[n]$.

The following lemma connecting the structure of the digraph $D_{F}$ with the satisfiability of the formula $F$ is implicit in all digraph analyses of 2-SAT, see e.g. [Goe92. For completeness, we shall give an explicit proof here.

Lemma 2.1. A 2-SAT formula $F$ is satisfiable if and only if the digraph $D_{F}$ has no contradictory cycle.

Proof. Let us first assume that $F$ is satisfiable, with satisfying assignment $\eta_{i} \in\{0,1\}, i=$ $1, \ldots, n$. Consider an edge $\bar{x} \rightarrow y$ in the corresponding digraph. Since $F\left(\eta_{1}, \ldots, \eta_{n}\right)=1$, the presence of the edge $\bar{x} \rightarrow y$ gives the logical implication $x=$ FALSE $\Longrightarrow y=$ TRUE. A contradictory cycle $x \rightsquigarrow \bar{x} \rightsquigarrow x$ therefore gives the logical implication $x=$ TRUE $\Longrightarrow$ $x=$ FALSE $\Longrightarrow x=$ TRUE, which is not compatible with any truth assignment for $x$.

We prove the converse by induction on the number $n$ of variables. For $n=1$ there is nothing to prove. Turning to the induction step, suppose that the digraph $D_{F}$ has no contradictory cycles. We claim that in this case $F$ is satisfiable. To this end, we first recall the definition of strongly connected components for directed graphs. We say that two vertices $x$ and $y$ in a directed graph are strongly connected if $x \rightsquigarrow y \rightsquigarrow x$, i.e. if the directed graph $D_{F}$ has a cycle containing $x$ and $y$. The strongly connected component of a vertex $x$ is the induced subgraph of $D_{F}$ containing the set of vertices

$$
C_{S}(x)=\{y \mid x \rightsquigarrow y \rightsquigarrow x\} .
$$

Somewhat loosely, we call $C_{S}(x)$ the strong component of $x$. Clearly, the strong component partitions the vertex set $[n]$. We define a partial order $\leq$ on the set of all strong components by taking $C_{S}(x) \leq C_{S}(y)$ if $x \rightsquigarrow y$, and so $x^{\prime} \rightsquigarrow y^{\prime}$ for all $x^{\prime} \in C_{S}(x)$ and $y^{\prime} \in C_{S}(y)$. Let $C_{S}$ be a minimal element in this partial order, i.e. let $C_{S}$ be a strong component such that $D_{F}$ contains no edge $x \rightarrow y$ with $x \notin C_{S}$ and $y \in C_{S}$. For a set of literals $M$, let

$$
\bar{M}=\{y \mid \bar{y} \in M\} .
$$

Since $D_{F}$ has no contradictory cycle, $C_{S} \cap \overline{C_{S}}=\emptyset$. Furthermore, since $C_{S}$ is a minimal element in our partial order, $\overline{C_{S}}$ must be a maximal element. If we set all literals in $C_{S}$ to FALSE, and so all literals in $\overline{C_{S}}$ to TRUE, then all clauses in $F$ containing at least one literal from $C_{S} \cup \overline{C_{S}}$ are TRUE. This process removes all the variables corresponding to literals in $C_{S}$ and $\overline{C_{S}}$ from $[n]$, and all clauses involving these variables from $F$, leading to a new 2-SAT formula $F^{\prime}$. Since the graph $D_{F^{\prime}}$ is a subgraph of $D_{F}$, it contains no contradictory cycles either. Using the inductive hypothesis, we obtain a satisfying 
assignment for $F$, which completes the proof of the converse and hence of the theorem.

Remark 2.2. If $F$ is a mixture of one and two-clauses, i.e. if it is of the form $F=H \wedge$ $x_{1} \wedge \cdots \wedge x_{k}$ where $H$ is a 2-SAT formula and $x_{1}, \ldots, x_{k}$ are literals, we define $D_{F}$ by including the edges $\bar{x}_{i} \rightarrow x_{i}, i=1, \ldots, k$, in addition to the edges in $D_{H}$. It is not hard to see that the above proof applies also to this situation, giving again that $F$ is SAT if and only if $D_{F}$ contains no contradictory cycles.

While the previous lemma says that contradictions in a formula correspond to cycles in the digraph, the next lemma says that the spine of a formula corresponds to "half-cycles" in the digraph. This graphical description of the spine is central to our analysis.

Lemma 2.3. For every 2-SAT formula $F$,

$$
S(F)=\left\{x \mid \underset{D_{F}}{x \rightsquigarrow \bar{x}}\right\}
$$

where $D_{F}$ is the digraph corresponding to $F$.

Proof. Suppose that $x \rightsquigarrow \bar{x}$, and let $x=v_{0} \rightarrow v_{1} \rightarrow \cdots \rightarrow v_{r-1} \rightarrow v_{r}=\bar{x}$ be a shortest directed path from $x$ to $\bar{x}$ in $D_{F}$. Then no literal appears twice in the path, although a literal and its negation may well do so. Let $\ell$ be the smallest positive integer such that $v_{\ell}$ is not strictly distinct from all of $v_{0}, v_{1}, \ldots, v_{\ell-1}$, and let $0 \leq k<\ell$ be such that $v_{\ell}=\bar{v}_{k}$. Then $H=\left(\bar{v}_{0} \vee v_{1}\right) \wedge\left(\bar{v}_{1} \vee v_{2}\right) \wedge \cdots \wedge\left(\bar{v}_{\ell-1} \vee v_{\ell}\right)$ is a subformula of $F$ that is satisfied by setting each of $v_{0}, v_{1}, \ldots, v_{\ell-1}$ to FALSE. On the other hand, $H \wedge x=H \wedge v_{0}$ is UNSAT since in order to satisfy it, we would have to set $v_{0}$ to TRUE, then $v_{1}$ to TRUE, and so on, ending with the requirement that $v_{\ell}$ be set TRUE. However, as $v_{k}$ is TRUE, $v_{\ell}$ is already set FALSE. This completes the proof that $x \rightsquigarrow \bar{x}$ implies that $x \in S(F)$.

Conversely, suppose that $H \subset F$ is SAT and $H^{\prime}=H \wedge x=H \wedge(x \vee x)$ is UNSAT. Then $D_{H^{\prime}}$ has a contradictory cycle $C=u \rightsquigarrow \bar{u} \rightsquigarrow u$. Since $D_{H}$ does not have a contradictory cycle, the cycle $C$ of $H^{\prime}$ contains the oriented edge $\bar{x} \rightarrow x$, say $u \rightsquigarrow \bar{u} \rightsquigarrow \bar{x} \rightarrow x \rightsquigarrow u$. But then in $D_{H}$ we have $x \rightsquigarrow u \rightsquigarrow \bar{u} \rightsquigarrow \bar{x}$, so $x \rightsquigarrow \bar{x}$. Hence if $x \in S(F)$ then $\frac{x \rightsquigarrow \bar{x}}{D_{F}}$.

Our next lemma gives an alternative representation for the spine of a 2-SAT formula $F$. In order to state it, we introduce the out-graph $D_{F}^{+}(x)$ of a vertex $x$ in $D_{F}$ as the set of vertices and edges that can be reached from $x . D_{F}^{+}(x)$ therefore has the vertex set

$$
L^{+}(x)=L_{F}^{+}(x)=\left\{y \mid \underset{D_{F}}{x \rightsquigarrow y}\right\}
$$

and contains all edges $y \rightarrow z$ in $D_{F}$ such that $y \in L_{F}^{+}(x)$. For future reference, we also introduce the in-set

$$
L^{-}(x)=L_{F}^{-}(x)=\left\{y \mid \underset{D_{F}}{y \leadsto x}\right\}
$$

and the corresponding in-graph $D_{F}^{-}(x)$. Note that $x \in L_{F}^{ \pm}(x)$ since, by our convention, $x \rightsquigarrow x$ for all $x$. 
As we shall see, the spine of a 2-SAT formula $F$ can equivalently be described as the set of literals $x$ such that $L_{F}^{+}(x)$ is not strictly distinct, where for simplicity, we say that a set $M \subset[n]$ is strictly distinct (s.d.) if the literals in $M$ are pairwise strictly distinct.

Lemma 2.4. For every 2-SAT formula $F$

$$
\begin{aligned}
\left\{x \mid x \underset{D_{F}}{x} \bar{x}\right\} & =\left\{x \mid L_{F}^{+}(x) \text { is not s.d. }\right\} \\
& =\left\{x \mid L_{F}^{+}(x) \backslash\{x, \bar{x}\} \text { is not s.d. }\right\} .
\end{aligned}
$$

Proof. We start with the first equality in (2.8). If $x \rightsquigarrow \bar{x}$, then $\{x, \bar{x}\} \subset L_{F}^{+}(x)$, so $L_{F}^{+}(x)$ is not strictly distinct. If $L_{F}^{+}(x)$ is not strictly distinct, then $\{y, \bar{y}\} \subset L_{F}^{+}(x)$ for some literal $y \in[n]$, and hence $x \rightsquigarrow y$ and $x \rightsquigarrow \bar{y}$. But $x \rightsquigarrow \bar{y}$ implies that $y \rightsquigarrow \bar{x}$, which together with $x \rightsquigarrow y$ implies $x \rightsquigarrow \bar{x}$.

To prove the second equality, we first note that the set of literals $x$ for which $L_{F}^{+}(x) \backslash$ $\{x, \bar{x}\}$ is not strictly distinct is obviously a subset of the set of literals $x$ such that $L_{F}^{+}(x)$ is not strictly distinct. We are thus left with the proof that the statement that $L_{F}^{+}(x)$ is not strictly distinct implies the (apparently stronger) statement that $L_{F}^{+}(x) \backslash\{x, \bar{x}\}$ is not strictly distinct. So let us assume that $L_{F}^{+}(x)$ is not strictly distinct. By the first equality in (2.8), this implies $x \rightsquigarrow \bar{x}$. Since the digraph of a 2-SAT formula does not contain any direct edges from $x$ to $\bar{x}$, we conclude that there must be a literal $y$ strictly distinct from $x$ such that $x \rightsquigarrow y \rightsquigarrow \bar{x}$. The latter statement implies that both $x \rightsquigarrow y$ and $x \rightsquigarrow \bar{y}$, so that $L_{F}^{+}(x) \backslash\{x, \bar{x}\}$ is not strictly distinct.

Remark 2.5. As the above proof shows, the first equality in (2.8) is true for mixed formulas of 1- and 2-SAT clauses as well. The second is obviously false for mixed formulas of 1and 2-SAT clauses, as the simple example of the formula $F=\bar{x}$ shows.

The Trimmed OUt-Graph. We end this section with a construction of a trimmed version of the out-graph $D_{F}^{+}(x)$, which we denote by $\widetilde{D}_{F}^{+}(x)$ with vertex set denoted by $\widetilde{L}_{F}^{+}(x)$. The utility of this trimmed graph is that, by projecting it to an unoriented graph, we shall be able to relate it to the more familiar random graph. Given any digraph on a subset of $\left\{x_{1}, \ldots, x_{n}, \bar{x}_{1}, \ldots, \bar{x}_{n}\right\}$ we can project it to an unoriented graph on a subset of $\left\{x_{1}, \ldots, x_{n}\right\}$ by dropping negations. In particular, each literal $x \in\left\{x_{1}, \ldots, x_{n}, \bar{x}_{1}, \ldots, \bar{x}_{n}\right\}$ gets mapped to its corresponding variable $\langle x\rangle \in\left\{x_{1}, \ldots, x_{n}\right\}$, and each clause $x \vee y$ gets mapped to the edge $\{\langle x\rangle,\langle y\rangle\}$. We call this the unoriented projection of the digraph. Specifically, for $F=F_{n, p}$, we shall compare the distribution of $\widetilde{D}_{F}^{+}(x)$ for a fixed vertex $x$ to that of the connected component of a given vertex in the random graph $G_{n, 2 p-p^{2}}$, where, as usual, $G_{n, \widetilde{p}}$ denotes the random graph on $\left\{x_{1}, \ldots, x_{n}\right\}$ that is obtained from the complete graph on $\left\{x_{1}, \ldots, x_{n}\right\}$ by keeping each edge with probability $\widetilde{p}$. We use the symbol $C_{n, \widetilde{p}}(x)$ to denote the connected component of the vertex $\langle x\rangle$ in $G_{n, \widetilde{p}}$.

Construction of the trimmed out-graph. 
We construct the trimmed out-graph $\widetilde{D}_{F}^{+}(x)$ by doing a local search in $D_{F}$ starting from literal $x$, and at the same time we construct that portion of the random graph $G_{n, 2 p-p^{2}}$ which determines the connected component of vertex $\langle x\rangle$. Let the "current graph" be that subgraph of $D_{F}$ which consists of the vertices and edges that have been examined by the local search. The "frontier" consists of those vertices of the current graph from which further searching may be done. Initially the current graph consists of just the literal $x$, and $x$ is in the frontier. Eventually the frontier will be empty, terminating the local search, at which point $\widetilde{D}_{F}^{+}(x)$ will be defined to be the current graph. During the search, certain edges $v \rightarrow w$ will be tested to see if they are in $D_{F}$, and the search records whether the results are "yes" or "no" on the corresponding unoriented edge $\langle v\rangle \sim\langle w\rangle$. These test results will later be used to construct the random graph $G_{n, 2 p-p^{2}}$. Each step in the local search consists of the substeps listed below.

1. An arbitrary literal $v$ in the frontier is selected (one choice is the lexicographically smallest).

2. For each literal $w$ such that neither $w$ nor $\bar{w}$ is in the current graph, check if $v \rightarrow w$ is in $D_{F}$, and record either "yes" or "no" on the edge $\langle v\rangle \sim\langle w\rangle$ accordingly.

3. For each literal $w$ for which "yes" was recorded, declare $w$ to be a "new literal" unless "yes" is recorded for both $w$ and $\bar{w}$, in which case we declare only one (say the unnegated one) of them to be a "new literal."

4. Adjoin each new literal $w$ and the edge $v \rightarrow w$ to the current graph.

5. Adjoin each new literal $w$ to the frontier. Remove $v$ from the frontier.

6. Consider each ordered pair of vertices $(w, f)$ such that either $(1) w$ is new and $f$ is in the frontier but not new, or (2) $w$ and $f$ are both new, and $w$ is lexicographically smaller. Test if $w \rightarrow f$ or $f \rightarrow w$ in $D_{F}$, and record either "yes" or "no" on the edge $\langle w\rangle \sim\langle f\rangle$ accordingly. If there is one "yes," adjoin the corresponding edge to the current graph, if there are two "yes"'s, adjoin only one of the edges, say the edge $w \rightarrow f$.

Lemma 2.6. The trimmed out-graph $\widetilde{D}_{F}^{+}(x)$ defined above has the following properties.

i) $\widetilde{D}_{F}^{+}(x)$ is a subgraph of $D_{F}^{+}(x)$.

ii) $\widetilde{L}_{F}^{+}(x)$ is strictly distinct.

iii) $\widetilde{L}_{F}^{+}(x)=L_{F}^{+}(x)$ if and only if $L_{F}^{+}(x)$ is strictly distinct.

iv) For $F=F_{n, p}$, the unoriented projection of the digraph $\widetilde{D}_{F}^{+}(x)$ has the same distribution as $C_{n, 2 p-p^{2}}(x)$. In particular, $\left|\widetilde{L}_{F_{n, p}}^{+}(x)\right|$ and $\left|C_{n, 2 p-p^{2}}(x)\right|$ are equidistributed.

Proof. By construction, properties (i) and (ii) are obvious. Property (iii) is not much more difficult. There are certain possible edges leading out of the vertex set $\widetilde{L}_{F}^{+}(x)$ that were never tested, or that were tested and present, but then excluded from the trimmed out-graph $\widetilde{D}_{F}^{+}(x)$ anyway. But each such edge either led to a literal already in $\widetilde{L}_{F}^{+}(x)$, or else led to a literal whose complement was in $\widetilde{L}_{F}^{+}(x)$. Thus if the literal set $L_{F}^{+}(x)$ were to contain more literals than $\widetilde{L}_{F}^{+}(x)$, then $L_{F}^{+}(x)$ would not be strictly distinct. On the 
other hand, if $L_{F}^{+}(x)$ and $\widetilde{L}_{F}^{+}(x)$ are identical, then $L_{F}^{+}(x)$ is trivially strictly distinct by property (ii).

Property (iv) is similarly easy. First, for each literal $u \in[n]$, we define $[u]=\{u, \bar{u}\}$. By induction we shall prove that, at the beginning and end of each step of the search, the following properties hold:

1. For every pair of literals $u$ and $v$ of the current graph, precisely two edges between $[u]$ and $[v]$ have been tested.

2. For every literal $v$ in the current graph but not in the frontier, and every literal $w$ such that neither $w$ nor $\bar{w}$ is in the current graph, precisely two edges between $[v]$ and $[w]$ have been tested, both results being "no."

3. For every literal $v$ in the frontier, and any literal $w$ such that neither $w$ nor $\bar{w}$ is in the current graph, none of the edges between $[v]$ and $[w]$ have been tested.

4. If none of $u, \bar{u}, v, \bar{v}$ are in the current graph, then no edges between $[u]$ and $[v]$ have been tested.

5. For any pair of strictly distinct literals $u, v \in[n]$, either none or precisely one of the four edges between $[u]$ and $[v]$ appears in the current graph. The latter happens if and only if some test between $[u]$ and $[v]$ was positive.

Indeed, assume that (1) - (5) hold at the beginning of a step. To prove that (1) holds at the end of the step, we first note that no edge between $[u]$ and $[v]$ was tested in the current step if neither $u$ nor $v$ is new. If $v$ is old and $u$ is new, then either $v$ was the selected vertex in the frontier, in which case the edges $v \rightarrow u$ and $v \rightarrow \bar{u}$ have been tested in the current step, or $v$ was not in the frontier, in which case precisely two edges between $[u]$ and $[v]$ were tested in a previous step (with answer "no") by the inductive assumption (2). If both $v$ and $u$ are new, then no edge between $[u]$ and $[v]$ was tested in a previous step by the inductive assumption (3), and precisely two edges (the edges $u \rightarrow v$ and $v \rightarrow u$ ) between $[u]$ and $[v]$ are tested in the current step.

To prove (2), we note that if $v$ is in the current graph but not in the frontier, it was in the frontier in some previous step, and got removed from the frontier after all edges from $v$ to vertices $u$, with neither $u$ nor $\bar{u}$ in the current graph at the time, were tested. This includes in particular the vertex $w$ in question, and since we assume that neither $w$ nor $\bar{w}$ is in the current graph, it follows that both tests must have given the result "no" at the time. After that step, $v$ is not in the frontier, so no edge containing $v$ or $\bar{v}$ is ever tested again, implying statement (2).

Statement (3) follows from the observation that an edge between a vertex $v$ in the current graph and a vertex $w$ such that neither $w$ nor $\bar{w}$ is in the current graph is only tested if $v$ is the selected vertex in the current step, in which case it is not in the frontier after this step anymore.

Statement (4) is obvious, since an edge $f \rightarrow w$ is only tested if either $f$ is in the frontier (and hence in the current graph before the current step), or both $f$ and $w$ are new vertices, which means they are in the current graph after steps (1) - (6). 
To prove (5), we consider three cases. In the first case, none of the vertices $u, \bar{u}, v$ and $\bar{v}$ is in the current graph, in which case no edge between $[u]$ and $[v]$ appears in the current graph by the inductive assumption (4). The second case is the one in which exactly one of the four vertices $u, \bar{u}, v$ and $\bar{v}$ is in the current graph. Without loss of generality, let us assume that this is the vertex $v$. Then none of the edges between $[u]$ and $[v]$ appears in the current graph by (2) and (3). The third case is that precisely two of the four vertices $u, \bar{u}, v$ and $\bar{v}$ are in the current graph, say $u$ and $v$. Then precisely two of the four edges between $[u]$ and $[v]$ have been tested by the inductive assumption (1). Since the above search procedure always tests two of the four edges between $[u]$ and $[v]$ at a given time, and adds one (but not both) of them precisely when at least one of them tests positive, we get (4).

We now use the properties (1) - (5) above to prove statement (iv) of the lemma. If we pick the unordered pairs of numbers between 1 and $n$ in some arbitrary order, each time randomly saying "present" (with probability $2 p-p^{2}$ ) or "absent" (with probability $\left.(1-p)^{2}\right)$, then even if the order in which we pick the pairs depends on the previous random choices of present/absent, the result will be the random graph $G_{n, 2 p-p^{2}}$. This is in effect what the trimmed local search does, except that it stops when the connected component containing $\langle x\rangle$ has been determined. Thus unoriented projection of $\widetilde{D}_{F}^{+}(x)$ is just the connected component containing $\langle x\rangle$ in $G_{n, 2 p-p^{2}}$.

\section{Strategy of the Proof}

In this section, we shall first state our principal estimates and results for the distribution $F_{n, p}$ (to be proved in later sections), and then give the heuristics for these results.

3.1. Main Results For the Distribution $F_{n, p}$. As explained in the last section, the spine of a formula $F$ consists of all literals $x$ for which $x \rightsquigarrow \bar{x}$ (see Lemma 2.3), which in turn is just the set of all literals $x$ such that $L_{F}^{+}(x)$ is strictly distinct (see Lemma 2.4). If $F$ is distributed according to the model $F_{n, p}$, the expectation and variance of the size of $S\left(F_{n, p}\right)$ are therefore given by the equations

$$
E\left(\left|S\left(F_{n, p}\right)\right|\right)=\sum_{x \in[n]} \mathbb{P}\left(x \underset{F_{n, p}}{\rightsquigarrow} \bar{x}\right)
$$

and

$$
E\left(\left|S\left(F_{n, p}\right)\right|^{2}\right)-E\left(\left|S\left(F_{n, p}\right)\right|\right)^{2}=\sum_{x, y \in[n]} \mathbb{P}\left(x \underset{F_{n, p}}{x} \bar{x} \text { and } y \underset{F_{n, p}}{\rightsquigarrow} \bar{y}\right)-\mathbb{P}\left(x \underset{F_{n, p}}{x} \bar{x}\right) \mathbb{P}\left(y \underset{F_{n, p}}{\rightsquigarrow} \bar{y}\right),
$$

where $x \underset{F_{n, p}}{\rightsquigarrow} \bar{x}$ a shorthand for $x \underset{D_{F_{n, p}}}{\rightsquigarrow} \bar{x}$.

The following two theorems allow us to prove suitable bounds on the expected size and variance of the spine of a random 2-SAT formula, and are at the heart of our proofs. Before we can proceed, we unfortunately need a short interlude on Landau symbols: 
In this paper, we shall use Landau's notation $f=O(g)$ and $f=o(g)$. As usual, $f=O(g)$ stands for a bound $|f| \leq c|g|$, where $c$ is a universal constant, unless otherwise specified. If we have a bound of the form $|f| \leq h(g)|g|$, where $h(g)$ is a function which is bounded above, though not necessarily uniformly, for finite $g$, and which is uniformly bounded above as $g$ goes to zero, we shall use the notation $f=\mathbb{O}_{0}(g)$. In this notation, $e^{x^{2}}-1$ is $\mathbb{O}_{0}\left(x^{2}\right)$, but it is not $O\left(x^{2}\right)$.

Our use of the symbol $o(g)$ is slightly stronger than usual. Typically, $f=o(g)$ means that $f / g$ goes to zero as the independent variables in question tend to their limiting values, but usually $f=o(g)$ does not require that $f / g$ is bounded in the whole domain of the independent variables. We require both uniform boundedness and that $f / g$ tends to zero. Since it may be ambiguous which independent variables tend to $\infty$ or 0 in an expression of the form $f=o(g)$, we frequently specify the variables in question. Thus $f=o_{\lambda, \varepsilon}(g)$ means that $f / g \rightarrow 0$ as $\lambda \rightarrow \infty$ and $\varepsilon \rightarrow 0$. For example, in this notation, the $o(1)$ terms in Theorem 1.4 would be written as $o_{\varepsilon, \lambda_{n}}(1)$.

Finally, as mentioned earlier, $f=\Theta(g)$ means that there exist positive, finite constants $c_{1}$ and $c_{2}$ such that $c_{1} \leq f / g \leq c_{2}$. Unless noted otherwise, these constants are universal.

Theorem 3.1. There are constants $\lambda_{0}$ and $\varepsilon_{0}, 0<\lambda_{0}<\infty$ and $0<\varepsilon_{0}<1$, such that the following statements hold for

$$
p=\frac{1}{2 n}(1+\varepsilon)=\frac{1}{2 n}\left(1+\lambda_{n} n^{-1 / 3}\right)
$$

and $\lambda_{0} \leq\left|\lambda_{n}\right| \leq \varepsilon_{0} n^{1 / 3}$.

i) If $\varepsilon<0$, then

$$
\mathbb{P}\left(x \underset{F_{n, p}}{\rightsquigarrow} \bar{x}\right)=\frac{n^{-1 / 3}}{4 \lambda_{n}^{2}}\left(1+o_{\varepsilon, \lambda_{n}}(1)\right) .
$$

ii) If $\varepsilon>0$, then

$$
\begin{aligned}
\mathbb{P}\left(x \underset{F_{n, p}}{x} \bar{x}\right) & =\vartheta(\varepsilon)\left(1+o_{\lambda_{n}}(1)\right), \\
& =2 \lambda_{n} n^{-1 / 3}\left(1+o_{\lambda_{n}}(1)+O(\varepsilon)\right)
\end{aligned}
$$

where $\vartheta(\varepsilon)$ is defined in (1.8).

Theorem 3.2. Let $p, \varepsilon$ and $\lambda_{n}$ be as in Theorem 3.1. Then the following statements hold for all strictly distinct literals $x$ and $y$.

i) If $\varepsilon<0$, then

$$
\mathbb{P}\left(x \underset{F_{n, p}}{\rightsquigarrow} \bar{x}\right) \mathbb{P}\left(y \underset{F_{n, p}}{\rightsquigarrow} \bar{y}\right) \leq \mathbb{P}\left(x \underset{F_{n, p}}{\rightsquigarrow} \bar{x} \text { and } y \underset{F_{n, p}}{\rightsquigarrow} \bar{y}\right)=O\left(\frac{n^{-2 / 3}}{\lambda_{n}^{4}}\right) .
$$

ii) If $\varepsilon>0$, then

$$
0 \leq \mathbb{P}\left(x \underset{F_{n, p}}{\rightsquigarrow} \bar{x} \text { and } y \underset{F_{n, p}}{\rightsquigarrow} \bar{y}\right)-\mathbb{P}\left(x \underset{F_{n, p}}{\rightsquigarrow} \bar{x}\right) \mathbb{P}\left(y \underset{F_{n, p}}{\rightsquigarrow} \bar{y}\right)=O\left(\frac{n^{-2 / 3}}{\lambda_{n}}\right) .
$$


Remark 3.3. By monotonicity, the bound (3.6) can be extended to all $\lambda_{n} \in\left[-n^{1 / 3},-\lambda_{0}\right]$. Indeed, using that the events $x \rightsquigarrow \bar{x}$ and $y \rightsquigarrow \bar{y}$ are monotone events, we have that

$$
\mathbb{P}\left(x \underset{F_{n, p}}{\rightsquigarrow} \bar{x} \text { and } y \underset{F_{n, p}}{\rightsquigarrow} \bar{y}\right) \leq \mathbb{P}\left(x \underset{F_{n, p_{0}}}{\rightsquigarrow} \bar{x} \text { and } y \underset{F_{n, p_{0}}}{\rightsquigarrow} \bar{y}\right)
$$

provided $p \leq p_{0}$. Setting $p_{0}=\left(1-\varepsilon_{0}\right) / 2 n$, using equation (3.6) to bound the right hand side by $O\left(n^{-2 / 3} /\left(n^{1 / 3}\right)^{4}\right)$, and observing that $n^{-2 / 3} /\left(n^{1 / 3}\right)^{4}=O\left(n^{-2 / 3} / \lambda_{n}^{4}\right)$ provided $\lambda_{n} \in\left[-n^{1 / 3},-\varepsilon_{0} n^{1 / 3}\right]$, we obtain that

$$
\mathbb{P}\left(x \underset{F_{n, p}}{\rightsquigarrow} \bar{x} \text { and } y \underset{F_{n, p}}{\rightsquigarrow} \bar{y}\right)=O\left(\frac{n^{-2 / 3}}{\lambda_{n}^{4}}\right) \text { for all } \lambda_{n} \in\left[-n^{1 / 3},-\lambda_{0}\right] .
$$

Given the above two theorems, we shall prove the following analogue of Theorem 1.1 for the ensemble $F_{n, p}$.

Theorem 3.4. There are constants $\lambda_{0}$ and $\varepsilon_{0}, 0<\lambda_{0}<\infty$ and $0<\varepsilon_{0}<1$, such that the following statements hold for $p=\frac{1}{2 n}\left(1+\lambda_{n} n^{-1 / 3}\right)$ and $\lambda_{0} \leq\left|\lambda_{n}\right| \leq \varepsilon_{0} n^{1 / 3}$.

i) If $\lambda_{n}<0$, then

$$
\mathbb{P}\left(F_{n, p} \text { is } \mathrm{SAT}\right)=\exp \left(-\Theta\left(\left|\lambda_{n}\right|^{-3}\right)\right) .
$$

ii) If $\lambda_{n}>0$, then

$$
\mathbb{P}\left(F_{n, p} \text { is } \mathrm{SAT}\right)=\exp \left(-\Theta\left(\lambda_{n}^{3}\right)\right) .
$$

For fixed $\lambda_{n}$, both (3.4) and (3.5) are of the form $\mathbb{P}(x \rightsquigarrow \bar{x})=\Theta\left(n^{-1 / 3}\right)$. Together with equation (3.1), Theorem 3.1 therefore implies that the expected size of the spine scales like $n^{2 / 3}$, provided $\lambda_{n}$ stays bounded as $n \rightarrow \infty$. The heuristics for this scaling with $n$ will be given in the next subsection, and the actual proof of the scaling will be given in Sections 5 7. Theorem 3.2 allows us to control the deviations of the random variable $\left|S\left(F_{n, p}\right)\right|$ from its expectation; its proof will be given in Section 8 . Together, these two theorems allow us to prove Theorem 3.4, which is just the analogue of Theorem 1.1 in the model $F_{n, p}$. In the final subsection, we shall describe the strategy of this proof. While the actual proof is easier in the model $F_{n, p}$, the heuristic argument is easier in the model $F_{n, m}$. Our goal in the last subsection is therefore to describe how the scaling $n^{2 / 3}$ for the size of the spine in the model $F_{n, m}$ leads to bounds of the form (3.9) and (3.10). The actual proof of Theorem 3.4 is given in Section 4 .

3.2. Heuristics for the Scaling of the Spine. The proof of Theorem 3.1 (and hence also the proof of the expected size of the spine, Theorem 1.4) uses the digraph representation of the last section. Indeed, by Lemmas 2.3, 2.4, 2.6 (iii) and 2.6 (iv), and the fact the probability of the event $x \rightsquigarrow \bar{x}$ does not depend on the choice of the literal 
$x \in[n]$, we have

$$
\begin{aligned}
E\left(\left|S\left(F_{n, p}\right)\right|\right) & =2 n \mathbb{P}(x \rightsquigarrow \bar{x}) \\
& =2 n \mathbb{P}\left(L_{F_{n, p}}^{+}(x) \neq \widetilde{L}_{F_{n, p}}^{+}(x)\right) \\
& =2 n \sum_{k=1}^{n}\left[\mathbb{P}\left(\left|\widetilde{L}_{F_{n, p}}^{+}(x)\right|=k\right)-\mathbb{P}\left(\left|L_{F_{n, p}}^{+}(x)\right|=k, L_{F_{n, p}}^{+}(x) \text { is s.d }\right)\right] \\
& =2 n \sum_{k=1}^{n}\left[\mathbb{P}\left(\left|C_{n, 2 p-p^{2}}(x)\right|=k\right)-\mathbb{P}\left(\left|L_{F_{n, p}}^{+}(x)\right|=k, L_{F_{n, p}}^{+}(x) \text { is s.d }\right)\right] .
\end{aligned}
$$

It turns out that for $2 p-p^{2}$ near to the random graph threshold $1 / n$, and $k \leq \Theta\left(n^{2 / 3}\right)$, the size of the largest component in the random graph, the probability that $L_{F_{n, p}}^{+}(x)$ is strictly distinct and has size $k$ is well approximated by $\mathbb{P}\left(\left|C_{n, 2 p-p^{2}}(x)\right|=k\right)$, so that the summand in equation (3.11) is approximately zero. On the other hand, for $2 p-p^{2}$ near $1 / n$ and $k \geq \Theta\left(n^{2 / 3}\right)$, only the sum over $\mathbb{P}\left(\left|C_{n, 2 p-p^{2}}(x)\right|=k\right)$ contributes to (3.11). Thus we can approximate

$$
E\left(\left|S\left(F_{n, p}\right)\right|\right) \approx 2 n \mathbb{P}\left(\left|C_{n, 2 p-p^{2}}(x)\right| \geq n^{2 / 3}\right) .
$$

As the reader might imagine, the above arguments require a good deal of justification; see Section 507 for precise bounds. But for $2 p-p^{2}$ near $1 / n$, the probability that $\left|C_{n, 2 p-p^{2}}(x)\right| \geq n^{2 / 3}$ scales like the probability that $x$ lies in the largest component in the random graph, which in turn scales like $n^{-1 / 3}$ (see e.g. [Bol85]). This implies that the expected size of the spine $S\left(F_{n, p}\right)$ scales like $n^{2 / 3}$ provided $p$ is of the form $p=\frac{1}{2 n}\left(1 \pm \Theta\left(n^{-1 / 3}\right)\right)$. Observing that the models $F_{n, p}$ and $F_{n, m}$ are equivalent as long as $m$ is near its expected value $\left(\begin{array}{l}n \\ 2\end{array}\right) p$ (see Appendix A), we obtain the scaling of Theorem 1.4.

3.3. Heuristics for the Scaling of the Window. As explained earlier, our goal is to describe how the behavior $E\left(\left|S\left(F_{n, m}\right)\right|\right)=\Theta\left(n^{2 / 3}\right)$ leads to the bounds (3.9) and (3.10). To this end, consider a process which builds random formulas as follows: Given a 2-SAT formula $F_{m}$, let $F_{m+1}=F_{m} \wedge C$, where $C=x \vee y$ is chosen uniformly at random from the set of all 2-clauses over $\left\{x_{1}, \ldots, x_{n}\right\}$ that have not yet been used in $F_{m}$. Obviously, the distribution of $F_{m}$ is the same as that of $F_{n, m}$. Furthermore, $F_{m+1}$ is satisfiable if and only if $F_{m}$ is satisfiable and either $x$ or $y$ does not lie in the spine of $F_{m}$. Conditioned on the events that $F_{m}$ is SAT and that $S\left(F_{m}\right)$ has size $s$, the probability that $F_{m+1}$ is SAT is therefore equal to

$$
\begin{aligned}
\mathbb{P}\left(\operatorname{SAT}\left(F_{m+1}\right) \mid \operatorname{SAT}\left(F_{m}\right) \text { and }\left|S\left(F_{m}\right)\right|=s\right) & =1-\left(\begin{array}{l}
s \\
2
\end{array}\right)\left(4\left(\begin{array}{l}
n \\
2
\end{array}\right)-m\right)^{-1} \\
& =1-\frac{s(s-1)}{4 n(n-1)-2 m} .
\end{aligned}
$$


By the analogue of Theorem 3.1 for the model $F_{n, m}$ and the monotonicity of the expected size of the spine, we have that near the transition point, $E(|S(F)|)=\Theta\left(n^{2 / 3}\right)$. Neglecting the difference between statements in expectation and statements in probability, equation (3.13) therefore implies that the probability that $F_{m+1}$ is UNSAT, conditioned on $F_{m}$ being satisfiable, is $\Theta\left(n^{4 / 3} / n^{2}\right)=\Theta\left(n^{-2 / 3}\right)$. After $\Theta\left(n^{2 / 3}\right)$ steps, a satisfiable formula therefore becomes UNSAT, giving the a finite-size scaling window of width $\Theta\left(n^{2 / 3}\right)$ in $m$, and hence of width $\Theta\left(n^{-1 / 3}\right)$ in $m / n$. This is the result in Corollary 1.2 .

In order to explain heuristically the error bounds $\exp \left(-\Theta\left(\left|\lambda_{n}\right|^{-3}\right)\right)$ and $\exp \left(-\Theta\left(\lambda_{n}^{3}\right)\right)$ in Theorem 1.1, we proceed as follows. As a consequence of (3.13), we have that

$$
\mathbb{P}\left(\operatorname{SAT}\left(F_{m+1}\right) \mid \operatorname{SAT}\left(F_{m}\right)\right)=1-\frac{E\left(\left(\left|S\left(F_{m}\right)\right|^{2}-\left|S\left(F_{m}\right)\right|\right) \mid \operatorname{SAT}\left(F_{m}\right)\right)}{4 n(n-1)-2 m},
$$

and hence

$$
\mathbb{P}\left(\operatorname{SAT}\left(F_{m}\right)\right)=\prod_{k=0}^{m-1}\left(1-\frac{E\left(\left(\left|S\left(F_{k}\right)\right|^{2}-\left|S\left(F_{k}\right)\right|\right) \mid \operatorname{SAT}\left(F_{k}\right)\right)}{4 n(n-1)-2 k}\right) .
$$

Neglecting the difference between conditional and unconditional expectations, and approximating $\left|S\left(F_{k}\right)\right|^{2}-\left|S\left(F_{k}\right)\right|$ by $\left|S\left(F_{k}\right)\right|^{2}$, we get

$$
\begin{aligned}
\mathbb{P}\left(\operatorname{SAT}\left(F_{m}\right)\right) & \approx \prod_{k=0}^{m-1}\left(1-\frac{1}{4 n^{2}-2 k} E\left(\left|S\left(F_{k}\right)\right|^{2}\right)\right) \\
& \approx \exp \left(-\sum_{k=0}^{m-1} \frac{1}{4 n^{2}} E\left(\left|S\left(F_{k}\right)\right|^{2}\right)\right) .
\end{aligned}
$$

For $\lambda_{n}<0$ and $k=\left(1+\lambda n^{-1 / 3}\right) n, \lambda \in\left(-n^{1 / 3}, \lambda_{n}\right)$, we then approximate $E\left(\left|S\left(F_{k}\right)\right|^{2}\right)$ by $E\left(\left|S\left(F_{n, p}\right)\right|^{2}\right), p=\left(1+\lambda n^{-1 / 3}\right) / 2 n$. Using Theorems 3.1 and 3.2 to estimate this probability, we have

$$
\begin{aligned}
\mathbb{P}\left(\operatorname{SAT}\left(F_{m}\right)\right) & \approx \exp \left(-\frac{1}{4 n^{2}} \int_{k=0}^{m} d k E\left(\left|S\left(F_{k}\right)\right|\right)^{2}\right) \\
& \approx \exp \left(-\frac{1}{4 n^{2}} \int_{-n^{1 / 3}}^{\lambda_{n}} n^{2 / 3} d \lambda \Theta\left(n^{2 / 3} / \lambda^{2}\right)^{2}\right) \\
& =\exp \left(\Theta\left(\left|\lambda_{n}\right|^{-3}\right)\right),
\end{aligned}
$$

giving the bound (1.4) below the threshold. In a similar way, we can integrate the bound (3.5) to obtain a heuristic derivation of (1.4) above the threshold.

The actual proof of Theorem 1.1 in the form (3.9)-(3.10) will be given in the next section, and relies heavily on Theorems 3.1 and 3.2, which in turn are proven in Sections 58. In addition, we shall need two more technical lemmas, to be proven in Section 9. In Appendix A, we discuss the relation between the models $F_{n, m}$ and $F_{n, p}$. 


\section{Probability of Satisfiability}

In this section we prove Theorem 3.4, which together with Appendix A establishes Theorem 1.1. The lower bounds depend on the second moment estimates of Theorem 3.2, which is proved in Section 8. The upper bounds depends on a theorem showing that with high probability there are many structures, to be called hourglasses, that are "seeds" for the growth of the spine. The hourglass theorem is proven in Section 9, after we develop suitable machinery in the intervening sections.

To derive the bounds, we shall find it convenient to view the random formula $F_{n, p}$ as a process, and to couple the processes for all possible values of $n$ and $p$. To do this, for each unordered pair of natural numbers we pick four random variables uniformly distributed on the interval from 0 to 1 , so that the set of all these random variables indexed by 4 copies of $\left(\begin{array}{c}\mathbb{N} \\ 2\end{array}\right)$ (sometimes denoted $\mathbb{N}^{(2)}$ ) is a set of independent random variables. As usual $\left(\begin{array}{c}X \\ 2\end{array}\right)$ denotes the set of unordered pairs of elements of the set $X$. A pair of natural numbers specify two different variables of the formula, and the four random variables associated with the pair correspond to the four different clauses that can be made using these variables, so that each possible clause $C$ has its own independent random variable $U_{C}$ distributed uniformly in $[0,1]$. A clause $C$ appears in the formula on $n$ variables at probability $p$ precisely when the indices of its two variables are not larger than $n$, and $U_{C}<p$. We shall call $U_{C}$ the birthday of the clause $C$. The process $\widetilde{F}$ is the collection of all these random variables, and it defines a family of formulas by

$$
F_{n, p}=\bigwedge_{C: n(C) \leq n, U_{C}<p} C,
$$

where $n(C)$ denotes the maximum index of the two variables in clause $C$. It is easy to see that for each value of $n$ and $p$, the distribution of the resulting formula $F_{n, p}$ is exactly the distribution introduced before, which justifies our using the same notation as before. By construction, satisfiability of $F_{n, p}$ is monotone decreasing in $n$ and $p$.

LOWER BOUNDS. To derive the lower bounds of Theorem 1.1, it is sufficient to consider the coupling above for a fixed value of $n$, so we suppress the variable $n$. Given $\widetilde{F}$ (i.e., $F_{p}$ for each $p$ ), we define the reduced formula process $\widetilde{\Phi}=\left(\Phi_{p}\right)_{p \in[0,1]}$ as follows: $\Phi_{0}$ has no clauses. Start with $p=0$, and increase it until $p=1$. Clauses are added to $F_{p}$ one at a time; each time we add a clause to $F_{p}$, we also add that clause to $\Phi_{p}$ provided that doing so does not make $\Phi_{p}$ unsatisfiable.

Given $\widetilde{\Phi}$ we can define a new formula process $\widetilde{H}$ as follows: For each clause $C$ appearing in $\widetilde{\Phi}$, set its birthday in $\widetilde{H}$ to coincide with its birthday in $\widetilde{\Phi}$. For each clause $C$ not appearing in $\widetilde{\Phi}_{1}$, there is some smallest value $p_{\text {min }}$ of $p$ for which $\Phi_{p} \wedge C$ is not satisfiable. Pick the birthday of $C$ in $H_{p}$ uniformly at random in the interval $\left[p_{\min }, 1\right)$. Since the $\widetilde{H}$ process is drawn uniformly at random from the set of $\widetilde{F}$ processes with reduced formula process $\widetilde{\Phi}$, the $\widetilde{H}$ and $\widetilde{F}$ processes are identically distributed. Also note that the first time that $H_{p}$ differs from $\Phi_{p}$ is also the first time that $H_{p}$ becomes unsatisfiable. 
Let $U(F)$ denote the number of different clauses $C$ such that $F \wedge C$ is unsatisfiable. If $F$ is itself satisfiable, then

$$
U(F)=\left(\begin{array}{c}
|S(F)| \\
2
\end{array}\right)
$$

where as usual $S(F)$ denotes the spine of $F$.

Conditional on the reduced formula process being $\widetilde{\Phi}$ and $H_{p}$ being satisfiable (i.e. that $\left.H_{p}=\Phi_{p}\right)$, the probability that $H_{p+\delta}$ is satisfiable is

$$
\left(1-\frac{\delta}{1-p}\right)^{U\left(\Phi_{p}\right)}=\exp \left[-\frac{U\left(\Phi_{p}\right)}{1-p} \delta+O\left(\delta^{2}\right)\right]
$$

provided $\delta$ is small enough. Multiplying these probabilities for $p=0, \delta, 2 \delta, \ldots$, and passing to the limit $\delta \rightarrow 0$, we find that conditional upon $\widetilde{\Phi}$, the probability that $H_{p}$ is satisfiable is given by

$$
\mathbb{P}\left[\operatorname{SAT}\left(H_{p}\right) \mid \widetilde{\Phi}\right]=\exp \left[-\int_{0}^{p} \frac{U\left(\Phi_{s}\right)}{1-s} d s\right]
$$

We have

$$
\begin{aligned}
\mathbb{P}\left[\operatorname{SAT}\left(F_{p}\right)\right] & =\mathbb{P}\left[\operatorname{SAT}\left(H_{p}\right)\right] \\
& =E_{\widetilde{\Phi}}\left[\mathbb{P}\left[\operatorname{SAT}\left(H_{p}\right) \mid \widetilde{\Phi}\right]\right] \\
& =E_{\widetilde{\Phi}}\left[\exp \left[-\int_{0}^{p} \frac{U\left(\Phi_{s}\right)}{1-s} d s\right]\right] \\
& \geq \exp E_{\widetilde{\Phi}}\left[-\int_{0}^{p} \frac{U\left(\Phi_{s}\right)}{1-s} d s\right] \\
& =\exp \left[-\int_{0}^{p} \frac{E_{\widetilde{\Phi}}\left[U\left(\Phi_{s}\right)\right]}{1-s} d s\right] \\
& =\exp \left[-\int_{0}^{p} \frac{4\left(\begin{array}{l}
n \\
2
\end{array}\right) \mathbb{P}\left[x \rightsquigarrow \bar{x} \text { and } y \rightsquigarrow \bar{y} \text { in } \Phi_{s}\right]}{1-s} d s\right] \\
& \geq \exp \left[-4\left(\begin{array}{l}
n \\
2
\end{array}\right) \int_{0}^{p} \frac{\mathbb{P}\left[x \rightsquigarrow \bar{x} \text { and } y \rightsquigarrow \bar{y} \text { in } F_{s}\right]}{1-s} d s\right],
\end{aligned}
$$

where $x$ and $y$ are fixed strictly distinct literals.

Next we proceed to estimate the integral. Since we are principally interested in the case $p=O(1 / n)$, let us assume $p=o_{n}(1)$ so that the effect of the denominator of the integrand is negligible. Recalling that $p$ and $\lambda_{n}$ are related by (3.3), and setting

$$
s=s(t)=\frac{1+t n^{-1 / 3}}{2 n}
$$


we get

$$
\mathbb{P}\left[\operatorname{SAT}\left(F_{p}\right)\right] \geq \exp \left[-\left(1+o_{n}(1)\right) n^{2 / 3} \int_{-n^{1 / 3}}^{\lambda_{n}} \mathbb{P}\left[x \rightsquigarrow \bar{x} \text { and } y \rightsquigarrow \bar{y} \text { in } F_{s(t)}\right] d t\right] .
$$

Remark 4.1. It is not hard to derive the analogue of (4.2) in the model $F_{n, m}$. Indeed, starting from (3.15), rewriting

$$
E\left(\left|S\left(F_{k}\right)\right|^{2}-\left|S\left(F_{k}\right)\right| \mid \operatorname{SAT}\left(F_{k}\right)\right)=4\left(\begin{array}{l}
n \\
2
\end{array}\right) \mathbb{P}\left(x \rightsquigarrow \bar{x} \text { and } y \rightsquigarrow \bar{y} \text { in } F_{k} \mid \operatorname{SAT}\left(F_{k}\right)\right),
$$

where $x$ and $y$ are strictly distinct, and observing that by the FKG inequality,

$$
\mathbb{P}\left(x \rightsquigarrow \bar{x} \text { and } y \rightsquigarrow \bar{y} \text { in } F_{k} \mid \operatorname{SAT}\left(F_{k}\right)\right) \leq \mathbb{P}\left(x \rightsquigarrow \bar{x} \text { and } y \rightsquigarrow \bar{y} \text { in } F_{k}\right),
$$

one gets

$$
\mathbb{P}\left(\operatorname{SAT}\left(F_{n, m}\right)\right) \geq \prod_{k=0}^{m-1}\left[1-\left(1+O\left(m / n^{2}\right)\right) \mathbb{P}\left(x \rightsquigarrow \bar{x} \text { and } y \rightsquigarrow \bar{y} \text { in } F_{n, k}\right)\right] .
$$

Proof of the lower bound of Theorem 3.4 in the subcritical regime. For $t \in\left[-n^{1 / 3},-\lambda_{0}\right]$, the probability in the integrand in equation (4.2) is $O\left(n^{-2 / 3} / t^{4}\right)$ by Theorem 3.2 and Remark 3.3. Integrating, we find that

$$
\mathbb{P}\left[\operatorname{SAT}\left(F_{p}\right)\right] \geq \exp \left[\Theta\left(\frac{1}{\lambda_{n}{ }^{3}}\right)\right]=1-\Theta\left(\frac{1}{\left|\lambda_{n}\right|^{3}}\right)
$$

provided $\lambda_{n} \in\left[-n^{-1 / 3},-\lambda_{0}\right]$.

Proof of the lower bound of Theorem 3.4 in the supercritical regime. By Theorems 3.1 and 3.2 , the probability in the integrand in equation (4.2) is $4 n^{-2 / 3} t^{2}\left(1+O(\varepsilon)+o_{\lambda_{n}}(1)\right)$, provided $t \in\left[\lambda_{0}, \varepsilon_{0} n^{1 / 3}\right]$. In the middle region $t \in\left[-\lambda_{0}, \lambda_{0}\right]$ we upper bound the probability in the integrand by $\Theta\left(n^{-2 / 3}\right)$, and we bound it in the left region $t \in\left[-n^{1 / 3},-\lambda_{0}\right]$ by $O\left(n^{-2 / 3} / t^{4}\right)$ as above. Integrating, we find that

$$
\mathbb{P}\left[\operatorname{SAT}\left(F_{p}\right)\right] \geq \exp \left[-\frac{4+o_{\varepsilon, \lambda_{n}}(1)}{3} \lambda_{n}^{3}\right] .
$$

provided $\lambda_{n} \in\left[\lambda_{0}, \varepsilon_{0} n^{1 / 3}\right]$.

UPPER BOUNDS. To bound from above the probability of satisfiability, we use Theorem 4.3 below, which states that with high probability there are certain types of structures contained within the directed graph associated with a formula.

Definition 4.2. An hourglass is a triple $\left(v, I_{v}, O_{v}\right)$ where $v$ is a literal, and $I_{v}$ and $O_{v}$ are two disjoint sets of literals not containing $v$, such that for each $x \in I_{v}$, there is a path $x \rightsquigarrow v$ in $I_{v} \cup\{v\}$, and for each $y \in O_{v}$, there is a path $v \rightsquigarrow y$ in $O_{v} \cup\{v\}$. Furthermore, we require that $\{v\} \cup I_{v} \cup O_{v}$ is strictly distinct. We call $v$ the central literal, $I_{v}$ the in-portion, and $O_{v}$ the out-portion of the hourglass. 
Theorem 4.3. There are constants $\lambda_{0}$ and $\varepsilon_{0}, 0<\lambda_{0}<\infty$ and $0<\varepsilon_{0}<1$, such that for $p=\frac{1}{2 n}\left(1+\lambda_{n} n^{-1 / 3}\right)$ and $\lambda_{0} \leq\left|\lambda_{n}\right| \leq \varepsilon_{0} n^{1 / 3}$, the following statements hold with probability at least $1-\exp \left(-\Theta\left(\left|\lambda_{n}\right|^{3}\right)\right.$.

i) If $\lambda_{n}<0$, then there are at least $\Theta\left(\left|\lambda_{n}\right|^{3}\right)$ disjoint, mutually strictly distinct hourglasses with in-portion and out-portion each of size at least $n^{2 / 3} / \lambda_{n}^{2}$.

ii) If $\lambda_{n}>0$, then there is at least one hourglass with in-portion and out-portion each of size $\Theta\left(\lambda_{n} n^{2 / 3}\right)$.

The proof of this theorem will be given in Section 9. There we shall use the coupling of the trimmed out-graph $\widetilde{G}_{F_{n, p}}^{+}(x)$ to the random graph process $G_{n, 2 p-p^{2}}$ (see Lemma 2.6 and its proof) to explicitly construct $\Theta\left(\left|\lambda_{n}\right|^{3}\right)$ many hourglasses below threshold. To prove the theorem above threshold, we shall show that, when $\lambda$ is increased from its value below the threshold to its value above the threshold, a constant fraction of these subcritical hourglasses will merge into one giant hourglass of size $\Theta\left(\left|\lambda_{n}\right|^{3}\right) \Theta\left(n^{2 / 3} / \lambda_{n}^{2}\right)$. See Section 9 for details.

Here, we shall use the hourglasses to derive the upper bounds on satisfiability both to the left and to the right of the window.

Proof of upper bound of Theorem 3.4 in the subcritical regime. To get the bound on the left, we increase $p$ from $\left(1-t n^{-1 / 3}\right) / 2 n$ to $\left(1-(t / 2) n^{-1 / 3}\right) / 2 n$, with $\lambda_{0} \leq t \leq \varepsilon_{0} n^{1 / 3}$. For any pair of vertices, whether or not there was a directed edge between them before, afterwards the probability of finding such an edge is at least $(t / 4) n^{-4 / 3}$. For each hourglass, for each pair of literals $u$ and $v$ in the out-portion of the hourglass, if the clause $\bar{u} \vee \bar{v}$ appears, then we claim that the central vertex and the entire in-portion of the hourglass is afterwards part of the spine of the formula. Indeed, let $x$ be such a vertex. Then $x \rightsquigarrow u$ and $x \rightsquigarrow v$ since $u$ and $v$ are in the out-portion of the hourglass. But the appearance of the clause $\bar{u} \vee \bar{v}$ implies that $u \rightarrow \bar{v}$, so that we have $x \rightsquigarrow u \rightarrow \bar{v}$. Together with $x \rightsquigarrow v$, which is equivalent to $\bar{v} \rightsquigarrow \bar{x}$, we conclude that $x \rightsquigarrow \bar{x}$. The probability of the event that the clause $\bar{u} \vee \bar{v}$ appears is at least $\Theta\left(\left(n^{2 / 3} / t^{2}\right)^{2} t n^{-4 / 3}\right)=\Theta\left(1 / t^{3}\right)$. If furthermore a clause appears that contains two literals in the in-portion, then the formula is not satisfiable. These events are independent, so the probability that they both occur is at least $\Theta\left(1 / t^{6}\right)$. But since with high probability there are $\Theta\left(t^{3}\right)$ hourglasses, with probability at least $\Theta\left(1 / t^{3}\right)$ the formula becomes unsatisfiable. Setting $t=2\left|\lambda_{n}\right|$ gives the desired upper bound on the left.

Proof of upper bound of Theorem 3.4 in the supercritical regime. To get the bound on the right, we start with $p=\left(1+t n^{-1 / 3}\right) / 2 n$ (with $\lambda_{0} \leq t \leq \varepsilon_{0} n^{1 / 3}$ ), where the probability that there is no giant hourglass is at most $\exp \left(-\Theta\left(t^{3}\right)\right)$, and then increase it to $\left(1+2 t n^{-1 / 3}\right) / 2 n$. Any clauses of the form $(\bar{u} \vee \bar{v})$ where $u$ and $v$ are in the out-portion of the the giant hourglass beforehand will afterwards appear with probability at least $t n^{-4 / 3}$. This will cause the in-portion of the giant hourglass to become part of the spine of the formula, except with probability that can be bounded by $\left(1-t n^{-4 / 3}\right)^{\Theta\left(t n^{2 / 3}\right)^{2}}=\exp \left(-\Theta\left(t^{3}\right)\right)$. Furthermore, any clauses of the form $(u \vee v)$ where $u$ and $v$ are in the in-portion of the giant hourglass beforehand will afterwards appear with probability at least $t n^{-4 / 3}$. 
Therefore the formula will become unsatisfiable, except with probability that is again exponentially small in $t^{3}$. Setting $t=\lambda_{n} / 2$ completes the proof.

Remark 4.4. Instead of using Theorem 4.3, one can alternatively use Theorems 3.1 and 3.2 to prove that below the window, the spine has size at least $E\left(\left|S\left(F_{n, p}\right)\right|\right) / 2$ with probability $\exp (-O(1))$. Increasing $p$ as in the above proof, one obtains an alternative proof of the fact that, with probability at least $\Theta\left(1 /\left|\lambda_{n}\right|^{3}\right)$, the formula becomes unsatisfiable below the threshold. While a similar argument can be used to show that above the window, the probability of satisfiability goes to zero, we cannot use Theorems 3.1 and 3.2 alone to prove that it goes to zero exponentially fast in $\lambda_{n}^{3}$. For this, we need the hourglass theorem. 


\section{MACHINERY From RANDOM GRAPH TheORY}

In this section we establish several bounds needed in the proofs of Theorems 3.1 and 3.2. As in earlier sections, we use the notation $G_{n, p}$ for the (unoriented) random graph on $\{1,2, \ldots, n\}$ with edge probability $p$. We also consider $D_{n, p}$, the random directed graph on $\{1,2, \ldots, n\}$ in which each oriented edge is chosen independently with probability $p$. We use the symbol $L_{n, p}^{+}(x)$ to denote both the set of vertices $y \in\{1,2, \ldots, n\}$ that can be reached from a vertex $x$ in the random digraph $D_{n, p}$, and the set of vertices $y \in[n]$ that can be reached from a vertex $x$ in the digraph corresponding to a random 2-SAT formula $F_{n, p}$. If the difference is not clear from the context, we shall use the notations $L_{D_{n, p}}^{+}(x)$ and $L_{F_{n, p}}^{+}(x)$ to distinguish the two cases. We begin this section with a basic lemma which is implicit in the work of Karp [Kar90].

Lemma 5.1. The probability that in the random digraph $D_{n, p}$ every vertex can be reached from a given vertex is precisely the probability that the random graph $G_{n, p}$ is connected.

Proof. We may assume that the vertex in question is vertex 1. First, we shall inductively define a subtree $T=T\left(D_{n, p}\right)$ of $D_{n, p}$, rooted at 1 , with each edge oriented away from 1. To this end, set $X_{0}=\{1\}, Y_{0}=\emptyset$, and let $T_{0}$ be the subtree of $D_{n, p}$ with the single vertex 1. Suppose that we have defined a pair $\left(X_{i}, Y_{i}\right)$ of subsets of $\{1,2, \ldots, n\}$ with $Y_{i} \subset X_{i}$, and a subtree $T_{i}$ with $V\left(T_{i}\right)=X_{i}$. (We think of $Y_{i}$ as the set of vertices we have "exposed", i.e. tested for outgoing edges, and $X_{i}$ as the set of vertices we have selected so far.) If $X_{i}=Y_{i}$ then $T_{i}$ is our tree $T$. Otherwise, let $x_{i}$ be the smallest element of $X_{i} \backslash Y_{i}$. Let $\Gamma^{+}\left(x_{i}\right)$ denote the set of vertices in $\{1,2, \ldots, n\}$ that can be reached by single edges of $D_{n, p}$ that are oriented outward from $x_{i}$. Now set $X_{i+1}=X_{i} \cup \Gamma^{+}\left(x_{i}\right), Y_{i+1}=Y_{i} \cup\left\{x_{i}\right\}$, and take $T_{i+1}$ to be obtained from $T_{i}$ by adding to it the vertices in $X_{i+1} \backslash X_{i}$, together with all the edges from $x_{i}$ to $X_{i+1} \backslash X_{i}$. The vertex set of the subtree $T$ of $D_{n, p}$ constructed in this way is clearly $L_{n, p}^{+}(1)$; in particular, $L_{n, p}^{+}(1)=\{1,2, \ldots, n\}$ iff $V(T)=\{1,2, \ldots, n\}$. Since the edges of $T$ are oriented away from 1 , we may view $T$ as an unoriented tree.

Now, let us construct a subtree $T^{\prime}=T^{\prime}\left(G_{n, p}\right)$ of the random graph $G_{n, p}$ rooted at 1 by precisely the same algorithm. The lemma will follow if we show that

$$
\mathbb{P}\left(T\left(D_{n, p}\right)=T_{0}\right)=\mathbb{P}\left(T^{\prime}\left(G_{n, p}\right)=T_{0}\right)
$$

for every tree $T_{0}$ with vertex set $\{1,2, \ldots, n\}$.

Given $T_{0}$, we can define the $X_{i}$ 's as above. We have $T\left(D_{n, p}\right)=T_{0}$ if and only if the random digraph $D_{n, p}$ is such that

1) it contains all the edges of $T_{0}$ (oriented away from vertex 1 ),

2) it contains no edge oriented from $x_{i}$ to $\{1,2, \ldots, n\} \backslash X_{i+1}$.

Similarly, $T^{\prime}\left(G_{n, p}\right)=T_{0}$ if and only if the random graph $G_{n, p}$ is such that

1) it contains all the edges of $T_{0}$,

2) it contains no edge from $x_{i}$ to $\{1,2, \ldots, n\} \backslash X_{i+1}$.

Notice that the probability that $D_{n, p}$ contains a given set $K$ of oriented edges, and no edge of a second set $K^{\prime}$ of oriented edges, is $p^{|K|}(1-p)^{\left|K^{\prime}\right|}$ provided that $K \cap K^{\prime}=\emptyset$. Moreover, this is equal to the probability that $G_{n, p}$ contains a set $\widetilde{K}$ of unoriented edges, 
and no edge of a second set $\widetilde{K}^{\prime}$ of unoriented edges, provided that $\widetilde{K} \cap \widetilde{K}^{\prime}=\emptyset,|\widetilde{K}|=|K|$ and $\left|\widetilde{K}^{\prime}\right|=\left|K^{\prime}\right|$. Thus relation (5.1) holds, and we are done.

Returning to the 2-SAT problem $F_{n, p}$, let $x$ be a fixed literal. The probability that, in a random 2-SAT formula $F_{n, p}$, the set $L_{n, p}^{+}(x)$ consists of $k$ strictly distinct literals is trivially independent of $x$; we shall denote it by $P_{n, p}(k)$ :

$$
P_{n, p}(k)=\mathbb{P}\left(\left\{\left|L_{n, p}^{+}(x)\right|=k\right\} \cap\left\{L_{n, p}^{+}(x) \text { is s.d. }\right\}\right) .
$$

Lemma 5.2. For all $n, k$ and $p$, with $1 \leq k \leq n$ and $0<p<1$, we have

$$
P_{n, p}(k)=2^{k-1}\left(\begin{array}{c}
n-1 \\
k-1
\end{array}\right)(1-p)^{2 k n-3 k^{2} / 2-k / 2} \mathbb{P}\left(G_{k, p} \text { is connected }\right) .
$$

Proof. Let $X$ be a set of $k$ strictly distinct literals with $x \in X$. For $y, z \in X$, the dual of the implication $y \rightarrow z$ involves no literal in $X$. Therefore the probability that $L_{F_{n, p}}^{+}(x)=X$ is $P_{a} P_{b}$, where $P_{a}$ is the probability that every vertex of the random digraph $D_{X, p}$ can be reached from $x$ and $P_{b}$ is the probability that the random 2-SAT formula $F_{n, p}$ contains no implication from the set $I\left(X, X^{c}\right)=\{y \rightarrow z: y \in X, z \notin X\}$.

By Lemma 5.1, we have $P_{a}=\mathbb{P}\left(G_{k, p}\right.$ is connected $)$, so we turn to the task of calculating the probability $P_{b}$.

Note that there are $k(2 n-k)$ implications in the set $I\left(X, X^{c}\right)$. However, a 2-SAT formula $F_{n, p}$ contains none of the $k$ implications $y \rightarrow \bar{y}, y \in X$. Also, if $y \in X$ and $z \in \bar{X}$, then $y \rightarrow z$ and $\bar{z} \rightarrow \bar{y}$ are dual implications, i.e. $F_{n, p}$ contains $y \rightarrow z$ if and only if it contains $\bar{z} \rightarrow \bar{y}$. In fact, both implications $y \rightarrow z$ and $\bar{z} \rightarrow \bar{y}$ belong to $I\left(X, X^{c}\right)$ if and only if $y \in X, z \in \bar{X}$ and $y \neq \bar{z}$. Hence $I\left(X, X^{c}\right)$ contains $\left(k^{2}-k\right) / 2$ dual pairs, so that the probability that $F_{n, p}$ contains no implication from $I\left(X, X^{c}\right)$ is

$$
P_{b}=(1-p)^{k(2 n-k)-k-\left(k^{2}-k\right) / 2}=(1-p)^{2 k n-3 k^{2} / 2-k / 2} .
$$

Therefore,

$$
\mathbb{P}\left(L_{F_{n, p}}^{+}(x)=X\right)=\mathbb{P}\left(G_{k, p} \text { is connected }\right)(1-p)^{2 k n-3 k^{2} / 2-k / 2} .
$$

Since there are $2^{k-1}\left(\begin{array}{l}n-1 \\ k-1\end{array}\right)$ choices for the set $X$, the lemma is proved.

In order to transform Lemma 5.2 into a form suitable for applications, note that the probability that $G_{k, p}$ is connected is trivially expressed in terms of $f(k, m)$, the number of connected labelled graphs with $k$ vertices and $m$ edges, and that $f(k, m)$ has a good and fairly simple approximation when $m-k$ is not too large compared to $k$. To state this approximation, let us define an array of numbers $c_{k, \ell}$ by $f(k, k-1+\ell)=c_{k, \ell} k^{k-2+3 \ell / 2}$. The somewhat peculiar choice of the parameter $\ell$ is justified by the fact that $c_{k, \ell} \neq 0$ if and only if $0 \leq \ell \leq\left(\begin{array}{l}k \\ 2\end{array}\right)-k+1$. Also, if $\ell$ is not too large then $f(k, k-1+\ell)$ has order about $k^{k-2+3 \ell / 2}$. More precisely, since $f(k, k-1)$ is just the number of trees with $k$ labelled vertices, by Cayley's theorem we have $c_{k, 0}=1$. Also,

$$
c_{k, 1}=\left(1+O\left(k^{-1 / 2}\right)\right)(\pi / 8)^{1 / 2},
$$


$c_{k, \ell} \leq 1$ for all $k \geq 1$ and $\ell \geq 0$, and for all $2 \leq \ell \leq\left(\begin{array}{l}k \\ 2\end{array}\right)-k+1$ and some $c<\infty$, we have

$$
c_{k, \ell} \leq(c / \ell)^{\ell / 2}
$$

see Bol85]. When $\ell$ is fairly small compared to $k$, there are rather detailed estimates for $c_{k, \ell}$. To be precise, Wright (Wri77, Wri80) showed that for $2 \leq \ell=o\left(k^{1 / 3}\right)$ we have

$$
c_{k, \ell}=\gamma(3 \pi)^{1 / 2}\left(\frac{e}{12(\ell-1)}\right)^{(\ell-1) / 2}\left(1+o_{\ell}(1)\right),
$$

where $\gamma=0.159155 \ldots$ is the the limit of a certain bounded increasing sequence. Later Meertens proved that $\gamma=1 /(2 \pi)$ (see [BCM90]).

The probability that $G_{k, p}$ is connected and has $k-1+\ell$ edges is just

$$
c_{k, \ell} k^{k-2+3 \ell / 2} p^{k-1+\ell}(1-p)^{\left(\begin{array}{l}
k \\
2
\end{array}\right)-k+1-\ell},
$$

so Lemma 5.2 has the following immediate consequence.

Corollary 5.3. For all $n, k$ and $p$, with $1 \leq k \leq n$ and $0<p<1$, we have

$$
P_{n, p}(k)=\frac{1}{n}\left(\begin{array}{l}
n \\
k
\end{array}\right)(2 p k)^{k-1}(1-p)^{2 k n-k^{2}-2 k+1} S_{p}(k),
$$

where

$$
S_{p}(k)=\sum_{\ell=0}^{\left(\begin{array}{c}
k \\
2
\end{array}\right)-k+1} c_{k, \ell}\left(\frac{k^{3 / 2} p}{1-p}\right)^{\ell}
$$

If $k^{3 / 2} p /(1-p)$ is bounded, then

$$
S_{p}(k)=1+\sqrt{\frac{\pi}{8}} \frac{k^{3 / 2} p}{1-p}\left[1+O\left(k^{-1 / 2}\right)+\mathbb{O}_{0}\left(\frac{k^{3 / 2} p}{1-p}\right)\right],
$$

where $\mathbb{O}_{0}(\cdot)$ is the Landau symbol introduced at the beginning of Section 8 .

To estimate $P_{n, p}(k)$, we relate it to known bounds on related events in random graphs. To this end, we recall the definition of the trimmed out-graph $\widetilde{D}_{F}^{+}(x)$ in Section 2 and its relation to the random graph $G_{n, 2 p-p^{2}}$ on $n$ vertices with edge probability $2 p-p^{2}$, see Lemma 2.6. Recall that the vertex set of $\widetilde{D}_{F_{n, p}}^{+}(x)$ is denoted by $\widetilde{L}_{n, p}^{+}(x)$. We define

$$
Q_{n, p}(k)=\mathbb{P}\left(\left|\widetilde{L}_{n, p}^{+}(x)\right|=k\right) .
$$

By Lemma 2.6 part (iv),

$$
Q_{n, p}(k)=\mathbb{P}\left\{\left|C_{n, 2 p-p^{2}}(x)\right|=k\right\},
$$


where $C_{n, 2 p-p^{2}}(x)$ is the connected component in $G_{n, 2 p-p^{2}}$ containing a fixed vertex $x$. For all $n, k$ and $p$, with $1 \leq k \leq n$ and $0<p<1$, we have

$$
\begin{aligned}
Q_{n, p}(k) & =\left(\begin{array}{l}
n-1 \\
k-1
\end{array}\right)\left(1-2 p+p^{2}\right)^{k(n-k)} \mathbb{P}\left(G_{k, 2 p-p^{2}} \text { is connected }\right) \\
& =\frac{1}{n}\left(\begin{array}{l}
n \\
k
\end{array}\right)\left(\left(2 p-p^{2}\right) k\right)^{k-1}(1-p)^{2 k n-2 k^{2}+k^{2}-3 k+2} S_{2 p-p^{2}}(k) .
\end{aligned}
$$

In Section 9, we shall also need bounds on the probability $R_{n, p}(k)$ that $C_{n, 2 p-p^{2}}(x)$ is a tree of size $k$,

$$
R_{n, p}(k)=\mathbb{P}\left(\left|C_{n, 2 p-p^{2}}(x)\right|=k \text { and } C_{n, 2 p-p^{2}}(x) \text { is a tree }\right) .
$$

Recalling the derivation of Corollary 5.3, we immediately see that $Q_{n, p}(k)$ and $R_{n, p}(k)$ are related by

$$
Q_{n, p}(k)=R_{n, p}(k) S_{2 p-p^{2}}(k) .
$$

The following lemma will be used to turn well-known bounds on $Q_{n, p}(k)$ into bounds on $P_{n, p}(k)$.

Lemma 5.4. For all $0<p<1$,

$$
P_{n, p}(k) \leq Q_{n, p}(k)
$$

If $p \leq 1 / 2$ and $k^{3 / 2} p$ is bounded, then

$$
0 \leq Q_{n, p}(k)-P_{n, p}(k)=\sqrt{\frac{\pi}{8}} \frac{k^{3 / 2} p}{1-p}\left[1+O\left(k^{-1 / 2}\right)+\mathbb{O}_{0}\left(\frac{k^{3 / 2} p}{1-p}\right)\right] P_{n, p}(k) .
$$

If $p \leq 1 / 2$ and $k^{3 / 2} p \geq 1$, then

$$
P_{n, p}(k)=O\left(\ell_{0} 2^{-\ell_{0}} Q_{n, p}(k)\right)
$$

where

$$
\ell_{0}=\ell_{0}(k)=\min \left\{\frac{k^{3} p^{2}}{12(1-p)^{2}}, n^{1 / 5}\right\}
$$

Proof. By Lemma 2.6, $L_{F_{n, p}}^{+}(x)$ is strictly distinct if and only if $L_{F_{n, p}}^{+}(x)=\widetilde{L}_{F_{n, p}}^{+}(x)$. As a consequence,

$$
\begin{aligned}
P_{n, p}(k) & =\mathbb{P}\left(L_{F_{n, p}}^{+}(x)=\widetilde{L}_{F_{n, p}}^{+}(x) \text { and }\left|L_{F_{n, p}}^{+}(x)\right|=k\right) \\
& =\mathbb{P}\left(L_{F_{n, p}}^{+}(x)=\widetilde{L}_{F_{n, p}}^{+}(x) \text { and }\left|\widetilde{L}_{F_{n, p}}^{+}(x)\right|=k\right) \\
& \leq \mathbb{P}\left(\left|\widetilde{L}_{F_{n, p}}^{+}(x)\right|=k\right)=Q_{n, p}(k),
\end{aligned}
$$


which proves (5.16). Rewriting (5.13) in the form

$$
\begin{aligned}
Q_{n, p}(k) & =\frac{1}{n}\left(\begin{array}{l}
n \\
k
\end{array}\right)(2 p k)^{k-1}(1-p / 2)^{k-1}(1-p)^{2 k n-k^{2}-3 k+2} S_{2 p-p^{2}}(k) \\
& =P_{n, p}(k)(1-p / 2)^{k-1}(1-p)^{-k+1} S_{2 p-p^{2}}(k) / S_{p}(k) \\
& \geq P_{n, p}(k) S_{2 p-p^{2}}(k) / S_{p}(k)
\end{aligned}
$$

and observing that $S_{p}(k)$ is a monotone increasing function of $p /(1-p)$, and hence of $p$ (see (5.9)), we have $S_{2 p-p^{2}}(k) \geq S_{p}(k)$, obtaining an alternative proof of the bound (5.16).

If $p \leq 1 / 2$ and $k^{3 / 2} p$ is bounded, then both $k^{3 / 2} p /(1-p)$ and $k^{3 / 2}\left(2 p-p^{2}\right) /\left(1-2 p+p^{2}\right)$ are bounded by a constant times $k^{3 / 2} p$. By (5.10), we therefore have

$$
\frac{S_{2 p-p^{2}}(k)}{S_{p}(k)}=1+\sqrt{\frac{\pi}{8}} \frac{k^{3 / 2} p}{1-p}\left[1+O\left(k^{-1 / 2}\right)+\mathbb{O}_{0}\left(\frac{k^{3 / 2} p}{1-p}\right)\right]
$$

and hence by (5.21)

$$
Q_{n, p}(k)=P_{n, p}(k)(1+O(k p))\left(1+\sqrt{\frac{\pi}{8}} \frac{k^{3 / 2} p}{1-p}\left[1+O\left(k^{-1 / 2}\right)+\mathbb{O}_{0}\left(\frac{k^{3 / 2} p}{1-p}\right)\right]\right) .
$$

As a consequence,

$$
\frac{Q_{n, p}(k)}{P_{n, p}(k)}-1=\sqrt{\frac{\pi}{8}} \frac{k^{3 / 2} p}{1-p}\left[1+O\left(k^{-1 / 2}\right)+\mathbb{O}_{0}\left(\frac{k^{3 / 2} p}{1-p}\right)\right]
$$

which implies the bound (5.17).

In order to prove (5.18), we decompose $S_{p}(k)$ as

$$
\begin{aligned}
S_{p}(k)=\sum_{\ell \geq 0} c_{k, \ell}\left(\frac{k^{3 / 2} p}{1-p}\right)^{\ell} & =\sum_{0 \leq \ell<\ell_{0}} c_{k, \ell}\left(\frac{k^{3 / 2} p}{1-p}\right)^{\ell}+\sum_{\ell \geq \ell_{0}} c_{k, \ell}\left(\frac{k^{3 / 2} p}{1-p}\right)^{\ell} \\
& =S_{p}^{\prime}(k)+S_{p}^{\prime \prime}(k),
\end{aligned}
$$

where $S_{p}^{\prime}(k)$ is the first and $S_{p}^{\prime \prime}(k)$ is the second sum above. If $\ell \leq n^{1 / 5}$ then $\ell=o\left(k^{1 / 3}\right)$, so by $(5.7)$ we have

$$
S_{p}^{\prime}(k)=O\left(\sum_{2 \leq \ell<\ell_{0}}\left(\frac{e^{1 / 2} k^{3 / 2} p}{12^{1 / 2}(\ell-1)^{1 / 2}(1-p)}\right)^{(\ell-1)} k^{3 / 2} p\right) .
$$

By taking the ratio of successive terms in the series

$$
\begin{aligned}
\left(\frac{e^{1 / 2} k^{3 / 2} p}{12^{1 / 2}(\ell-1)^{1 / 2}(1-p)}\right)^{-(\ell-1)}\left(\frac{e^{1 / 2} k^{3 / 2} p}{12^{1 / 2} \ell^{1 / 2}(1-p)}\right)^{\ell} & =\left(\frac{e^{1 / 2} k^{3 / 2} p}{12^{1 / 2}(1-p)}\right) \frac{1}{\ell^{1 / 2}}\left(1+\frac{1}{\ell-1}\right)^{-(\ell-1) / 2} \\
& \geq\left(\frac{e^{1 / 2} k^{3 / 2} p}{12^{1 / 2}(1-p)}\right) \frac{1}{\ell^{1 / 2}} e^{-1 / 2}
\end{aligned}
$$

we see that the summand is an increasing function of $\ell$ for $2 \leq \ell \leq \ell_{0}+1$. Since the $\left(\ell_{0}+1\right)$ st term is in the sum for $S_{p}^{\prime \prime}(k)$, we then have

$$
S_{p}^{\prime}(k)=O\left(\ell_{0} S_{p}^{\prime \prime}(k)\right) \text {. }
$$


To bound $S_{p}^{\prime \prime}(k)$, we note that

$$
\frac{2 p}{1-p}=\frac{2 p-p^{2}}{1-\left(2 p-p^{2}\right)}
$$

so that

$$
\begin{aligned}
S_{p}^{\prime \prime}(k) & =\sum_{\ell \geq \ell_{0}} c_{k, \ell}\left(\frac{k^{3 / 2} p}{1-p}\right)^{\ell} \\
& \leq 2^{-\ell_{0}} \sum_{\ell \geq \ell_{0}} c_{k, \ell}\left(\frac{k^{3 / 2} 2 p}{1-p}\right)^{\ell} \\
& =2^{-\ell_{0}} \sum_{\ell \geq \ell_{0}} c_{k, \ell}\left(\frac{k^{3 / 2}\left(2 p-p^{2}\right)}{1-\left(2 p-p^{2}\right)}\right)^{\ell} \\
& =2^{-\ell_{0}} S_{2 p-p^{2}}^{\prime \prime}(k)
\end{aligned}
$$

and therefore

$$
S_{p}(k)=O\left(\ell_{0} 2^{-\ell_{0}} S_{2 p-p^{2}}^{\prime \prime}(k)\right)=O\left(\ell_{0} 2^{-\ell_{0}} S_{2 p-p^{2}}(k)\right) .
$$

Combined with (5.22), this gives the desired bound (5.18).

Our later estimates rely heavily on bounds on the expectation of the size of the component of a given vertex in a random graph, Lemma 5.5 below. Although sharper forms of these bounds were already proved by Bollobás [Bol84, Euczak [Euc90], and Janson et al. JKŁP94, these previous estimates were proved only for a restricted range of $\lambda$ (e.g. $\lambda \leq n^{1 / 12}$ ), whereas we require the full range (i.e. $\lambda_{n} \leq \varepsilon_{0} n^{1 / 3}$ ). These estimates turn out to be rather involved; the proof is given in Appendix $\mathrm{B}$.

We remark that the bound we shall obtain in (5.24) below is closely related to the order of the giant component: if $\lambda_{n} \rightarrow \infty$ and $\lambda_{n}=o\left(n^{1 / 3}\right)$ then, for $p=\left(1+\lambda_{n} n^{-1 / 3}\right) / n$, with probability tending to 1 , the random graph $G_{n, p}$ has a unique giant component with $(2+o(1)) \lambda_{n} n^{2 / 3}$ vertices.

Lemma 5.5. There are constants $c, \varepsilon_{0}$ and $\lambda_{0}, c>0,0<\varepsilon_{0}<1$, and $0<\lambda_{0}<\infty$ with the following property. Let $\lambda_{0} \leq \lambda_{n} \leq \varepsilon_{0} n^{1 / 3}$ and, as before, set $\varepsilon=\lambda_{n} n^{-1 / 3}$ and $p=\left(1+\lambda_{n} n^{-1 / 3}\right) / 2 n$. Then

$$
\sum_{k \geq \lambda_{n} n^{2 / 3}} Q_{n, p}(k)=\vartheta(\varepsilon)\left(1+O\left(1 / \lambda_{n}^{2}\right)\right)
$$

and

$$
\sum_{k=\left\lfloor n^{2 / 3} / \lambda_{n}\right\rfloor}^{\left\lceil\lambda_{n} n^{2 / 3}\right\rceil} Q_{n, p}(k)=O\left(e^{-c \lambda_{n}} n^{-1 / 3}\right) .
$$




\section{Moment Estimates}

In this section we shall bound the moments of the number of literals $x$ for which the set $L_{n, p}^{+}(x)$ consists of strictly distinct literals. Recall that $P_{n, p}(k)$ is the probability that, for a variable $x$, the set $L_{n, p}^{+}(x)$ consists of $k$ strictly distinct literals. We use Corollary 5.3 to get a good estimate of $P_{n, p}(k)$. Note that

$$
\begin{aligned}
\frac{1}{n}\left(\begin{array}{l}
n \\
k
\end{array}\right)(2 p k)^{k-1} & =\frac{1}{2 p n k} \frac{(k / e)^{k}}{k !}(2 n p e)^{k} \prod_{i=0}^{k-1}\left(1-\frac{i}{n}\right) \\
& =\frac{\exp \left[-1 /\left(12 k+\delta_{k}\right)\right]}{2 p n \sqrt{2 \pi} k^{3 / 2}}(2 n p e)^{k} \prod_{i=0}^{k-1}\left(1-\frac{i}{n}\right)
\end{aligned}
$$

where $0 \leq \delta_{k} \leq 1$ for each $k$. Recalling that $2 n p=1+\varepsilon=1+\lambda_{n} n^{-1 / 3}$, with $|\varepsilon| \leq \varepsilon_{0}$, $\varepsilon_{0}<1$, we write

$$
\log (1+\varepsilon)=\varepsilon-\frac{1}{2} \varepsilon^{2}+\Theta\left(\varepsilon^{3}\right)
$$

and

$$
\log \left(1-\frac{i}{n}\right)=-\frac{i}{n}-\frac{i^{2}}{2 n^{2}}-\Theta\left(\frac{i^{3}}{n^{3}}\right)
$$

and obtain

$$
\begin{aligned}
\frac{1}{n}\left(\begin{array}{l}
n \\
k
\end{array}\right)(2 p k)^{k-1}=\frac{\exp [-\Theta(1 / k)]}{2 p n \sqrt{2 \pi} k^{3 / 2}} & \exp \left\{k+k \varepsilon-\frac{1}{2} k \varepsilon^{2}+k \Theta\left(\varepsilon^{3}\right)\right\} \\
\times & \exp \left\{-\frac{k^{2}}{2 n}+\Theta\left(\frac{k}{n}\right)-\frac{k^{3}}{6 n^{2}}+\Theta\left(\frac{k^{2}}{n^{2}}\right)-\Theta\left(\frac{k^{4}}{n^{3}}\right)\right\} .
\end{aligned}
$$

Expressing

$$
\begin{aligned}
(1-p)^{2 k n-k^{2}-2 k+1} & =\exp \left\{-\left(p+\Theta\left(p^{2}\right)\right)\left(2 k n-k^{2}-2 k+1\right)\right\} \\
& =\exp \left\{-k-\varepsilon k+\frac{k^{2}}{2 n}+\frac{\varepsilon k^{2}}{2 n}+\frac{k(1+\varepsilon)}{n}-\Theta\left(p^{2} k n\right)\right\},
\end{aligned}
$$

we therefore get

$$
\begin{aligned}
P_{n, p}(k)= & \frac{1}{2 p n \sqrt{2 \pi} k^{3 / 2}} \times \\
& \exp \left\{-\frac{k \varepsilon^{2}}{2}-\frac{k^{3}}{6 n^{2}}+\frac{\varepsilon k^{2}}{2 n}+O\left(\frac{k}{n}\right)-\Theta\left(\frac{k^{4}}{n^{3}}\right)+k \Theta\left(\varepsilon^{3}\right)-\Theta(1 / k)\right\} S_{p}(k) .
\end{aligned}
$$


Lemma 6.1. If $k / n^{2 / 3}$ is bounded, then $P_{n, p}(k), Q_{n, p}(k)$ and $R_{n, p}(k)$ can be rewritten in the form

$$
\frac{1}{2 p n \sqrt{2 \pi} k^{3 / 2}} \exp \left\{-\frac{k \varepsilon^{2}}{2}\left(1-\Theta(\varepsilon)-\Theta\left(\frac{k n^{-2 / 3}}{\lambda_{n}}\right)\right)+\mathbb{O}_{0}\left(\frac{k^{3 / 2}}{n}\right)-\Theta(1 / k)\right\} .
$$

Remark 6.2. It is evident that the multiplicative error terms $1-\Theta(\varepsilon)-\Theta\left(k n^{-2 / 3} / \lambda_{n}\right)$ above can be made arbitrarily close to 1 if $\varepsilon$ is small enough and $\lambda_{n}$ is large enough, i.e. if $1 \ll \lambda_{n} \ll n^{1 / 3}$, or equivalently $n^{-1 / 3} \ll \varepsilon \ll 1$. Likewise the additive error terms can be made arbitrarily small when $1 \ll k \ll n^{2 / 3}$. We follow the standard convention that "if $a \ll b$ then $c=(1+o(1)) d$ " means that the $o(1)$ error term can be made arbitrarily small by taking the ratio $b / a$ large enough, and in our earlier notation may be re-expressed as "c $=\left(1+o_{b / a}(1)\right) d . "$

Proof of Lemma 6.1. With the assumption that $k / n^{2 / 3}$ is bounded, we can use our bound (5.10) on $S_{p}(k)$ together with the bounds $k / n \leq k^{3 / 2} / n, k^{4} / n^{3}=O\left(k^{3} / n^{2}\right)=\mathbb{O}_{0}\left(k^{3 / 2} / n\right)$, and $k^{3} / n^{2}=\mathbb{O}_{0}\left(k^{3 / 2} / n\right)$ to rewrite 6.1$)$ as

$$
P_{n, p}(k)=\frac{1}{2 p n \sqrt{2 \pi} k^{3 / 2}} \exp \left\{-\frac{k \varepsilon^{2}}{2}(1-\Theta(\varepsilon))+\frac{\varepsilon k^{2}}{2 n}+\mathbb{O}_{0}\left(\frac{k^{3 / 2}}{n}\right)-\Theta(1 / k)\right\} .
$$

Note that the ratio between the second and first terms in the exponential is $k /(n \varepsilon)=$ $k /\left(\lambda_{n} n^{2 / 3}\right)$, so we can further rewrite (6.3) as (6.2). The estimate (6.2) for $Q_{n, p}(k)$ follows immediately from the estimate for $P_{n, p}(k)$ and (5.23), and the estimate for $R_{n, p}(k)$ follows from that for $Q_{n, p}(k)$ and (5.15).

Lemma 6.3. There are constants $c, \varepsilon_{0}$ and $\lambda_{0}$ with $c>0,0<\varepsilon_{0}<1$ and $0<\lambda_{0}<\infty$, such that the following statements hold for $a>1 / 2$ and $\lambda_{0} \leq|\lambda| \leq \varepsilon_{0} n^{1 / 3}$.

i) If $\lambda_{n}<0$, then

$$
\sum_{k \geq n^{2 / 3} /\left|\lambda_{n}\right|} k^{a} Q_{n, p}(k)=O\left(\left[\frac{2}{\varepsilon^{2}}\right]^{a-1 / 2} e^{-c\left|\lambda_{n}\right|}\right),
$$

where the constant implicit in the Landau symbol $O(\cdot)$ depends on a.

ii) For both positive and negative $\lambda_{n}$, we have

$$
\sum_{k \leq n^{2 / 3} /\left|\lambda_{n}\right|} k^{a} Q_{n, p}(k)=\frac{1+o_{\varepsilon, \lambda_{n}}(1)}{2 p n} \frac{\Gamma(a-1 / 2)}{\sqrt{2 \pi}}\left[\frac{2}{\varepsilon^{2}}\right]^{a-1 / 2},
$$

where the $o_{\varepsilon, \lambda_{n}}(1)$ term depends upon a, and for fixed a becomes as small as we like if $\left|\lambda_{n}\right|$ is large enough and $\varepsilon=\lambda_{n} / n^{1 / 3}$ is small enough.

Remark 6.4. Here, as in the rest of this paper, $\Gamma$ denotes the gamma function, which interpolates factorials. Recalling that $\Gamma(1 / 2)=\sqrt{\pi}$, the lemma immediately implies that 
below the window the expected size a component of a given vertex in $G_{n, 2 p-p^{2}}$ can be estimated by

$$
E\left(\left|C_{n, 2 p-p^{2}}(x)\right|\right)=\frac{1}{2 p n|\varepsilon|}\left(1+o_{\varepsilon, \lambda_{n}}(1)\right) .
$$

Note also that (6.4) implies that below the window

$$
\sum_{k \geq n^{2 / 3} /\left|\lambda_{n}\right|} Q_{n, p}(k)=\sum_{k \geq n^{2 / 3} /\left|\lambda_{n}\right|} \frac{k}{k} Q_{n, p}(k) \leq \frac{\lambda_{n}}{n^{2 / 3}} \sum_{k \geq n^{2 / 3} /\left|\lambda_{n}\right|} k Q_{n, p}(k)=O\left(n^{-1 / 3} e^{-c\left|\lambda_{n}\right|}\right) .
$$

Proof of Lemma 6.3. We start with the proof of ii). The limit of the summation is $n^{2 / 3} /|\lambda|=n^{1 / 3} /|\varepsilon| ;$ we first sum the portion up to $1 / \varepsilon$ separately. For this portion, the sum is

$$
\sum_{k<1 /|\varepsilon|} k^{a} Q_{n, p}(k)=O\left((1 / \varepsilon)^{a-1 / 2}\right)
$$

where here and throughout this proof, constants may depend upon $a$.

Referring to our expression (6.2) for $Q_{n, p}(k)$, we see that for the remainder of the terms in the sum, the additive error terms in the exponential tend to zero when $k / n^{2 / 3} \leq 1 /|\lambda|$ is small enough and $k \geq 1 /|\varepsilon|$ is large enough, and so they contribute only a multiplicative error of $1+o(1)$. The multiplicative error terms in the exponential can also be made arbitrarily close to 1 by taking $|\lambda|$ large enough and $|\varepsilon|$ small enough. Therefore we have

$$
\frac{1+o_{\varepsilon, \lambda_{n}}(1)}{2 p n \sqrt{2 \pi}} B(2-\delta) \leq \sum_{1 /|\varepsilon| \leq k \leq n^{1 / 3} /|\varepsilon|} k^{a} Q_{n, p}(k) \leq \frac{1+o_{\varepsilon, \lambda_{n}}(1)}{2 p n \sqrt{2 \pi}} B(2+\delta)
$$

where $\delta=o_{\varepsilon, \lambda_{n}}(1)$ and

$$
B(t)=\sum_{1 /|\varepsilon| \leq k \leq n^{1 / 3} /|\varepsilon|} k^{a-3 / 2} \exp \left[-\frac{k \varepsilon^{2}}{t}\right]
$$


Since the summand in (6.10) is unimodal, we may approximate the sum with an integral

$$
\begin{aligned}
B(t) & =\int_{1 /|\varepsilon|}^{n^{1 / 3} /|\varepsilon|} k^{a-3 / 2} \exp \left[-\frac{k \varepsilon^{2}}{t}\right] d k+O\left(\max k^{a-3 / 2} \exp \left[-\frac{k \varepsilon^{2}}{t}\right]\right) \\
& =\int_{|\varepsilon| / t}^{n^{1 / 3}|\varepsilon| / t}\left[\frac{t u}{\varepsilon^{2}}\right]^{a-3 / 2} \exp [-u] \frac{t d u}{\varepsilon^{2}}+O\left(\left[\frac{1}{\varepsilon^{2}}\right]^{a-3 / 2}\right) \\
& =\left[\frac{t}{\varepsilon^{2}}\right]^{a-1 / 2} \int_{|\varepsilon| / t}^{|\lambda| / t} u^{a-3 / 2} \exp [-u] d u+O\left(\left[\frac{1}{\varepsilon^{2}}\right]^{a-3 / 2}\right) \\
& =\left[\frac{t}{\varepsilon^{2}}\right]^{a-1 / 2}\left[\Gamma(a-1 / 2)+o_{\varepsilon, \lambda_{n}}(1)\right]+O\left(\left[\frac{1}{\varepsilon^{2}}\right]^{a-3 / 2}\right) \\
& =\left[\frac{t}{\varepsilon^{2}}\right]^{a-1 / 2}\left[\Gamma(a-1 / 2)+o_{\varepsilon, \lambda_{n}}(1)\right]
\end{aligned}
$$

where the $o_{\varepsilon, \lambda_{n}}(1)$ depends on $a$. Combining (6.8), (6.9) and (6.11), we get (6.5).

To prove i), we use the well know fact that the cluster size distribution in $G_{n, \widetilde{p}}$, with $\widetilde{p}=2 p-p^{2}$, is stochastically dominated by a birth process with binominal offspring distribution $\operatorname{Binomial}(n, \widetilde{p})$, which in turn is stochastically dominated by a Poisson birth process with parameter $n \log [1 /(1-\widetilde{p})]=2 n p(1+O(1 / n))$. Writing this parameter as $1+\widetilde{\varepsilon}$, we therefore get the estimate

$$
\begin{aligned}
\sum_{k>\frac{n^{2} / 3}{\left|\lambda_{n}\right|}} k^{a} Q_{n, p}(k) & \leq \frac{1}{1+\widetilde{\varepsilon}} \sum_{k \geq n^{2 / 3} /\left|\lambda_{n}\right|} k^{a} \frac{k^{k-1}}{k !}\left((1+\widetilde{\varepsilon}) e^{-(1+\widetilde{\varepsilon})}\right)^{k} \\
& \leq \frac{1}{1+\widetilde{\varepsilon}} \sum_{k \geq n^{2 / 3} /\left|\lambda_{n}\right|}^{n} \frac{k^{a}}{\sqrt{2 \pi k^{3}}} \exp \left(-\frac{\widetilde{\varepsilon}^{2}}{2} k\right) \\
& =\frac{1+O(1 / n)}{2 p n \sqrt{2 \pi}} \sum_{k \geq \frac{n^{2} / 3}{|\lambda|}} k^{a-3 / 2} \exp \left\{-\frac{k \varepsilon^{2}}{2}\left(1+o_{n}(1)\right)\right\} .
\end{aligned}
$$

Bounding the sum on the right hand side by $O\left(\left[\left(n^{2 / 3}\right) \lambda_{n}^{-2}\right]^{a-1 / 2} e^{-c\left|\lambda_{n}\right|}\right)$, we obtain the estimate (6.4).

Lemma 6.5. There are constants $c, \varepsilon_{0}$ and $\lambda_{0}$ with $c>0,0<\varepsilon_{0}<1$ and $0<\lambda_{0}<\infty$, such that for $a>1 / 2$ and $\lambda_{0} \leq|\lambda| \leq \varepsilon_{0} n^{1 / 3}$, we have

$$
\sum_{k=1}^{n} k^{a} P_{n, p}(k)=\frac{1+o_{\varepsilon, \lambda_{n}}(1)}{2 p n} \frac{\Gamma(a-1 / 2)}{\sqrt{2 \pi}}\left[\frac{2}{\varepsilon^{2}}\right]^{a-1 / 2}
$$


and

$$
\sum_{k \geq n^{2 / 3} /\left|\lambda_{n}\right|} k^{a} P_{n, p}(k)=\left[\frac{1}{\varepsilon^{2}}\right]^{a-1 / 2}\left\{\begin{array}{lll}
O\left(e^{-\Theta\left(\left|\lambda_{n}\right|\right)}\right) & \text { if } & \lambda_{n}<0 \\
O\left(e^{-\Theta\left(\lambda_{n}^{-3 / 5}\right)}\right) & \text { if } & \lambda_{n}>0
\end{array}\right.
$$

where the constants implicit in both the $o_{\varepsilon, \lambda_{n}}(1)$ term and the $O$ term depends upon a, and where $\varepsilon=\lambda_{n} n^{-1 / 3}$ as usual.

Remark 6.6. The particular values of $a$ that we shall need are $a=1, a=3 / 2$, and $a=2$. For these values, we have

$$
\sum_{k} k^{a} P_{n, p}(k)=\frac{1+o_{\varepsilon, \lambda_{n}}(1)}{2 p n} \times \begin{cases}|\varepsilon|^{-1} & a=1 \\ \sqrt{2 / \pi} \varepsilon^{-2} & a=3 / 2 \\ |\varepsilon|^{-3} & a=2\end{cases}
$$

where as before the summation can range over all $k$, or over all $k \leq n^{2 / 3} /\left|\lambda_{n}\right|$.

Remark 6.7. Using the same trick as in (6.7), we can write

$$
\sum_{k \geq n^{2 / 3} /\left|\lambda_{n}\right|} P_{n, p}(k)=\sum_{k \geq n^{2 / 3} /\left|\lambda_{n}\right|} \frac{k}{k} P_{n, p}(k)=n^{-1 / 3} \times \begin{cases}O\left(e^{-\Theta\left(\left|\lambda_{n}\right|\right)}\right) & \text { if } \quad \lambda_{n}<0 \\ O\left(e^{-\Theta\left(\lambda_{n}^{-3 / 5}\right)}\right) & \text { if } \quad \lambda_{n}>0 .\end{cases}
$$

Proof of Lemma 6.5. To prove the lemma, we again consider three regions of $k: k \leq$ $n^{2 / 3} /\left|\lambda_{n}\right|, n^{2 / 3} /\left|\lambda_{n}\right| \leq k \leq n^{2 / 3}\left|\lambda_{n}\right|$, and $k \geq n^{2 / 3}\left|\lambda_{n}\right|$. In the first region, we use (5.23) to approximate $P_{n, p}(k)$ by $Q_{n, p}(k)$. Combined with Lemma 6.3, this gives

$$
\sum_{k \leq \frac{n^{2} / 3}{|\lambda|}} k^{a} P_{n, p}(k)=\frac{1+o_{\varepsilon, \lambda_{n}}(1)}{2 p n \sqrt{2 \pi}}\left[\frac{2 n^{2 / 3}}{\lambda_{n}^{2}}\right]^{a-1 / 2} \Gamma(a-1 / 2) .
$$

For $\lambda_{n}<0$, we combine the second and the third region. Using the fact that $P_{n, p}(k) \leq$ $Q_{n, p}(k)$ by (5.16), we then use Lemma 6.3 to obtains the bound (6.14) below threshold.

Above threshold, the contribution from the second region is

$$
\begin{array}{rlrl}
\sum_{n^{2 / 3} /\left|\lambda_{n}\right| \leq k \leq n^{2 / 3}\left|\lambda_{n}\right|} k^{a} P_{n, p}(k) & \leq \sum_{n^{2 / 3} /\left|\lambda_{n}\right| \leq k \leq n^{2 / 3}\left|\lambda_{n}\right|} k^{a} Q_{n, p}(k) & \text { by (5.16) } \\
& \leq\left(n^{2 / 3}\left|\lambda_{n}\right|\right)^{a} \sum_{n^{2 / 3} /\left|\lambda_{n}\right| \leq k \leq n^{2 / 3}\left|\lambda_{n}\right|} Q_{n, p}(k) \\
& \leq\left(n^{2 / 3}\left|\lambda_{n}\right|\right)^{a} \exp \left[-\Theta\left(\left|\lambda_{n}\right|\right)\right] n^{-1 / 3} & \text { by }(5.25) \\
& =\left[\frac{2 n^{2 / 3}}{\lambda_{n}^{2}}\right]^{a-1 / 2} \times \exp \left[-\Theta\left(\left|\lambda_{n}\right|\right)\right] .
\end{array}
$$


In the third region we use (5.18) to write

$$
\sum_{k \geq n^{2 / 3}\left|\lambda_{n}\right|} k^{a} P_{n, p}(k)=O\left(\sum_{k \geq n^{2 / 3}\left|\lambda_{n}\right|} k^{a} \ell_{0}(k) 2^{-\ell_{0}(k)} Q_{n, p}(k)\right) .
$$

Consider the prefactor, i.e. the summand ignoring the factor of $Q_{n, p}(k)$. Provided $\ell_{0}(k+$ 1) $<n^{1 / 5}$, recalling the definition of $\ell_{0}$ in (5.19), we can differentiate the logarithm of the prefactor to get

$$
\frac{d}{d k} \log \left[k^{a+3} p^{2} /\left(12(1-p)^{2}\right) 2^{-k^{3} p^{2} /\left(12(1-p)^{2}\right)}\right]=\frac{a+3}{k}-(\log 2) 3 k^{2} \frac{p^{2}}{12(1-p)^{2}}
$$

which will be nonpositive provided

$$
\frac{4(a+3)}{\log 2} \leq k^{3} p^{2}=\frac{k^{3}}{4 n^{2}}(1+\varepsilon)^{2},
$$

which will hold if $\left|\lambda_{n}\right|$ is large enough, where "large enough" depends upon $a$. Provided that these conditions are met, the prefactor takes on its largest value in the first term, $k=n^{2 / 3}\left|\lambda_{n}\right|$. Eventually, if $k$ gets large enough, $\ell_{0}(k)$ may stop increasing, and take on the value $n^{1 / 5}$. If $a \geq 0$ then the prefactor will then increase up to $n^{a+1 / 5} 2^{-n^{1 / 5}}$. Thus the maximum value of the prefactor is

$$
\max \left\{\left(n^{2 / 3} \lambda_{n}\right)^{a} \frac{\lambda_{n}{ }^{3}}{\left(48+o_{\varepsilon, \lambda_{n}}(1)\right)} 2^{-\lambda_{n}{ }^{3} /\left(48+o_{\varepsilon, \lambda_{n}}(1)\right)}, \quad n^{a+1 / 5} 2^{-n^{1 / 5}}\right\} .
$$

Since by assumption $\varepsilon=\lambda_{n} n^{-1 / 3} \leq 1$, we have $n \geq \Theta\left(\lambda_{n}{ }^{3}\right)$, so the second term in the $\max$ is $n^{a} \exp \left[-\Theta\left(n^{1 / 5}\right)\right] \exp \left[-\Theta\left(\lambda_{n}^{3 / 5}\right)\right]$. Thus we can write the maximum prefactor as

$$
\max \left\{n^{(2 / 3) a} \exp \left[-\Theta\left(\lambda_{n}^{3}\right)\right], \quad n^{a} \exp \left[-\Theta\left(n^{1 / 5}\right)\right] \exp \left[-\Theta\left(\lambda_{n}^{3 / 5}\right)\right]\right\} \leq n^{(2 / 3) a} \exp \left[-\Theta\left(\lambda_{n}^{3 / 5}\right)\right] .
$$

Upon substituting the above expression into (6.19), we find

$$
\begin{aligned}
\sum_{k \geq n^{2 / 3}\left|\lambda_{n}\right|} k^{a} P_{n, p}(k) & \leq \sum_{k \geq n^{2 / 3}\left|\lambda_{n}\right|} n^{(2 / 3) a} \exp \left[-\Theta\left(\lambda_{n}^{3 / 5}\right)\right] Q_{n, p}(k) \\
& \leq n^{(2 / 3) a-1 / 3} \exp \left[-\Theta\left(\lambda_{n}^{3 / 5}\right)\right] \\
& =\left[\frac{2 n^{2 / 3}}{\lambda_{n}^{2}}\right]^{a-1 / 2} \exp \left[-\Theta\left(\lambda_{n}^{3 / 5}\right)\right]
\end{aligned}
$$

where we used (5.24) to get the second line.

The bounds (6.18) and (6.20) imply (6.14) above threshold, and (6.14) and (6.17) imply the estimate $(6.13)$. 


\section{Expected Size of Spine}

After the preparation of the last three sections, we are ready to prove the bounds (3.4) and (3.5) in Theorems 3.1.

Proof of Theorem 3.1. We begin with a derivation which holds for $\varepsilon$ of both signs, and later distinguish $\varepsilon<0$ from $\varepsilon>0$. Since $\sum_{k \geq 1} Q_{n, p}(k)=1$, we have

$$
\mathbb{P}\left(x \underset{F_{n, p}}{\rightsquigarrow} \bar{x}\right)=1-\sum_{k \geq 1} P_{n, p}(k)=\sum_{k}\left(Q_{n, p}(k)-P_{n, p}(k)\right) .
$$

By (5.17),

$$
\begin{aligned}
\sum_{k \leq \frac{n^{2} / 3}{|\lambda|}}\left(Q_{n, p}(k)-P_{n, p}(k)\right) & =\sqrt{\frac{\pi}{8}} \sum_{k \leq n^{2 / 3}} \frac{p}{1-p} k^{3 / 2}\left[1+O\left(k^{-1 / 2}\right)+O\left(\frac{k^{3 / 2} p}{1-p}\right)\right] P_{n, p}(k) \\
& =\sqrt{\frac{\pi}{8}}\left(1+o_{\lambda_{n}}(1)\right) \sum_{k \leq \frac{n^{2} / 3}{|\lambda|}} p\left[k^{3 / 2}+O(k)\right] P_{n, p}(k) \\
& =\sqrt{\frac{\pi}{8}}\left(1+o_{\lambda_{n}}(1)\right)\left[\left(1+o_{\varepsilon, \lambda_{n}}(1)\right) \frac{p}{2 p n} \frac{\sqrt{2 / \pi}}{\varepsilon^{2}}+O(p / \varepsilon)\right] \\
& =\frac{1+o_{\varepsilon, \lambda_{n}}(1)}{4 n \varepsilon^{2}}=\frac{1+o_{\varepsilon, \lambda_{n}}(1)}{4 n^{1 / 3} \lambda_{n}{ }^{2}}
\end{aligned}
$$

So far everything we have done in this section holds for both the subcritical region and the supercritical region. We now consider these regions separately.

Subcritical regime:

We use (6.7) to sum over the remaining values of $k$ :

$$
0 \leq \sum_{k>n^{2 / 3} /\left|\lambda_{n}\right|}\left(Q_{n, p}(k)-P_{n, p}(k)\right) \leq \sum_{k>n^{2 / 3} /\left|\lambda_{n}\right|} Q_{n, p}(k)=O\left(e^{-c\left|\lambda_{n}\right|} n^{-1 / 3}\right) .
$$

Putting these two ranges together we get

$$
\begin{aligned}
\mathbb{P}\left(x \underset{F_{n, p}}{\rightsquigarrow} \bar{x}\right) & =\sum_{k}\left(Q_{n, p}(k)-P_{n, p}(k)\right) \\
& =\sum_{k \leq n^{2 / 3} /\left|\lambda_{n}\right|}\left(Q_{n, p}(k)-P_{n, p}(k)\right)+\sum_{n^{2 / 3} /\left|\lambda_{n}\right|<k}\left(Q_{n, p}(k)-P_{n, p}(k)\right) \\
& =\frac{1+o_{\varepsilon, \lambda_{n}}(1)}{4 n^{1 / 3} \lambda_{n}{ }^{2}}+O\left(\frac{e^{-c\left|\lambda_{n}\right|}}{n^{1 / 3}}\right) \\
& =\frac{1+o_{\varepsilon, \lambda_{n}}(1)}{4 n^{1 / 3} \lambda_{n}{ }^{2}},
\end{aligned}
$$


which completes the proof of (3.4).

Supercritical regime:

If $k$ is "mid-size," we can apply (5.16) and (5.25):

$$
0 \leq \sum_{n^{2 / 3} / \lambda_{n}<k \leq \lambda_{n} n^{2 / 3}}\left(Q_{n, p}(k)-P_{n, p}(k)\right) \leq \sum_{n^{2 / 3} / \lambda_{n}<k \leq \lambda_{n} n^{2 / 3}} Q_{n, p}(k)=O\left(e^{-c \lambda_{n}} / n^{1 / 3}\right) .
$$

If $k$ is "large," we proceed as in the proof of (6.20) to write

$$
\begin{aligned}
\sum_{k>\lambda_{n} n^{2 / 3}}\left(Q_{n, p}(k)-P_{n, p}(k)\right) & =\left(1-O\left(\exp \left[-\Theta\left(\lambda_{n}^{3 / 5}\right)\right]\right)\right) \sum_{k>\lambda_{n} n^{2 / 3}} Q_{n, p}(k) \\
& =\vartheta(\varepsilon)\left(1+o_{\lambda_{n}}(1)\right) \\
& =2 \lambda_{n} n^{-1 / 3}\left(1+o_{\lambda_{n}}(1)+O(\varepsilon)\right) .
\end{aligned}
$$

Putting these three ranges of $k$ together, we find

$$
\begin{aligned}
\mathbb{P}\left(x \underset{F_{n, p}}{x} \bar{x}\right) & =\sum_{k}\left(Q_{n, p}(k)-P_{n, p}(k)\right) \\
& =\left[\sum_{k \leq n^{2 / 3} / \lambda_{n}}+\sum_{n^{2 / 3} / \lambda_{n}<k \leq \lambda_{n} n^{2 / 3}}+\sum_{k>\lambda_{n} n^{2 / 3}}\right]\left(Q_{n, p}(k)-P_{n, p}(k)\right) \\
& =\frac{1+o_{\varepsilon, \lambda_{n}}(1)}{4 n^{1 / 3} \lambda_{n}{ }^{2}}+O\left(\frac{e^{-c \lambda_{n}}}{n^{1 / 3}}\right)+\vartheta(\varepsilon)\left(1+o_{\lambda_{n}}(1)\right) \\
& =\vartheta(\varepsilon)\left(1+o_{\lambda_{n}}(1)\right) \\
& =2 \lambda_{n} n^{-1 / 3}\left(1+o_{\lambda_{n}}(1)+O(\varepsilon)\right)
\end{aligned}
$$

which yields (3.5) and completes the proof of Theorem 3.1.

\section{Variance of the Size of the Spine}

In this section we shall prove the bounds (3.6) and (3.7) on the variance of the size of the spine given in Theorem 3.2. First, we note that the lower bound is immediate from the Harris-Kleitman correlation inequality (see [Har60, [Kle66]), which was later generalized to the FKG inequality FKG71. We use $\underset{n}{x} \bar{x}$ as a shorthand for $\underset{F_{n, p}}{\rightsquigarrow} \bar{x}$, and if $M$ is a set of literals, $\underset{M}{\rightsquigarrow} \bar{x}$ means that there is a path from $x$ to $\bar{x}$ using only literals in $M$.

Lemma 8.1. For strictly distinct literals $x, y$, we have

$$
\mathbb{P}(\underset{n}{x} \bar{x}, y \underset{n}{\rightsquigarrow} \bar{y})-\mathbb{P}(\underset{n}{\rightsquigarrow} \bar{x})^{2} \leq \sum_{k \geq 1} P_{n, p}(k)\left(\underset{n}{\mathbb{P}}(\underset{n}{x})-\frac{n-k}{n-1} \mathbb{P}\left(x_{n-k}^{\rightsquigarrow} \bar{x}\right)\right) .
$$


Proof. Let $\widehat{P}_{n, p}(k ; y)$ denote the event that $L^{+}(y)$ is strictly distinct, and $\left|L^{+}(y)\right|=k$. So in particular $\mathbb{P}\left(\widehat{P}_{n, p}(k ; y)\right)=P_{n, p}(k)$. Using the resolution of the identity

$$
1=\mathbb{I}_{y \rightsquigarrow \bar{y}}+\sum_{k=1}^{\infty} \mathbb{I}_{\widehat{P}_{n, p}(k ; y)},
$$

where $\mathbb{I}_{A}$ denotes the indicator function of the event $A$, we can decompose $\mathbb{P}(x \rightsquigarrow \bar{x})$ in two different ways to obtain

$$
\mathbb{P}(\underset{n}{x} \bar{x}, \underset{n}{\rightsquigarrow} \bar{y})+\sum_{k} \mathbb{P}\left(\underset{n}{\rightsquigarrow} \bar{x}, \widehat{P}_{n, p}(k ; y)\right)=\mathbb{P}(\underset{n}{x \rightsquigarrow} \bar{x})\left(\mathbb{P}(\underset{n}{\rightsquigarrow} \bar{y})+\sum_{k} \mathbb{P}\left(\widehat{P}_{n, p}(k ; y)\right)\right),
$$

so that

$$
\mathbb{P}(\underset{n}{x} \bar{x}, \underset{n}{\rightsquigarrow} \bar{y})-\mathbb{P}(\underset{n}{\rightsquigarrow} \bar{x}) \mathbb{P}(\underset{n}{\underset{n}{\rightsquigarrow}} \bar{y})=\sum_{k}\left(\mathbb{P}(\underset{n}{x} \bar{x})-\mathbb{P}\left(\underset{n}{x} \bar{x} \mid \widehat{P}_{n, p}(k ; y)\right)\right) P_{n, p}(k) .
$$

To estimate the probability on the right, consider the event $\widehat{P}_{n, p}(k ; y)$. With probability $(k-1) /(n-1)$ either $x$ or $\bar{x}$ is in $L^{+}(y)$. If $x \in L^{+}(y)$ then $x \not y \rightarrow \bar{x}$. If $\bar{x} \in L^{+}(y)$, the situation is more complicated, so we shall bound the probability below by 0 . If $L^{+}(y)$ contains neither $x$ nor $\bar{x}$, then any path from $x$ to $\bar{x}$ avoids literals in $L^{+}(y)$. In this case we may as well explore the out-graph $L^{+}(x)$ restricted to avoid the variables in $L^{+}(y)$; with probability $\mathbb{P}\left(x_{n-k}^{\rightsquigarrow} \bar{x}\right)$ the restricted out-graph will contain $\bar{x}$. Thus we conclude

$$
\begin{aligned}
\mathbb{P}\left(x \underset{n}{\rightsquigarrow} \mid \widehat{P}_{n, p}(k ; y)\right) & =\frac{n-k}{n-1} \mathbb{P}(\underbrace{\rightsquigarrow}_{n-k} \bar{x})+\frac{1}{2} \frac{k-1}{n-1} \mathbb{P}\left(\underset{n}{\rightsquigarrow} \bar{x} \mid \bar{x} \in L^{+}(y), \widehat{P}_{n, p}(k ; y)\right) \\
& \geq \frac{n-k}{n-1} \mathbb{P}(x \underset{n-k}{\rightsquigarrow}) .
\end{aligned}
$$

Substituting this into (8.1) proves the lemma.

Next we relate the probabilities of the events $\underset{n-k}{x} \bar{x}$ and $\underset{n}{x} \bar{x}$.

Lemma 8.2. If $\lambda_{n}=\varepsilon n^{1 / 3}$ is large enough, and $\varepsilon=\lambda_{n} n^{-1 / 3}$ is small enough, then

$$
\mathbb{P}(x \underset{n+1}{\rightsquigarrow} \bar{x})-\mathbb{P}(\underset{n}{x} \bar{x}) \leq \begin{cases}\frac{1+o_{\varepsilon, \lambda_{n}}(1)}{2 n\left|\lambda_{n}\right|^{3}} & \text { if } \lambda_{n}<0 \\ \frac{2+o_{\varepsilon, \lambda_{n}}(1)}{n} & \text { if } \lambda_{n}>0 .\end{cases}
$$

Proof. Suppose $x \underset{n}{\not \rightarrow} \bar{x}$. Let $X$ denote $L_{n, p}^{+}(x)$, which then must be strictly distinct. We shall consider four cases depending on whether or not $X \rightarrow x_{n+1}$ and whether or not $X \rightarrow \bar{x}_{n+1}$ : 
Case 1. $X \not \rightarrow x_{n+1}$ and $X \not \rightarrow \bar{x}_{n+1}$. Then $x \not h \rightarrow \bar{x}$.

Case 2. $X \rightarrow x_{n+1}$ and $X \rightarrow \bar{x}_{n+1}$. Then $x \underset{n+1}{n+1} \bar{x}$.

Case 3. $X \rightarrow x_{n+1}$ and $X \not \rightarrow \bar{x}_{n+1}$. It is clear that if $x_{n+1} \underset{[n+1] \backslash[X]}{\rightsquigarrow} \bar{x}_{n+1}$, where $[X]$ denotes $X \cup \bar{X}$, then $x \underset{n+1}{\rightsquigarrow} \bar{x}$. Suppose that conversely $\underset{n+1}{x} \bar{x}$. Since $x \underset{n}{\not} \bar{x}$, either $x_{n+1}$ or $\bar{x}_{n+1}$ is in the path from $x$ to $\bar{x}$ in $[n+1]$. The first such occurence must be $x_{n+1}$, so we have $x_{n+1} \underset{n+1}{\rightsquigarrow} \bar{x}$, and its contrapositive $x \underset{n+1}{\rightsquigarrow} \bar{x}_{n+1}$. Since $x \not 4 \bar{x}_{n+1}$ within $[n] \cup\left\{\bar{x}_{n+1}\right\}$, it must be that $x_{n+1}$ occurs in the path from $x$ to $\bar{x}_{n+1}$. In particular $x_{n+1} \underset{n+1}{\rightsquigarrow} \bar{x}_{n+1}$. We may assume without loss of generality that $x_{n+1}$ and $\bar{x}_{n+1}$ occur only at the endpoints of this path. If a literal in $X$ occurred in the path from $x_{n+1}$ to $\bar{x}_{n+1}$, consider the last such one. The next literal cannot be $\bar{x}_{n+1}$ by assumption, nor can it be $x_{n+1}$, since this occurs only at the beginning of the path. So the next literal would have to lie inside $X$, a contradiction. If a literal in $\bar{X}$ occured in the path from $x_{n+1}$ to $\bar{x}_{n+1}$, we could take the contrapositive and similarly derive a contradiction. Thus $x_{n+1} \rightsquigarrow \bar{x}_{n+1}$ using only literals in $[n+1] \backslash[X]$, i.e. $x_{n+1} \underset{[n+1] \backslash[X]}{\rightsquigarrow} \bar{x}_{n+1}$. Thus conditional upon $L_{n}^{+}(x)=X$, we can write

$$
\begin{aligned}
& X \rightarrow x_{n+1}, X \not \rightarrow \bar{x}_{n+1}, x \underset{n+1}{\rightsquigarrow} \bar{x} \text { iff } X \rightarrow x_{n+1}, X \not \rightarrow \bar{x}_{n+1}, x_{n+1} \underset{[n+1] \backslash[X]}{\rightsquigarrow} \bar{x}_{n+1} \\
& \mathbb{P}\left[X \rightarrow x_{n+1}, X \not \rightarrow \bar{x}_{n+1}, x \underset{n+1}{\rightsquigarrow} \bar{x} \mid L_{n}^{+}(x)=X\right]= \\
& \mathbb{P}\left[X \rightarrow x_{n+1}\right] \mathbb{P}\left[X \not \rightarrow \bar{x}_{n+1}\right] \mathbb{P}\left[x_{n+1} \underset{[n+1] \backslash[X]}{\rightsquigarrow} \bar{x}_{n+1}\right]
\end{aligned}
$$

since the events on the right and the event $L_{n}^{+}(x)=X$ are determined by pairwise disjoint sets of variables.

Case 4. $X \nrightarrow \rightarrow x_{n+1}$ and $X \rightarrow \bar{x}_{n+1}$. By symmetry, cases 3 and 4 have the same probability.

Putting these four cases together we see

$$
\begin{aligned}
\mathbb{P}\left(x \underset{n+1}{\rightsquigarrow} \bar{x} \mid L_{n, p}^{+}(x)=X\right) & =\left[1-(1-p)^{|X|}\right]^{2}+2\left[1-(1-p)^{|X|}\right](1-p)^{|X|} \mathbb{P}\left[x_{n+1-|X|}^{\rightsquigarrow} \bar{x}\right] \\
& \leq p^{2}|X|^{2}+2 p|X| \mathbb{P}\left[x_{n+1-|X|}^{\rightsquigarrow} \bar{x}\right] .
\end{aligned}
$$

As a consequence,

$$
\begin{aligned}
\mathbb{P}(x \underset{n+1}{x} \bar{x})-\mathbb{P}(\underset{n}{x} \bar{x})= & \mathbb{P}(\underset{n+1}{\rightsquigarrow} \bar{x} \backslash x \underset{n}{n}) \\
= & \sum_{\substack{X \subset[n] \\
X \text { strictly distinct }}} \mathbb{P}\left(L_{n, p}^{+}(x)=X\right) \mathbb{P}\left(x \underset{n+1}{\rightsquigarrow} \bar{x} \mid L_{n, p}^{+}(x)=X\right) \\
\leq & \sum_{k} P_{n, p}(k)\left(p^{2} k^{2}+2 p k \mathbb{P}[\underset{n+1-k}{x} \bar{x}]\right) \\
\leq & p^{2} \sum_{k} P_{n, p}(k) k^{2}+2 p \mathbb{P}\left[\underset{n}{x \underset{x}{x}] \sum_{k}} P_{n, p}(k) k .\right.
\end{aligned}
$$


Hence by (6.15), we have

$$
\begin{aligned}
\mathbb{P}(x \underset{n+1}{\rightsquigarrow} \bar{x})-\mathbb{P}(x \underset{n}{\rightsquigarrow}) & \leq p^{2} \frac{1+o(1)}{2 p n} \frac{1}{|\varepsilon|^{3}}+2 p \mathbb{P}[\underset{n}{x} \bar{x}] \frac{1+o(1)}{2 p n} \frac{1}{|\varepsilon|} \\
& =\frac{1+o(1)}{4 n^{2}|\varepsilon|^{3}}+\frac{1+o(1)}{n|\varepsilon|} \times \begin{cases}\frac{1+o(1)}{4 n \varepsilon^{2}} & \text { if } \lambda_{n}<0 \text { by (3.4) } \\
(2+o(1)) \varepsilon & \text { if } \lambda_{n}>0 \text { by (3.5) }\end{cases} \\
& =[1+o(1)] \times \begin{cases}1 /\left[2 n^{2}|\varepsilon|^{3}\right] & \text { if } \lambda_{n}<0, \\
2 / n & \text { if } \lambda_{n}>0 .\end{cases} \\
& =[1+o(1)] \times \begin{cases}1 /\left[2 n\left|\lambda_{n}\right|^{3}\right] & \text { if } \lambda_{n}<0, \\
2 / n & \text { if } \lambda_{n}>0 .\end{cases}
\end{aligned}
$$

In the above, all of the $o(1)$ terms are $o_{\varepsilon, \lambda_{n}}(1)$.

Corollary 8.3. Provided $k \leq n^{2 / 3} /\left|\lambda_{n}\right|$, we have

$$
\operatorname{Pr}(\underset{n}{\rightsquigarrow} \bar{x})-\mathbb{P}(x \underset{n-k}{\rightsquigarrow} \bar{x}) \leq \begin{cases}\left(1+o_{\varepsilon, \lambda_{n}}(1)\right) \frac{k}{2 n\left|\lambda_{n}\right|^{3}} & \text { if } \lambda_{n}<0 \\ \left(1+o_{\varepsilon, \lambda_{n}}(1)\right) \frac{2 k}{n} & \text { if } \lambda_{n}>0 .\end{cases}
$$

Proof. We seek

$$
\mathbb{P}(x \underset{n}{x} \bar{x})-\mathbb{P}(x \underset{n-k}{\rightsquigarrow} \bar{x})=\sum_{m=n-k}^{n-1}[\mathbb{P}(x \underset{m+1}{\rightsquigarrow} \bar{x})-\mathbb{P}(\underset{m}{x} \bar{x})],
$$

and need only show that each summand is well-approximated by $\mathbb{P}(\underset{n+1}{\rightsquigarrow} \bar{x})-\mathbb{P}(\underset{n}{\rightsquigarrow} \bar{x})$. Define $\lambda_{n}{ }^{\prime}$ by

$$
p \equiv \frac{1+\lambda_{n} n^{-1 / 3}}{2 n}=\frac{1+\lambda_{n}{ }^{\prime} m^{-1 / 3}}{2 m}
$$

so that

$$
\frac{m}{n}\left[1+\lambda_{n} n^{-1 / 3}\right]-1=\lambda_{n}{ }^{\prime} m^{-1 / 3}
$$

and thus

$$
\frac{m-n}{n} m^{1 / 3}+\lambda_{n}\left(\frac{m}{n}\right)^{4 / 3}=\lambda_{n}{ }^{\prime} .
$$

Since $m=\left(1+o_{\varepsilon, \lambda_{n}}(1)\right) n$, and $|n-m| \leq k \ll n^{2 / 3}$, it follows that $\lambda_{n}{ }^{\prime}=\left(1+o_{\varepsilon, \lambda_{n}}(1)\right) \lambda_{n}$. Thus when we apply Lemma 8.2 with $m$ and $\lambda_{n}{ }^{\prime}$ rather than $n$ and $\lambda_{n}$, we obtain an answer that differs by a factor of $1+o_{\varepsilon, \lambda_{n}}(1)$, as desired. 
Proof of Theorem 3.9. We can now complete our estimate of the covariances. For strictly distinct literals $x$ and $y$ we have

$$
\begin{aligned}
& \operatorname{Cov}(\underset{n}{\rightsquigarrow} \bar{x}, y \underset{n}{\rightsquigarrow} \bar{y})=\mathbb{P}(\underset{n}{x} \bar{x}, \underset{n}{\rightsquigarrow} \bar{y})-\mathbb{P}(\underset{n}{x \rightsquigarrow} \bar{x}) \mathbb{P}(y \underset{n}{\rightsquigarrow} \bar{y}) \\
& \leq \sum_{k \geq 1} P_{n, p}(k)\left(\mathbb{P}(\underset{n}{x} \bar{x})-\frac{n-k}{n-1} \mathbb{P}(\underset{n-k}{\rightsquigarrow} \bar{x})\right) \quad \text { by Lemma } 8.1 \\
& =\sum_{k \geq 1} P_{n, p}(k) \frac{k-1}{n-1} \mathbb{P}(\underset{n}{x} \bar{x})+\sum_{k \geq 1} P_{n, p}(k) \frac{n-k}{n-1}\left(\mathbb{P}(\underset{n}{x \rightsquigarrow} \bar{x})-\mathbb{P}\left(x_{n-k}^{\rightsquigarrow} \bar{x}\right)\right) \\
& \leq \mathbb{P}(\underset{n}{x} \bar{x}) \frac{1}{n} \sum_{k \geq 1} P_{n, p}(k) k+\sum_{k \geq 1} P_{n, p}(k)\left(\mathbb{P}(\underset{n}{x \rightsquigarrow \bar{x}})-\mathbb{P}\left(x_{n-k}^{\rightsquigarrow} \bar{x}\right)\right) .
\end{aligned}
$$

As a consequence,

$$
\begin{aligned}
& \operatorname{Cov}(\underset{n}{x} \bar{x}, y \underset{n}{\rightsquigarrow} \bar{y}) \leq \\
& \leq \mathbb{P}(\underset{n}{x \rightsquigarrow} \bar{x}) \frac{1}{n} \sum_{k \geq 1} P_{n, p}(k) k+\sum_{k \geq 1} P_{n, p}(k)\left(\mathbb{P}(\underset{n}{x} \bar{x})-\mathbb{P}\left(x_{n-k}^{\rightsquigarrow} \bar{x}\right)\right) \\
& =\mathbb{P}(\underset{n}{x \rightsquigarrow \bar{x}}) \frac{1+o(1)}{n \varepsilon}+\left[\sum_{k \leq n^{2 / 3} /\left|\lambda_{n}\right|}+\sum_{k>n^{2 / 3} /\left|\lambda_{n}\right|}\right] P_{n, p}(k)(\mathbb{P}(\underset{n}{x \rightsquigarrow \bar{x}})-\mathbb{P}(x \underset{n-k}{\rightsquigarrow} \bar{x})) \\
& \leq \mathbb{P}(\underset{n}{\rightsquigarrow} \bar{x}) \frac{1+o(1)}{n \varepsilon}+\sum_{k \leq n^{2 / 3} /\left|\lambda_{n}\right|} P_{n, p}(k)\left\{\begin{array}{c}
\frac{(1+o(1)) k}{2 n\left|\lambda_{n}\right|^{3}} \\
\frac{(2+o(1)) k}{n}
\end{array}\right\}+\sum_{k>n^{2 / 3} /\left|\lambda_{n}\right|} P_{n, p}(k) \mathbb{P}\left(x_{n} \bar{x}\right) \\
& =\left\{\begin{array}{c}
\frac{1+o(1)}{4 \varepsilon^{2} n} \\
(2+o(1)) \varepsilon
\end{array}\right\} \frac{1+o(1)}{n \varepsilon}+\left\{\begin{array}{c}
\frac{1+o(1)}{2 n\left|\lambda_{n}\right|^{3} \varepsilon} \\
\frac{2+o(1)}{n \varepsilon}
\end{array}\right\}+\left\{\begin{array}{c}
\exp \left[-\Theta\left(\lambda_{n}\right)\right] n^{-1 / 3} \times \frac{1+o(1)}{4 \varepsilon^{2} n} \\
\exp \left[-\Theta\left(\lambda_{n}{ }^{3 / 5}\right)\right] n^{-1 / 3} \times(2+o(1)) \varepsilon
\end{array}\right\} \\
& =\left\{\begin{array}{l}
\frac{1+o(1)}{2 n\left|\lambda_{n}\right|^{3} \varepsilon} \\
\frac{2+o(1)}{n \varepsilon}
\end{array}\right\}=\left\{\begin{array}{l}
\frac{1+o(1)}{2 n^{2 / 3} \lambda_{n}^{4}} \\
\frac{2+o(1)}{n^{2 / 3} \lambda_{n}}
\end{array}\right\}
\end{aligned}
$$

where the upper entry applies to the subcritical regime, and the lower entry applies to the supercritical regime. In the above, the $o(1)$ terms are all $o_{\varepsilon, \lambda_{n}}(1)$. This gives the variance bound above threshold. The bound on the second moment below threshold follows by combining the above variance bound with Theorem 3.1. 


\section{The Existence of Hourglasses}

In this section we prove Theorem 4.3, which was central to the proof of the upper bounds in Theorem 3.4.

Proof of Theorem 4.3 (i) on the existence of many hourglasses in the subcritical regime.

Let $t$ be a large positive number, but still small compared to $n^{1 / 3}$, and let $p=(1-$ $\left.t n^{-1 / 3}\right) / 2 n$. We shall construct a process for growing hourglasses one by one. However, as we deplete variables, the distribution changes, so that the hourglasses so constructed are not identically distributed. We therefore use the following two variations of this naive procedure: In the first process, instead of drawing from $n$ variables, we draw only from $n^{\prime}=n-t n^{2 / 3}$ variables, so that we have a "buffer" of $t n^{2 / 3}$, and replenish the variables as necessary. However, even this process can lead to trouble in the unlikely event that we have a very long run which uses up our entire reserve of variables. So we construct another process which aborts the growth of an hourglass when it would use up too many variables.

To be explicit, consider the trimmed out-graphs and trimmed in-graphs of various literals, where the in- and out-graphs are restricted to sets of $n^{\prime}=n-t n^{2 / 3}$ variables. Recall from Lemma 2.6 that the unoriented projection of the trimmed out-graph $\widetilde{D}_{F_{n^{\prime}, p}}^{+}(x)$ of a literal $x$ is identically distributed to the connected component $C_{n^{\prime}, 2 p-p^{2}}(x)$ in $G_{n^{\prime}, 2 p-p^{2}}$. We shall follow the convention that no matter how many trimmed out-graphs or trimmed in-graphs we have explored in the past, when exploring another in-graph or out-graph, we shall always restrict our attention to variables that are in none of the in-graphs or outgraphs found so far (except, possibly, for the root), and we shall add variables to ensure that there are $n^{\prime}=n-t n^{2 / 3}$ variables for the tree to grow within. (Recall that for the upper bounds we assume that we have a variable for each natural number.) In this way the sizes and structures of all the trees will be independent and identically distributed. As we alluded to above, later we shall consider a variation on this process.

Since there are somewhat fewer than $n$ variables in which we explore the out-graphs and in-graphs, this decreases the average out-degree of each literal, and has the effect of shifting the formula further into the subcritical regime. Specifically, if we define $t^{\prime}$ by

$$
\frac{1-t n^{-1 / 3}}{2 n}=p=\frac{1-t^{\prime}\left(n^{\prime}\right)^{-1 / 3}}{2 n^{\prime}}
$$

we see that $t^{\prime}=(2+o(1)) t$.

Pick a literal $u$, and look at its trimmed out-graph $T$ within $n^{\prime}$ unused variables. Recall the definition of $R_{n, p}(k)$, the probability that $C_{n, 2 p-p^{2}}(u)$ is a tree of size $k$. By using Lemma 6.1 for $R_{n, p}(k)$, we see that, for some $c$, there is a $(c+o(1))\left(t / n^{1 / 3}\right)$ chance that $T$ is a tree of size between $2 n^{2 / 3} / t^{2}$ and $4 n^{2 / 3} / t^{2}$. Here, as explained in the first sentence of the proof, we are assuming $1 \ll t \ll n^{1 / 3}$, so by Remark 6.2, in the remainder of this proof all $o(1)$ terms without subscripts are of the form $o_{t / n^{1 / 3}, t}(1)$. By comparing with random graphs, if $T$ is a tree, then it is uniformly distributed amongst the spanning trees on $|T|$ vertices. Using the structural properties of random spanning trees (see e.g. [AId90]), if 
we pick a random vertex $w \neq u$ in $T$, with probability at least $\left(1-o_{|T|}(1)\right) e^{-1}$ the path connecting $u$ to $w$ has length at least $\sqrt{2|T|}$. Let $v$ be the middle vertex in the path from $u$ to $w$ (in case of tie, we choose the vertex closer to $w$ ). Either the majority of the remaining vertices are connected to the first part of the path or to the second part of the path. Since the spanning tree is uniformly random, by symmetry, with probability at least $1 / 2$, at least half of the remaining vertices of the tree will be connected to $v$ via the path from $v$ to $w$ - these vertices will be in the out-graph of $v$. In the event that the path from $u$ to $v$ has length at least $(|T| / 2)^{1 / 2} \geq n^{1 / 3} / t$, and $v$ has at least $\frac{1}{2}|T| \geq n^{2 / 3} / t^{2}$ descendents in the trimmed out-graph of $u$, say that vertex $v$ is "promising," and that the path from $u$ to $v$ is the tail. We thus have shown

$$
\mathbb{P}\left[\text { a random vertex } v \text { in } \widetilde{D}_{F_{n^{\prime}, p}}^{+}(u) \text { is promising }\right]=(1+o(1)) \frac{c}{2 e} \frac{t}{n^{1 / 3}} .
$$

In the event that vertex $v$ is promising, we proceed to explore the trimmed in-graphs of the first $n^{1 / 3} / t$ vertices on $v$ 's tail. Again by Lemma 6.1, each individual in-graph will have size at least $n^{2 / 3} / t^{2}$ with probability at least $(c+o(1))\left(t / n^{1 / 3}\right)$. The probability that none of them is so large is at most $(1+o(1)) e^{-c}$. If any of them is so large, we shall call $v$ a "central variable," and $v$ together with its explored out-graph and in-graph both of size at least $n^{2 / 3} / t^{2}$ an "hourglass" (see Definition 4.2). Each time that we pick a literal and look for an hourglass as described above, we find one with probability at least

$$
(1+o(1)) \frac{c\left(1-e^{-c}\right)}{2 e} \frac{t}{n^{1 / 3}} .
$$

Next let us compute how many variables we expect to use up while exploring the trimmed out-graph and trimmed in-graphs. At this point we recall from Lemma 6.3 and equation (6.6) that if $G$ is either the trimmed in-graph or trimmed out-graph of a vertex,

$$
E[|G|]=(1+o(1)) \frac{\left(n^{\prime}\right)^{1 / 3}}{t^{\prime}}=(1+o(1)) \frac{n^{1 / 3}}{2 t} .
$$

We always explore one out-graph, and with probability $(1+o(1))[c /(2 e)] t / n^{1 / 3}$ we explore $\left\lceil n^{1 / 3} / t\right\rceil$ in-graphs. Thus the expected number of variables used up is

$$
(1+o(1))\left[1+\frac{c}{2 e}\right] \frac{n^{1 / 3}}{2 t} .
$$

If we look for an hourglass for

$$
\frac{4 e}{c\left(1-e^{-c}\right)} n^{1 / 3} / t
$$

times, then the probability that we fail to find an hourglass is $(1+o(1)) e^{-2}$, and the expected number of variables that we use up is

$$
(1+o(1))\left[\frac{2 e}{c}+1\right] \frac{1}{1-e^{-c}} \frac{n^{2 / 3}}{t^{2}} .
$$


The probability that we use up more than $3(1+o(1))$ times the expected number of variables is at most $1 / 3+o(1)$. Therefore, with probability at least $1-e^{-2}-1 / 3-o(1) \geq$ $1 / 2$ (for large enough $t$ and small enough $t n^{-1 / 3}$ ) we both find an hourglass, and do not use up more than $b n^{2 / 3} / t^{2}$ variables, where $b=3[1+2 e / c] /\left(1-e^{-c}\right)$.

Now consider the following modification of the above procedure: as above we use the local search procedure in Section 2 to explore the trimmed out-graphs and trimmed ingraphs, hoping to find an hourglass, but as soon as we use up $b n^{2 / 3} / t^{2}$ variables, we abort and stop looking for the hourglass. Then we can repeat this procedure $t^{3} / b$ times, be guaranteed to use no variables other than the first $n$ of them, and find a number of disjoint hourglasses that stochastically dominates the binomial distribution $\operatorname{Binomial}\left(t^{3} / b, 1 / 2\right)$. By Chernoff's inequality [Che52] (see also McD89]), the probability that we find fewer than half as many hourglasses as we expect will be no larger than $\exp \left[-t^{3} /(8 b)\right]$.

Proof of Theorem 4.3 (ii) on the existence of a giant hourglass in the supercritical regime.

Let $t$ be a large positive number, but still small compared to $n^{1 / 3}$. When $p=(1-$ $\left.t n^{-1 / 3}\right) / 2 n$, as we have just seen, there will be $\Theta\left(t^{3}\right)$ hourglasses with in- and out-portion of size at least $n^{2 / 3} / t^{2}$, except with probability $\exp \left(-\Theta\left(t^{3}\right)\right)$. We now increase $p$ by a suitably large constant times $t n^{-4 / 3}$, say $M t n^{-4 / 3}$. For any two hourglasses, the probability of an edge from the out-portion of the first hourglass to the in-portion of the second hourglass is therefore at least $M / t^{3}$. Then the central variable of the first hourglass implies the central variable of the second hourglass. Conceptually we can think of the directed graph whose nodes are the hourglasses, and place a directed edge from one node to another whenever the hourglasses connect up like this. The edges of this graph occur independently of one another, and the average out-degree of the graph is $\Theta\left(M t^{3} / t^{3}\right)=\Theta(M)$. By choosing $M$ large enough, we can make the average out-degree to be any convenient constant that we like. In particular, if the average out-degree is a constant larger than 1 , then we might expect the connections to percolate, so that there is some node $v$ that can reach a constant fraction of the other nodes through edges of this graph, and is reachable by a constant fraction of the other nodes. Provided this happens, each literal in the out-portions of the nodes reachable by $v$ is implied by the central variable of node $v$, and each literal in the in-portions of the nodes that can reach $v$ will imply the central variable of $v$, thereby giving the desired giant hourglass with in-portion and out-portion each of size $\Theta\left(t n^{2 / 3}\right)$.

It remains to be shown (in Lemma 9.1) that we get the requisite percolation except with probability that is exponentially small in the number of nodes of the graph.

The following lemma is related to one proved by Karp in KKar90 which showed that with high probability there is a giant component of size $\Theta(N)$ in supercritical directed percolation. For our purposes "with high probability" is not sufficient; we need the exceptional events to be exponentially rare.

Lemma 9.1. In a random directed graph on $N$ vertices, in which each directed edge occurs independently with probability $6 / N$, then except with probability that is exponentially small in $\Theta(N)$, there is a vertex $v$ with out-graph of size $\Theta(N)$ and in-graph of size $\Theta(N)$. 
Proof. For convenience, let $N^{\prime}=\lceil N / 3\rceil$, so that there are at least $3 N^{\prime}-2$ vertices, and the probability of each directed edge is at least $2 / N^{\prime}$. (We can throw out some of the edges to make the probability exactly $2 / N^{\prime}$.) To search for a node $v$ with a large in-graph and out-graph, we consider candidate vertices one at a time, and explore the in-graph and out-graph of the candidate, restricting the explorations of the in-graph and out-graph to disjoint sets of $N^{\prime}-1$ vertices, none of which have yet been explored in the course of examining a previous failed candidate. In this way we ensure that the sizes of the in-graph and out-graph are independent, and both distributed in the same manner as the size of the component containing a particular vertex in the random graph $G_{N^{\prime}, 2 / N^{\prime}}$. We do the explorations in a parallel interleaved fashion, so that if either the in-graph or out-graph is found to be too small, then exploration of the other is immediately halted. We shall show that for large enough $N^{\prime}$, except with probability exponentially small in $N^{\prime}$, after looking at $N^{\prime} / 50$ candidates, the failed candidates have not wasted more than $N^{\prime}$ variables, and we find a successful candidate with in-graph and out-graph each of size at least $N^{\prime} / 10$.

We first claim that for any real $\gamma$ and integers $k$ and $N^{\prime}$ such that $0 \leq \gamma \leq N^{\prime}$ and $1 \leq k \leq N^{\prime}$, there is an $s$ between $1 / 2$ and 1 so that

$$
\mathbb{P}\left(|C(x)|=k \text { in } G_{N^{\prime}, \gamma / N^{\prime}}\right)=k^{k-2}\left(\begin{array}{c}
N^{\prime}-1 \\
k-1
\end{array}\right)\left(\frac{\gamma}{N^{\prime}}\right)^{k-1}\left(1-\frac{\gamma}{N^{\prime}}\right)^{k N^{\prime}-s k^{2}} .
$$

Indeed, the probability that the component $C(x)$ containing the vertex $x$ has size $k$ can be bounded below by the probability that $C(x)$ is a tree of size $k$. To bound $\mathbb{P}(|C(x)|=k)$ from above, we note that the connectedness of $C(x)$ implies that $C(x)$ contains a tree of size $k$. Summing over all possibilities for this spanning tree, we therefore get

$$
\sum_{\substack{\operatorname{trees} T \ni x \\|T|=k}} \mathbb{P}(T=C(x)) \leq \mathbb{P}(|C(x)|=k) \leq \sum_{\substack{\text { trees } T \ni x \\|T|=k}} \mathbb{P}(T \subseteq C(x),|T|=|C(x)|) .
$$

This give a lower bound of

$$
k^{k-2}\left(\begin{array}{c}
N^{\prime}-1 \\
k-1
\end{array}\right)\left(\frac{\gamma}{N^{\prime}}\right)^{k-1}\left(1-\frac{\gamma}{N^{\prime}}\right)^{k\left(N^{\prime}-k\right)+\left(\left(\begin{array}{c}
k \\
2
\end{array}\right)-(k-1)\right)}
$$

and an upper bound of

$$
k^{k-2}\left(\begin{array}{c}
N^{\prime}-1 \\
k-1
\end{array}\right)\left(\frac{\gamma}{N^{\prime}}\right)^{k-1}\left(1-\frac{\gamma}{N^{\prime}}\right)^{k\left(N^{\prime}-k\right)},
$$

which establishes the claim (9.1).

Next define $X$ by

$$
X= \begin{cases}|C(x)| & \text { if }|C(x)|<N^{\prime} / 10 \\ 0 & \text { if }|C(x)| \geq N^{\prime} / 10\end{cases}
$$


Using the bound (9.1) on $\mathbb{P}(|C(x)|=k)$ we see that

$$
\begin{aligned}
\mathbb{P}(|C(x)|=k) & \leq k^{k-2}\left(\begin{array}{c}
N^{\prime}-1 \\
k-1
\end{array}\right)\left(\frac{\gamma}{N^{\prime}}\right)^{k-1}\left(1-\frac{\gamma}{N^{\prime}}\right)^{k N^{\prime}-k^{2}} \\
& \leq \frac{k^{k-1}}{k !} \gamma^{k-1} e^{-k \gamma+\gamma k^{2} / N^{\prime}}
\end{aligned}
$$

For $k \leq N^{\prime} / 10$, we get

$$
\mathbb{P}(|C(x)|=k) \leq \frac{k^{k-1} e^{-k}}{k !} \frac{1}{\gamma} e^{k(1-\gamma+\log \gamma+\gamma / 10)} .
$$

Assuming $\gamma=2$, this gives

$$
\mathbb{P}(|C(x)|=k) \leq \frac{k^{k-1} e^{-k}}{k !} \frac{1}{2} e^{-k / 10}
$$

so that

$$
\sum_{k=1}^{N^{\prime} / 10} \mathbb{P}(|C(x)|=k) e^{k / 10} \leq \sum_{k=1}^{N^{\prime} / 10} \frac{k^{k-1} e^{-k}}{k !} \frac{1}{2} \leq \frac{1}{2} \sum_{k=1}^{\infty} \frac{k^{k-1} e^{-k}}{k !}=\frac{1}{2}
$$

Consequently

$$
E\left[e^{X / 10}\right] \leq \frac{1}{2}+\mathbb{P}\left(|C(x)| \geq N^{\prime} / 10\right) \times e^{0 / 10} \leq 3 / 2 .
$$

Let $Y_{i}$ be the number of variables lost on the $i$ th candidate if it is a failure; $Y_{i}=0$ if the $i$ th candidate is successful:

$$
Y_{i}= \begin{cases}0 & i \text { th candidate successful } \\ 2 k-1 & i \text { th candidate failed because in-graph had size } k<N^{\prime} / 10 \\ 2 k & i \text { th candidate failed because out-graph had size } k<N^{\prime} / 10\end{cases}
$$

Thus $\mathbb{P}(Y=j) \leq \mathbb{P}(X=\lceil j / 2\rceil)$, and hence

$$
E\left[e^{Y_{i} / 20}\right]=\sum_{j} \mathbb{P}\left(Y_{i}=j\right) e^{j / 20} \leq 2 \sum_{j \text { even }} \mathbb{P}(X=j / 2) e^{j / 20}=2 E\left[e^{X / 10}\right] \leq 3
$$

Letting

$$
S=\sum_{i=1}^{\beta N^{\prime}} Y_{i}
$$


be the total number of variables lost on failed candidates, we see

$$
\begin{aligned}
\mathbb{P}\left(S>N^{\prime}\right) & =\mathbb{P}\left(e^{S / 20}>e^{N^{\prime} / 20}\right) \\
& \leq \frac{E\left[e^{S / 20}\right]}{e^{N^{\prime} / 20}} \\
& =\frac{E\left[e^{Y / 20}\right]^{\beta N^{\prime}}}{e^{N^{\prime} / 20}} \\
& \leq \frac{3^{\beta N^{\prime}}}{e^{N^{\prime} / 20}}
\end{aligned}
$$

which is exponentially small in $N^{\prime}$ for $\beta=1 / 50$.

Next consider $\mathbb{P}\left(|C(x)| \geq N^{\prime} / 10\right)$. By (9.2) and (9.3) we have

$$
\begin{aligned}
\mathbb{P}\left(|C(x)| \geq \frac{N^{\prime}}{10}\right) & =1-\sum_{k=1}^{10 \log N^{\prime}} \mathbb{P}(|C(x)|=k)-\sum_{k=10 \log N^{\prime}}^{N^{\prime} / 10} \mathbb{P}(|C(x)|=k) \\
& \geq 1-\exp \left[200 \log ^{2} N^{\prime} / N^{\prime}\right] \sum_{k=1}^{10 \log N^{\prime}} \frac{k^{k-1}}{k !} \gamma^{k-1} e^{-k \gamma}-\sum_{k=10 \log N^{\prime}}^{N^{\prime} / 10} \frac{k^{k-1} e^{-k}}{k !} \frac{1}{2} e^{-k / 10} \\
& >1-\exp \left[200 \log ^{2} N^{\prime} / N^{\prime}\right] \sum_{k=1}^{\infty} \frac{k^{k-1}}{k !} \gamma^{k-1} e^{-k \gamma}-\frac{1}{2 N^{\prime}} \\
& =1-\exp \left[200 \log ^{2} N^{\prime} / N^{\prime}\right](1-\vartheta(\gamma-1))-\frac{1}{2 N^{\prime}} \\
& =\vartheta(\gamma-1)-O\left(\log ^{2} N^{\prime} / N^{\prime}\right) .
\end{aligned}
$$

Thus with probability $(1-o(1)) \vartheta(\gamma-1)^{2}>0.63$ (for large enough $N^{\prime}$ ), both the in-graph and out-graph have size at least $N^{\prime} / 10$.

Therefore, if we try $N^{\prime} / 50$ candidates, the probability that we lose too many variables on failed candidates, or have enough variables but still fail to find a vertex with in-graph and out-graph of size at least $N^{\prime} / 10$, is bounded by

$$
\frac{3^{N^{\prime} / 50}}{e^{N^{\prime} / 20}}+0.37^{N^{\prime} / 50}
$$

which establishes the lemma.

\section{Appendix A. Relation Between $F_{n, p}$ And $F_{n, m}$}

While the literature focuses on $F_{n, m}$, where a given number of clauses are specified, most of our theorems and proofs are done for $F_{n, p}$, where each clause has some independent chance of appearing in the formula, and the total number of clauses is random. In this appendix, we discuss the relation between the models $F_{n, m}$ and $F_{n, p}$. With respect to monotone properties, these two models are practically interchangeable, provided $m$ is about $4\left(\begin{array}{l}n \\ 2\end{array}\right) p$, the expected number of clauses in $F_{n, p}$. Write $N$ for the number of 2-clauses 
on $x_{1}, \ldots, x_{n}$, so that $N=2 n(n-1)$, and let $M_{N, p}$ be a binomial random variable with parameters $N$ and $p$. Then we have

$$
\begin{aligned}
\mathbb{P}\left(\operatorname{SAT}\left(F_{n, p}\right)\right) & =\sum_{m=0}^{N} \mathbb{P}\left(M_{N, p}=m\right) \mathbb{P}\left(\operatorname{SAT}\left(F_{n, m}\right)\right), \\
E\left(S\left(F_{n, p}\right)\right) & =\sum_{m=0}^{N} \mathbb{P}\left(M_{N, p}=m\right) E\left(S\left(F_{n, m}\right)\right)
\end{aligned}
$$

and

$$
E\left(S^{2}\left(F_{n, p}\right)\right)=\sum_{m=0}^{N} \mathbb{P}\left(M_{N, p}=m\right) E\left(S^{2}\left(F_{n, m}\right)\right) .
$$

Since $\mathbb{P}\left(\operatorname{SAT}\left(F_{n, m}\right)\right)$ is a monotone decreasing function of $m$, for every $0<m<N$ we have

$$
\mathbb{P}\left(\operatorname{SAT}\left(F_{n, m}\right)\right)-\mathbb{P}\left(M_{N, p}>m\right) \leq \mathbb{P}\left(\operatorname{SAT}\left(F_{n, p}\right)\right) \leq \mathbb{P}\left(\operatorname{SAT}\left(F_{n, m}\right)\right)+\mathbb{P}\left(M_{N, p}<m\right) .
$$

Similarly, we have

$$
\begin{aligned}
& \mathbb{P}\left(\operatorname{UNSAT}\left(F_{n, m}\right)\right)- \mathbb{P}\left(M_{N, p}<m\right) \\
& \leq \mathbb{P}\left(\operatorname{UNSAT}\left(F_{n, p}\right)\right) \\
& \leq \mathbb{P}\left(\operatorname{UNSAT}\left(F_{n, m}\right)\right)+\mathbb{P}\left(M_{N, p}>m\right), \\
& E\left(S\left(F_{n, m}\right)\right)-2 n \mathbb{P}\left(M_{N, p}<m\right) \leq E\left(S\left(F_{n, p}\right)\right) \leq E\left(S\left(F_{n, m}\right)\right)+2 n \mathbb{P}\left(M_{N, p}>m\right),
\end{aligned}
$$

and

$$
E\left(S^{2}\left(F_{n, m}\right)\right)-4 n^{2} \mathbb{P}\left(M_{N, p}<m\right) \leq E\left(S^{2}\left(F_{n, p}\right)\right) \leq E\left(S^{2}\left(F_{n, m}\right)\right)+4 n^{2} \mathbb{P}\left(M_{N, p}>m\right) .
$$

In bounding the probability in the tail of the binomial distribution, we shall make use of the following Chernoff type inequality (see e.g. [McD89]):

$$
\mathbb{P}\left(\left|M_{N, p}-p N\right| \geq \rho p N\right) \leq e^{-\rho^{2} p N / 3},
$$

provided $0<p \leq 1 / 2$.

To bound the probability of unsatisfiability in the subcritical regime, the expected size of the spine, and the second moment of the size of the spine, we set

$$
p=\frac{1+\lambda n^{-1 / 3}}{2 n}
$$

and

with

$$
m=\left(1+\lambda^{\prime} n^{-1 / 3}\right) n,
$$

$$
\lambda^{\prime}=\lambda \pm n^{-1 / 12} .
$$


Then the probability that $M_{N, p}$ is too large or too small (compared with $m$ ) is

$$
\exp \left(-\Theta\left(n^{1 / 6}\right)\right)=o\left(1 / n^{2}\right)
$$

From this we see that our bounds for $F_{n, p}$ imply the desired bounds for $F_{n, m}$ in the subcritical regime.

To convert the bounds on the probability of satisfiability on the right from $F_{n, p}$ to $F_{n, m}$, because this probability is so small, in order for it to dwarf $\mathbb{P}\left(M_{N, p}<m\right)$ and $\mathbb{P}\left(M_{N, p}>m\right)$, we need to have a larger gap between $\lambda$ and $\lambda^{\prime}$. We set

$$
\lambda^{\prime}=\lambda \pm \frac{\lambda^{6 / 5}}{n^{1 / 15}}
$$

Then the probability of a large deviation is at most

$$
\exp \left[-\Theta\left(\lambda^{12 / 5} n^{1 / 5}\right)\right]=o\left(\exp \left[-\Theta\left(\lambda^{3}\right)\right]\right)
$$

if $\lambda$ is small enough compared to $n^{1 / 3}$. Furthermore, $\lambda^{\prime} / \lambda$ is arbitrarily close to 1 provided $\lambda$ is sufficiently small compared to $n^{1 / 3}$. Thus our bounds for satisfiability of $F_{n, p}$ in the supercritical regime carry over to corresponding bounds for $F_{n, m}$.

\section{Appendix B. Proof of Lemma 5.5}

In this appendix, we prove Lemma 5.5. We start with the proof of statement (i). To this end, we again use that the cluster size distribution in $G_{n, \widetilde{p}}, \widetilde{p}=2 p-p^{2}$, is stochastically dominated by a Poisson birth process with parameter

$$
\hat{\kappa}=n \log 1 /(1-\widetilde{p})=2 n \log 1 /(1-p)=2 n p(1+O(1 / n)) .
$$

Writing this parameter as $\hat{\kappa}=1+\hat{\varepsilon}$, and observing that the probability that a Poisson birth tree with parameter $\hat{\kappa}$ has size $k$ is $(1 / \hat{\kappa})\left(k^{k-1} / k !\right)\left(\hat{\kappa} e^{-\hat{\kappa}}\right)^{k}$, we therefore get

$$
\begin{aligned}
\sum_{k \geq n^{2 / 3} / \lambda_{n}} Q_{n, p}(k) & \leq \vartheta(\hat{\varepsilon})+\frac{1}{\hat{\kappa}} \sum_{k \geq n^{2 / 3} / \lambda_{n}} \frac{k^{k-1}}{k !}\left(\hat{\kappa} e^{-\hat{\kappa}}\right)^{k} \\
& \leq \vartheta(\hat{\varepsilon})+\frac{1}{\hat{\kappa}} \sum_{k \geq n^{2 / 3} / \lambda_{n}} \frac{1}{\sqrt{2 \pi k^{3}}}\left(\hat{\kappa} e^{1-\hat{\kappa}}\right)^{k}
\end{aligned}
$$

where

$$
\vartheta(\hat{\varepsilon})=1-\frac{1}{\hat{\kappa}} \sum_{k=0}^{\infty} \frac{k^{k-1}}{k !}\left(\hat{\kappa} e^{-\hat{\kappa}}\right)^{k}
$$

is the survival probability in a Poisson birth process with parameter $\hat{\kappa}$. If $\varepsilon \leq \varepsilon_{0}$, then

$$
\hat{\kappa} e^{1-\hat{\kappa}} \leq e^{-c \varepsilon^{2}}
$$

for some constant $c=c\left(\varepsilon_{0}\right)$, so that

$$
\sum_{k \geq n^{2 / 3} / \lambda_{n}} Q_{n, p}(k) \leq \vartheta(\hat{\varepsilon})+O\left(\frac{1}{\sqrt{\lambda_{n} n^{2 / 3}}} e^{-c \lambda_{n}}\right) \leq \vartheta(\hat{\varepsilon})\left(1+O\left(e^{-c \lambda_{n}}\right)\right)
$$


where we have used that $\vartheta(\hat{\varepsilon})=\Theta(\hat{\varepsilon})=\Theta\left(\lambda_{n} n^{-1 / 3}\right)$ in the last step. Since $\hat{\varepsilon}=\varepsilon+$ $O\left(n^{-1}\right)=\varepsilon\left(1+O\left(\lambda_{n}^{-3}\right)\right)$, we conclude that

$$
\begin{aligned}
\sum_{k \geq n^{2 / 3} / \lambda_{n}} Q_{n, p}(k) & \leq \vartheta(\varepsilon)\left(1+O\left(\lambda_{n}^{-3}\right)\right) \\
& =\Theta\left(\lambda_{n} n^{-1 / 3}\right) .
\end{aligned}
$$

To prove a lower bound, we show that

$$
\sum_{k<n^{2 / 3} / \lambda_{n}} Q_{n, p}(k) \leq 1-\vartheta(\varepsilon)\left(1+O\left(\lambda_{n}^{-2}\right)\right) .
$$

Together with (ii), which will be proved below, this gives the desired bound. To prove (B.8), we will first show that for $k \leq n^{2 / 3} / \lambda_{n}$,

$$
Q_{n, p}(k) \leq \frac{1}{1+\varepsilon^{\prime}} \frac{k^{k-1}}{k !}\left(\left(1+\varepsilon^{\prime}\right) e^{-\left(1+\varepsilon^{\prime}\right)}\right)^{k}\left[1+O\left(\frac{k^{3 / 2}}{n}\right)\right],
$$

where $\varepsilon^{\prime}$ is defined as the positive solution of

$$
\left(1+\varepsilon^{\prime}\right) e^{-\varepsilon^{\prime}}=(1+\varepsilon) e^{-\varepsilon} e^{\varepsilon^{2} \lambda^{-2} / 2} .
$$

Indeed, using (5.13), we bound

$$
\begin{aligned}
Q_{n, p}(k) & \leq \frac{1}{n}\left(\begin{array}{l}
n \\
k
\end{array}\right)(2 p k)^{k-1} e^{-p\left(2 n k-k^{2}-3 k+2\right)} S_{2 p-p^{2}}(k) \\
& =\frac{(2 p n k)^{k-1}}{k !}\left[\prod_{i=0}^{k-1}\left(1-\frac{i}{n}\right)\right] e^{-p\left(2 n k-k^{2}-3 k+2\right)} S_{2 p-p^{2}}(k) \\
& \leq \frac{(2 p n k)^{k-1}}{k !} e^{-k(k-1) / 2 n} e^{-p\left(2 n k-k^{2}-3 k+2\right)} S_{2 p-p^{2}}(k) .
\end{aligned}
$$

For $k \leq n^{2 / 3} / \lambda_{n}$, we have $S_{2 p-p^{2}}(k)=1+O\left(k^{3 / 2} / n\right)$. Combined with the observation that $p k^{2}-k^{2} / 2 n=\varepsilon k^{2} / 2 n \leq k \varepsilon^{2} / 2 \lambda^{2}$ we therefore get

$$
\begin{aligned}
Q_{n, p}(k) & \leq \frac{(2 p n k)^{k-1}}{k !} e^{-\left(2 p n-\varepsilon^{2} \lambda^{-2} / 2\right) k}\left[1+O\left(\frac{k^{3 / 2}}{n}\right)\right] \\
& =\frac{1}{1+\varepsilon} \frac{k^{k-1}}{k !}\left((1+\varepsilon) e^{-\left(1+\varepsilon-\varepsilon^{2} \lambda^{-2} / 2\right)}\right)^{k}\left[1+O\left(\frac{k^{3 / 2}}{n}\right)\right] .
\end{aligned}
$$

Using the definition $(\mathbb{B . 1 0})$ of $\varepsilon^{\prime}$ and observing that $\varepsilon^{\prime} \leq \varepsilon$, we get $(\mathbb{B . 9})$.

As a consequence of $(\overline{B .9})$, we now have

$$
\begin{aligned}
\sum_{k<n^{2 / 3} / \lambda_{n}} Q_{n, p}(k) & \leq \sum_{k<n^{2 / 3} / \lambda_{n}} \frac{1}{1+\varepsilon^{\prime}} \frac{k^{k-1}}{k !}\left(\left(1+\varepsilon^{\prime}\right) e^{-\left(1+\varepsilon^{\prime}\right)}\right)^{k}\left[1+O\left(\frac{k^{3 / 2}}{n}\right)\right], \\
& \leq 1-\vartheta\left(\varepsilon^{\prime}\right)+O\left(\sum_{k<n^{2 / 3} / \lambda_{n}} \frac{k^{3 / 2}}{n} \frac{1}{1+\varepsilon^{\prime}} \frac{k^{k-1}}{k !}\left(\left(1+\varepsilon^{\prime}\right) e^{-\left(1+\varepsilon^{\prime}\right)}\right)^{k}\right) .
\end{aligned}
$$


Observing that $\varepsilon \leq \varepsilon_{0}$ implies $\varepsilon^{\prime} \leq \varepsilon_{0}$, which implies a bound of the form (B.4) for $\kappa^{\prime}=1+\varepsilon^{\prime}$, we now bound the sum over $k$ as follows:

$$
\begin{gathered}
\sum_{k<n^{2 / 3} / \lambda_{n}} \frac{k^{3 / 2}}{n} \frac{1}{\kappa^{\prime}} \frac{k^{k-1}}{k !}\left(\kappa^{\prime} e^{-\kappa^{\prime}}\right)^{k} \leq \sum_{k<n^{2 / 3} / \lambda_{n}} \frac{k^{3 / 2}}{n} \frac{1}{\sqrt{2 \pi k^{3}}}\left(\kappa^{\prime} e^{1-\kappa^{\prime}}\right)^{k} \\
=O\left(\frac{1}{n}\right) \sum_{k<n^{2 / 3} / \lambda_{n}} e^{-c \varepsilon^{2} k}=O\left(\frac{1}{n}\right) \frac{1}{1-e^{-c \varepsilon^{2}}} \\
=O\left(\frac{1}{n \varepsilon^{2}}\right)=\vartheta(\varepsilon) O\left(\lambda_{n}^{-3}\right) .
\end{gathered}
$$

Together with the observation that $\varepsilon^{\prime}=\varepsilon\left(1-\Theta\left(\lambda_{n}^{-2}\right)\right)$ for $\varepsilon$ small enough, the bounds (B.14) and (B.15) imply (B.8).

Next we prove statement (ii). To this end, we use a refinement (due to Alon and Spencer [AS92]) of the viewpoint employed by Karp [Kar90 and used here in the proof of Lemma 5.1. Define $N_{0}=n-1$, and for positive $t, N_{t}=\operatorname{Binomial}\left(N_{t-1}, 1-\widetilde{p}\right)$. Then let $Y_{t}=n-t-N_{t}$, and define $T$ to be the least $t$ such that $Y_{t}=0$. This random variable $T$ has the same distribution as the size of the connected component containing a given vertex in $G_{n, \widetilde{p}}$ AS92.

Condition the $N_{t}$ process to be small enough that $Y_{t}$ is positive whenever $t<n^{2 / 3} / \lambda$ (i.e. $T \geq n^{2 / 3} / \lambda$ ). How does this affect the distribution of $N_{t}$ for larger values of $t$ ? We can think of the $N_{t}$ 's as being determined by a collection of i.i.d. 0-1 random variables with probability $1-\widetilde{p}$. Each $N_{t}$ is monotone increasing in these variables. By FKG, the above conditioning can only decrease the distribution of $N_{t}$ (increase the distribution of $Y_{t}$ ) for any given value of $t$, and in particular makes it less likely that $Y_{t} \leq 0$ for some $t$ in a given range. Thus we have

$$
\begin{aligned}
\mathbb{P}\left(n^{2 / 3} / \lambda \leq T \leq n^{2 / 3} \lambda\right) & =\mathbb{P}\left(n^{2 / 3} / \lambda \leq T\right) \mathbb{P}\left(\exists t: Y_{t} \leq 0, n^{2 / 3} / \lambda \leq t \leq \lambda n^{2 / 3} \mid n^{2 / 3} / \lambda \leq T\right) \\
& =O\left(\frac{\lambda}{n^{1 / 3}}\right) \mathbb{P}\left(\exists t: Y_{t} \leq 0, n^{2 / 3} / \lambda \leq t \leq \lambda n^{2 / 3}\right) \quad \text { by (B. } 1 \text { ) } \\
& =O\left(\frac{\lambda}{n^{1 / 3}}\right)\left[\mathbb{P}\left(Y_{n^{2 / 3} / \lambda} \leq 0\right)+\mathbb{P}\left(\exists t: Y_{t}=0, n^{2 / 3} / \lambda \leq t \leq \lambda n^{2 / 3}\right)\right],
\end{aligned}
$$

where in the last line we used $Y_{t+1} \geq Y_{t}-1$.

Let $X_{t}$ denote the event that $Y_{t}=0$. Let $Z_{t}$ denote the event that $Y_{t}=0$ and $Y_{s}>0$ for $n^{2 / 3} / \lambda \leq s<t$. Let

$$
S=\sum_{n^{2 / 3} / \lambda \leq t \leq \lambda n^{2 / 3}+n^{2 / 3} / \lambda^{2}} \mathbb{I}_{X_{t}},
$$

where as before $\mathbb{I}_{A}$ denotes the indicator of the event $A$. We have

$$
S \geq \sum_{n^{2 / 3} / \lambda \leq t \leq \lambda n^{2 / 3}} \mathbb{I}_{Z_{t}} \sum_{0 \leq \Delta \leq n^{2 / 3} / \lambda^{2}} \mathbb{I}_{X_{t+\Delta}},
$$


so that

$$
\begin{aligned}
E[S] & \geq \sum_{n^{2 / 3} / \lambda \leq t \leq \lambda n^{2 / 3}} \mathbb{P}\left[Z_{t}\right] E\left[\sum_{0 \leq \Delta \leq n^{2 / 3} / \lambda^{2}} \mathbb{I}_{X_{t+\Delta}} \mid Z_{t}\right] \\
& =\sum_{n^{2 / 3} / \lambda \leq t \leq \lambda n^{2 / 3}} \mathbb{P}\left[Z_{t}\right] \sum_{0 \leq \Delta \leq n^{2 / 3} / \lambda^{2}} \mathbb{P}\left(Y_{t+\Delta}=0 \mid Y_{t}=0\right) \\
& \geq \sum_{n^{2 / 3} / \lambda \leq t \leq \lambda n^{2 / 3}} \mathbb{P}\left[Z_{t}\right] \min _{t} \sum_{0 \leq \Delta \leq n^{2 / 3} / \lambda^{2}} \mathbb{P}\left(Y_{t+\Delta}=0 \mid Y_{t}=0\right) \\
& \geq \mathbb{P}\left[\exists t: Y_{t}=0\right] \min _{t} \sum_{0 \leq \Delta \leq n^{2 / 3} / \lambda^{2}} \mathbb{P}\left(Y_{t+\Delta}=0 \mid Y_{t}=0\right),
\end{aligned}
$$

where in $(\mathbb{B} .17)$ we used the fact that the $Y_{t}$ 's are Markovian, and in the last line the range of $t$ is given by $n^{2 / 3} / \lambda \leq t \leq \lambda n^{2 / 3}$.

Next we estimate $\mathbb{P}\left[Y_{t+\Delta}=0 \mid Y_{t}=0\right]$ and $\mathbb{P}\left[Y_{t}=0 \mid Y_{0}=1\right]$. Since

$$
Y_{s+\Delta}=Y_{s}-\Delta+\operatorname{Binomial}\left(n-s-Y_{s}, 1-(1-\widetilde{p})^{\Delta}\right),
$$

we seek

$$
\mathbb{P}(\operatorname{Binomial}(m, r)=E[\operatorname{Binomial}(m, r)]+x)
$$

where $m=n-s-Y_{s}, r=1-(1-\widetilde{p})^{\Delta}$, and $x=\Delta-Y_{s}-m r$.

We have $m=(1+o(1)) n$ and $r=\Delta \widetilde{p}(1+O(\Delta \widetilde{p}))=\Theta(\Delta \widetilde{p})$. Thus $E[\operatorname{Binomial}(m, r)]=$ $m r=\Theta(\Delta)$ and $\operatorname{Var}[\operatorname{Binomial}(m, r)]=\Theta(\Delta)$. We approximate $x$ by

$$
\begin{aligned}
x & =\Delta-Y_{s}-\left(n-s-Y_{s}\right)\left(\Delta \widetilde{p}+O\left(\Delta^{2} \widetilde{p}^{2}\right)\right) \\
& =-Y_{s}+\Delta\left(1-\widetilde{p} n+\widetilde{p} s+\widetilde{p} Y_{s}+O\left(\Delta n \widetilde{p}^{2}\right)\right) .
\end{aligned}
$$

Now assuming $Y_{s}=0$ or 1 , and $s=O\left(\lambda n^{2 / 3}\right)$, and writing $\widetilde{p}$ as $(1+\widetilde{\varepsilon}) / n$, we further approximate

$$
\begin{aligned}
x & =-Y_{s}+\Delta\left(-\widetilde{\varepsilon}+O\left(\lambda n^{-1 / 3}\right)+O(1 / n)+O\left(\lambda n^{-1 / 3}\right)\right) \\
& =-Y_{s}+\Delta O(\varepsilon) \\
& =\Delta O(\varepsilon) \text { if } Y_{s}=0 .
\end{aligned}
$$

The normal approximation to the binomial is valid when

$$
x \ll \operatorname{Var}[\operatorname{Binomial}(m, r)]^{2 / 3} ;
$$

see Feller [Fel68, Volume I, chapter VII.3]. In particular, if $x=O\left(\operatorname{Var}[\operatorname{Binomial}(m, r)]^{1 / 2}\right)=$ $O\left(\Delta^{1 / 2}\right)$, which happens when $\Delta=O\left(1 / \varepsilon^{2}\right)=O\left(n^{2 / 3} / \lambda^{2}\right)$, we have

$$
\mathbb{P}[\operatorname{Binomial}(m, r)=E[\operatorname{Binomial}(m, r)]+x]=\Theta\left(\operatorname{Var}[\operatorname{Binomial}(m, r)]^{-1 / 2}\right)=\Theta\left(\Delta^{-1 / 2}\right)
$$


which implies

$$
\mathbb{P}\left(Y_{s+\Delta}=0 \mid Y_{s}=0\right)=\Theta\left(\Delta^{-1 / 2}\right)
$$

and hence

$$
\sum_{0 \leq \Delta \leq n^{2 / 3} / \lambda^{2}} \mathbb{P}\left(Y_{t+\Delta}=0 \mid Y_{t}=0\right)=\left.\Theta\left(\Delta^{1 / 2}\right)\right|_{0} ^{n^{2 / 3} / \lambda^{2}}=\Theta\left(n^{1 / 3} / \lambda\right) .
$$

Next we estimate $E[S]$. If $s=0$ and $t=\Delta$, we bound $x$ by

$$
\begin{aligned}
x & =t-1-(n-1)\left(1-(1-\widetilde{p})^{t}\right) \leq t-n+n(1-\widetilde{p})^{t} \leq t-n t \widetilde{p}+n\left(\begin{array}{l}
t \\
2
\end{array}\right) \widetilde{p}^{2} \\
& \leq t-n t\left(2 p-p^{2}\right)+2 n t(t-1) p^{2} \leq t-n t 2 p+2 n t^{2} p^{2} \leq-t \varepsilon+t^{2}(1+\varepsilon)^{2} /(2 n) \\
& \leq-t \varepsilon / 9,
\end{aligned}
$$

provided $t \leq\left(\lambda+1 / \lambda^{2}\right) n^{2 / 3} \leq n$ and $\varepsilon<1 / 3$. The deviations $x$ from the mean are too large for the normal approximation to be valid. In this case one would typically use the Chernoff bound, but we also need the $1 / \sqrt{\operatorname{Var}}$ term not found in the standard Chernoff bound. Thus we note that the following variation of the bound is easily deduced from the derivation given by Feller of the normal approximation to the binomial distribution.

$$
\begin{aligned}
\mathbb{P}\left[Y_{t}=0\right] & =O\left(\frac{1}{\sqrt{t}} e^{-\Theta\left(x^{2} / t\right)}\right) \\
& =O\left(\frac{1}{\sqrt{t}} e^{-\Theta\left(t \varepsilon^{2}\right)}\right) \quad \text { recalling }|x| \geq t \varepsilon / 9 \\
& =O\left(\frac{1}{\sqrt{n^{2 / 3} / \lambda}} e^{-\Theta(\lambda)}\right) \quad \text { since } t \geq n^{2 / 3} / \lambda \\
& =O\left(n^{-1 / 3} e^{-\Theta(\lambda)}\right) .
\end{aligned}
$$

Now summing over $t$, we get

$$
E[S]=\sum_{n^{2 / 3} / \lambda \leq t \leq \lambda n^{2 / 3}+n^{2 / 3} / \lambda^{2}} \mathbb{P}\left[Y_{t}=0\right]=O\left(n^{1 / 3} e^{-\Theta(\lambda)}\right) .
$$

Combining the bounds $(\overline{B .18}),(\overline{B .19})$, and $(\overline{B .21})$, we see that

$$
\mathbb{P}\left[Y_{t}=0 \text { for some } t \text { such that } n^{2 / 3} / \lambda \leq t \leq \lambda n^{2 / 3}\right]=O(\exp (-\Theta(\lambda))) .
$$

We also need

$$
\mathbb{P}\left[Y_{n^{2 / 3} / \lambda} \leq 0\right] \leq \exp (-\Theta(\lambda))
$$

which follows from the straight Chernoff bound. Substituting (B.22) and (B.23) into (B.16) we obtain the desired inequality

$$
\mathbb{P}\left[n^{2 / 3} / \lambda \leq|C(0)| \leq \lambda n^{2 / 3}\right]=O\left(n^{-1 / 3} \exp (-\Theta(\lambda))\right) .
$$


Acknowledgements: The authors thank Dimitris Achlioptas and Riccardo Zecchina for many useful discussions; in particular, it was Riccardo Zecchina who suggested to us the significance of the backbone density. We also thank the Institute for Advanced Study in Princeton, where this work was begun. Finally, one of us (B. Bollobás) thanks Microsoft Research, where most of this work was done.

\section{REFERENCES}

Ach00. D. Achlioptas. Setting 2 variables at a time yields a new lower bound for random 3-SAT (extended abstract), Proc. 32nd ACM Symposium on Theory of Computing, 28-37 (2000).

Ald90. D.J. Aldous. A random walk construction of uniform spanning trees and uniform labelled trees, SIAM J. on Discrete Mathematics, 3(4):450-465 (1990).

AM98. D. Achlioptas and M. Molloy. Personal communication (1998).

APT79. B. Aspvall, M.F. Plass and R.E. Tarjan. A linear-time algorithm for testing the truth of certain quantified Boolean formulas, Inf. Process. Lett. 8:121-123 (1979).

AS92. $\quad$ N. Alon and J. Spencer. The Probabilistic Method, John Wiley \& Sons, xiii + 254 pp (1992).

AS00. D. Achlioptas and G.B. Sorkin. Optimal myopic algorithms for random 3-SAT, Proc. 41st Symposium on the Foundations of Computer Science, 590-600 (2000).

BBCK98. B. Bollobás, C. Borgs, J.T. Chayes and J.H. Kim. Lecture at the Workshop on the Interface between Statistical Physics and Computer Science, Torino, Italy, unpublished (1998).

BBCKW00. B. Bollobás, C. Borgs, J.T. Chayes, J.H. Kim and D.B. Wilson. Critical exponents of the 2-SAT transition, in preparation (2000).

BCKS98a. C. Borgs, J.T. Chayes, H. Kesten and J. Spencer. Uniform boundedness of critical crossing probabilities implies hyperscaling, to appear in Rand. Struc. Alg.

BCKS98b. C. Borgs, J.T. Chayes, H. Kesten and J. Spencer. Birth of the infinite cluster: Finite-size scaling in percolation, preprint (1998).

BCM90. $\quad$ E.A. Bender, E.R. Canfield and B.D. McKay. The asymptotic number of labeled connected graphs with a given number of vertices and edges, Rand. Struc. Alg. 1:127-169 (1990).

BFU93. $\quad$ A. Broder, A. Frieze and E. Upfal. On the satisfiability and maximum satisfiablity of random 3-CNF formulas, Proc. 4th ACM-SIAM Symposium on Discrete Algorithms, 322-330 (1993).

Bol84. $\quad$ B. Bollobás. The evolution of random graphs, Trans. Amer. Math. Soc. 286:257-274 (1984).

Bol85. $\quad$ B. Bollobás. Random Graphs, Academic Press, London, xvi + 447 pp (1985).

Bri89. V.E. Britikov. O strukture sluchaŭnogo grafa vblizi kriticheskoŭ tochki, Diskretnẫa Matematika, 1:121-128 (1989). English translation, On the random graph structure near the critical point, Discrete Math. Appl., 1:301-309 (1991).

CA93. J.M. Crawford and L.D. Auton. Experimental results on the crossover point in satisfiability problems, Proc. 11th Natl. Conf. on Artificial Intelligence, 21-27 (1993).

CF86. M.T. Chao and J. Franco. Probabilistic analysis of two heuristics for the 3-satisfiability problem, SIAM J. on Computing 5:1106-1118 (1986).

CF90. M.T. Chao and J. Franco. Probabilistic analysis of a generalization of the unit-clause literal selection heuristics for the $k$ satisfiable problem, Information Science 51:289-314 (1990).

Cha98. J.T. Chayes. Finite-size scaling in percolation, Proc. of the International Congress of Mathematicians Vol. III (Berlin, 1998), Doc. Math. Extra Vol. III, 113-122 (1998).

Che52. H. Chernoff. A measure of asymptotic efficiency for tests of a hypothesis based on the sum of observations, Ann. Math. Stat. 23:493-507 (1952).

Coo71. S.A. Cook. The complexity of theorem-proving procedures, Proc. 3rd ACM Symposium on Theory of Computing, 151-158 (1971). 
CPS99. J.T. Chayes, A. Puha and T. Sweet. Independent and dependent percolation, Probability theory and applications (Princeton, NJ, 1996), 49-166 in IAS/Park City Math. Ser. Vol. 6, Amer. Math. Soc., Providence, RI (1999).

CR92. V. Chvátal and B. Reed. Mick gets some (the odds are on his side), Proc. 33rd Symposium on the Foundations of Computer Science, 620-627 (1992).

CS88. V. Chvátal and E. Szemerédi. Many hard examples for resolution, J. ACM 35:759-768 (1988).

DB97. O. Dubois and Y. Boufkhad. A general upper bound for the satisfiablity threshold of random $k$-SAT formulas, J. Algorithms 24:395-420 (1997).

DBM99. O. Dubois, Y. Boufkhad, and J. Mandler. Typical random 3-SAT formulae and the satisfiability threshold. Research announcement at ICTP, Sept. 1999. Two-page abstract appears in Proc. 11th ACM-SIAM Symposium on Discrete Algorithms, 126-127 (2000).

Dub91. O. Dubois. Counting the number of solutions for instances of satisfiability, Theoretical Computer Science 81:49-64 (1991).

EF95. A. El Maftouhi and W. Fernandez de la Vega. On random 3-sat. Combin. Probab. Comput. 4:189-195 (1995).

ER60. P. Erdős and A. Rényi. On the evolution of random graphs, Magyar Tud. Akad. Mat. Kutató Int. Közl. 5:17-61 (1960).

ER61. P. Erdős and A. Rényi. On the evolution of random graphs, Bull. Inst. Internat. Statist. 38:343-347 (1961).

FB99. E. Friedgut, with appendix by J. Bourgain. Sharp thresholds of graph properties, and the $k$-sat problem, J. Amer. Math. Soc. 12:1017-1054 (1999).

Fel68. W. Feller. An Introduction to Probability Theory and Its Applications, Volume I, 3rd Edition, John Wiley \& Sons, London, xviii + 509 pp (1968).

Fer92. W. Fernandez de la Vega. On random 2-SAT, unpublished manuscript (1992).

Fer98. W. Fernandez de la Vega. On random 2-SAT (revised version), preprint (1998).

FKG71. C.M. Fortuin, P.W. Kasteleyn and J. Ginibre. Correlation inequalities on some partially ordered sets, Commun. Math. Phys. 22:89-103 (1971).

FP83. J. Franco and M. Paul. Probabilistic analysis of the Davis-Putnam procedure for solving the satisfiability problem, Discrete Applied Mathematics 5:77-87 (1983).

FS96. A. Frieze and S. Suen. Analysis of two simple heuristics for a random instance of $K$-SAT, J. Algorithms 20:312-335 (1996).

GJ79. M.R. Garey and D.S. Johnson. Computers and Intractability: A Guide to the Theory of NP-Completeness, New York, (1979).

GJS76. M.R. Garey, D.S. Johnson and L. Stockmeyer. Some simplified NP-complete graph problems, Theor. Comp. Sci. 1:237-267 (1976).

Goe92. A. Goerdt. A threshold for unsatisfiability, Mathematical Foundations of Computer Science, 17th Intl. Symposium, I.M. Havel and V. Koubek, Eds., Lecture Notes in Computer Science \#629, Springer Verlag, 264-274 (1992).

Goe96. A. Goerdt. A threshold for unsatisfiability, J. Computer and System Sciences 53:469-486 (1996).

Goe99. A. Goerdt. A remark on random 2-SAT, Discrete Applied Mathematics 96-97:107-110 (1999).

Har60. T.E. Harris. A lower bound for the critical probability in certain percolation processes, Proc. Camb. Phil. Soc. 56:13-20 (1960).

Hås97. J. Håstad. Some optimal in-approximability results, Proc. 29th ACM Symposium on Theory of Computation, 1-10 (1997).

JSV00. S. Janson, Y.C. Stamatiou, and M. Vamvakari. Bounding the unsatisfiability threshold of random 3-SAT, Rand. Struc. Alg. 17:103-116 (2000). 
JKŁP94. S. Janson, D. Knuth, T. Łuczak, and B. Pittel. The birth of the giant component, Rand. Struc. Alg. 4:231-358 (1994).

Kar90. R.M. Karp. The transitive closure of a random digraph, Rand. Struc. Alg. 1:73-93 (1990).

KKK96. L. Kirousis, E. Kranakis and D. Krizanc. Approximating the unsatisfiability threshold of random formulas, Proc. 4th European Symposium on Algorithms, 27-38 (1996).

Kle66. D.J. Kleitman. Families of non-disjoint subsets, J. Combinatorial Theory 1:153-155 (1966).

KMPS95. A. Kamath, R. Motwani, K. Palem and P. Spirakis. Tail bounds for occupancy and the satisfiability threshold conjecture, Rand. Struc. Alg. 7:59-89 (1995).

KS94. S. Kirkpatrick and B. Selman. Critical behavior in the satisfiability of random Boolean expressions, Science 264:1297-1301 (1994).

EPW94. T. Łuczak, B. Pittel and J.C. Wierman. The structure of a random graph at the point of the phase trasnsition, Trans. Amer. Math. Soc. 341:721-748 (1994).

LT93. T. Larrabee and Y. Tsuji. Evidence for satisfiability threshold for random 3CNF formulas, Proc. AAAI Symposium on Artificial Intelligence and NP-Hard Problems, 112 (1993).

Łuc90. T. Euczak. Component behavior near the critical point of the random graph process, Rand. Struc. Alg. 1:287-310 (1990).

McD89. C. McDiarmid. On the method of bounded differences. In Surveys in Combinatorics, 1989, pages 148-188 (1989).

MPV87. M. Mézard, G. Parisi and M.A. Virasoro. Spin Glass Theory and Beyond, World Scientific, Singapore (1987).

MSL92. D. Mitchell, B. Selman and H. Levesque. Hard and easy distributions of SAT problems, Proc. 10th Natl. Conf. on Artificial Intelligence, 459-465 (1992).

MZ96. R. Monasson and R. Zecchina. The entropy of the $K$-satisfiability problem, Phys. Rev. Lett. 76:3881 (1996).

MZ97. R. Monasson and R. Zecchina. Statistical mechanics of the random K-SAT model, Phys. Rev. E 56:1357-1370 (1997).

MZKST99. R. Monasson, R. Zecchina, S. Kirkpatrick, B. Selman and L. Troyansky. 2+p-SAT: Relation of typical-case complexity to the nature of the phase transition, Rand. Struc. Alg. 15:414435 (1999).

SK96. B. Selman and S. Kirkpatrick. Critical behavior in the computational cost of satisfiability testing, Artificial Intelligence 81:273-295 (1996).

Ver99. Y. Verhoeven. Random 2-SAT and unsatisfiability, Inf. Process. Lett. 72:119-123 (1999).

Wil98. D.B. Wilson. http://dbwilson.com/2sat-data/ (1998).

Wil00. D.B. Wilson. The empirical values of the critical $k$-SAT exponents are wrong. Preprint math.PR/0005136 (2000).

Wri77. E.M. Wright. The number of connected sparsely edged graphs, J. Graph Theory 1:317-330 (1977).

Wri80. E.M. Wright. The number of connected sparsely edged graphs III. Asymptotic results, $J$. Graph Theory 4:393-407 (1980). 
BÉla BOLlOBÁS

Department of Mathematical Sciences

UNIVERSITY OF MEMPHIS

MEMPhis, TN 38152

And Trinity College

Cambridge CB2 1TQ, England

E-mail address: bollobas@msci.memphis.edu

b.bollobas@dpmms.cam.ac.uk

Christian Borgs

Microsoft RESEARCH

One Microsoft Way

REDMOND, WA 98052

E-mail address: borgs@microsoft.com

Jennifer Tour Chayes

Microsoft Research

One Microsoft WAY

REDMOND, WA 98052

E-mail address: jchayes@microsoft.com

JEONG HAN KIM

Microsoft Research

One Microsoft Way

REDMOND, WA 98052

E-mail address: jehkim@microsoft.com

David Bruce Wilson

Microsoft Research

One Microsoft Way

REDMOND, WA 98052

E-mail address: dbwilson@microsoft.com 